Florida International University FIU Digital Commons

\title{
Cultural Competence in Public Administration: A Framework and Predictors of Cultural Competence for Graduating Masters Students
}

Bridgette E. Cram

bcram002@fiu.edu

DOI: $10.25148 /$ etd.FIDC001935

Follow this and additional works at: https://digitalcommons.fiu.edu/etd

\section{Recommended Citation}

Cram, Bridgette E., "Cultural Competence in Public Administration: A Framework and Predictors of Cultural Competence for Graduating Masters Students" (2017). FIU Electronic Theses and Dissertations. 3459.

https://digitalcommons.fiu.edu/etd/3459 


\section{FLORIDA INTERNATIONAL UNIVERSITY}

Miami, Florida

\section{CULTURAL COMPETENCE IN PUBLIC ADMINISTRATION: A FRAMEWORK AND PREDICTORS OF CULTURAL COMPETENCE FOR GRADUATING MASTERS STUDENTS}

A dissertation submitted in partial fulfillment of the requirements for the degree of DOCTOR OF PHILOSOPHY

in

PUBLIC AFFAIRS

by

Bridgette Cram 
To: Dean John Stack

Green School of International and Public Affairs

This dissertation, written by Bridgette Cram, and entitled Cultural Competence in Public Administration: A Framework and Predictors of Cultural Competence for Graduating Masters Students, having been approved in respect to style and intellectual content, is referred to you for judgment.

We have read this dissertation and recommend that it be approved.

Meredith Newman

Alexander Kroll

Valerie Patterson

Susan P. Himburg

Mohamad Alkadry, Major Professor

Date of Defense: May 30, 2017

The dissertation of Bridgette Cram is approved.

Dean John Stack

Green School of International and Public Affairs

Andrés G. Gil

Vice President for Research and Economic Development and Dean of the University Graduate School

Florida International University, 2017 
(C) Copyright 2017 by Bridgette Cram

All rights reserved. 


\section{DEDICATION}

I dedicate this dissertation to my loved ones. To my mother and father, thank you for demonstrating what hard work and perseverance look like. To my sister, thank you for showing me how important it is to live your passion. To my husband, thank you for your unwavering support and love during this process. And most of all, to my daughter, you are my inspiration. 


\section{ACKNOWLEDGMENTS}

To the members of my dissertation committee, Dr. Meredith Newman, Dr.

Alexander Kroll, Dr. Susan Himburg, and Dr. Valerie Patterson, I thank you for your feedback, guidance, and support throughout this process. To my major professor, Dr. Mohamad Alkadry, thank you for believing in me and helping me to develop my voice.

I also wish to thank Dr. Emel Ganapati for her patience and guidance. I value the research that we were able to work on together.

Thank you to my cohort - Miki, NakHyeok, and Diane - I am so proud of us. And thank you to my Miami family, Diane and Mike, for always being there for me. I am forever grateful to my FIU family. To Susan, Kathy, and Elizabeth, I can't thank you enough for your unending support and encouragement.

Thank you to my parents for ensuring that Nicole and I had every opportunity to succeed. Nicole, thank you for helping me with Ellie so that I could accomplish this goal. Ian, there are not enough words to thank you for everything that you have done. I know that this has not been easy, but you have supported me every step of the way. To Ellie, always remember that anything is possible, I love you. 


\title{
ABSTRACT OF THE DISSERTATION \\ CULTURAL COMPETENCE IN PUBLIC ADMINISTRATION: A FRAMEWORK AND PREDICTORS OF CULTURAL COMPETENCE FOR GRADUATING MASTERS STUDENTS
}

by

\author{
Bridgette Cram
}

Florida International University, 2017

Miami, Florida

Professor Mohamad Alkadry, Major Professor

Cultural competence is a critical aspect of achieving the pillar of social equity in public administration. Cultural competence refers to awareness, knowledge, and skills that support an individual's ability to effectively function in various cultural contexts. Obtaining this set of skills is imperative for serving an increasingly diverse citizenry and workforce. However, several gaps in the literature prevent empirical research on this topic.

Although cultural competence has become increasingly important over the past decade, there lacks a theoretical framework about what influences cultural competence and how to measure cultural competence of public administrators. The purpose of this study was twofold. First, it sought to fill the gaps in the literature through identifying a discipline specific measure of cultural competence, and a set of cultural competencies that public administrators should be able to demonstrate. Second, it was to develop both an indirect and direct measure of cultural competence to test relationships with 
hypothesized predictors: public service motivation, color blind racial attitudes, exposure to diversity, and Lifetime Experience with Discrimination.

To address the primary objective, the results of a Delphi survey of 19 diversity or cultural competence experts in the field were analyzed. The Delphi results served as guidance in creating the dependent variables for this study: The Public Administration Cultural Competence Scale (PACCS) and the Public Administration Cultural Competence Index (PACCI). Fourteen institutions, for a total of 267 student responses, served as the sample to test the relationships with cultural competence and the independent variables.

Results of the analyses demonstrate that the PACCI serves as a preferred assessment of cultural competence, as the PACCS was significantly influenced by social desirability bias. The results also demonstrate evidence for a negative relationship between cultural competence and color blind racial attitudes, and a positive relationship between cultural competence and Lifetime Experience with Discrimination.

Overall, the study provides evidence for the increased need of cultural competence training and integration throughout the curriculum. A commitment to cultural competence integration and assessment is necessary to ensure that future public administrators are prepared to serve an increasingly diverse public. 


\section{TABLE OF CONTENTS}

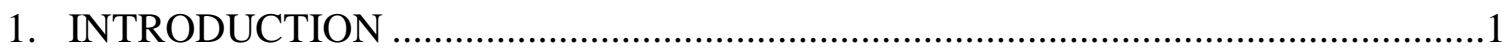

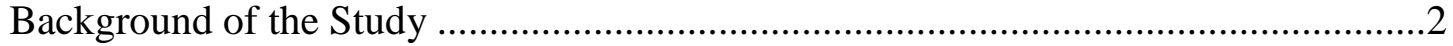

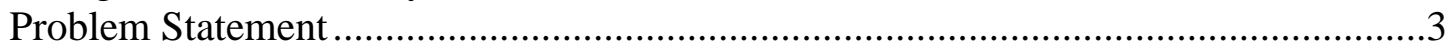

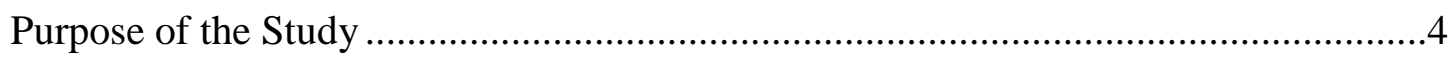

Significance of the Study .......................................................................................

Conceptual Framework ......................................................................................6

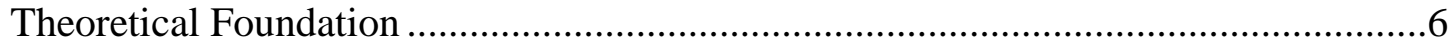

Research Questions and Hypothesis ..........................................................................

Research Design and Methodology …………………..........................................

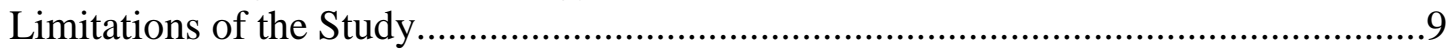

Overview of the Chapters ………………...........................................................

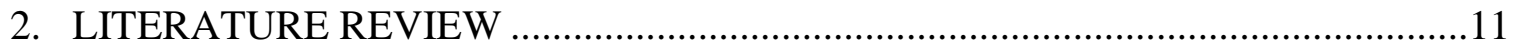

Cultural Competence Defined................................................................................12

Brief History of Cultural Competence .....................................................................13

Development of Cultural Competence in Public Administration ..................................14

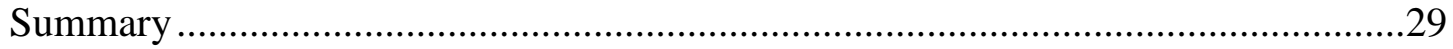

3. CONCEPTUAL FRAMEWORK AND THEORETICAL FOUNDATION .................31

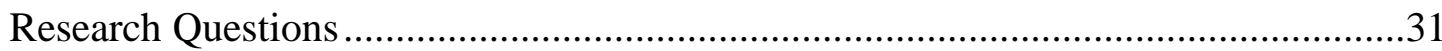

Conceptual Framework and Hypotheses ..................................................................

Theoretical foundation and Hypotheses....................................................................42

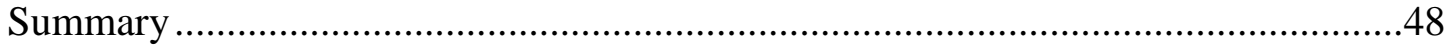

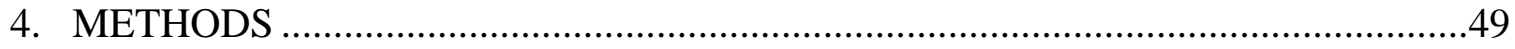

Research Design and Rationale .......................................................................49

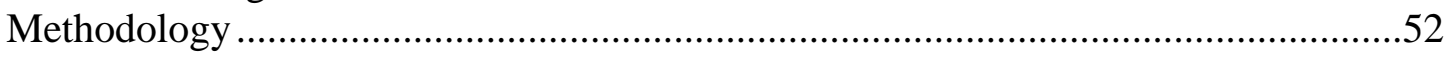

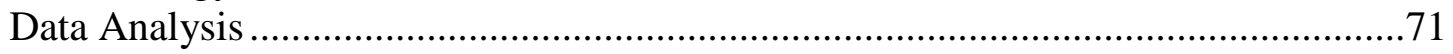

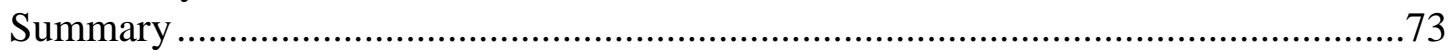

5. MEASUREMENT OF CULTURAL COMPETENCE IN PUBLIC

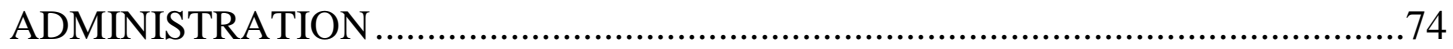

Phase 1A: Modified Delphi method ....................................................................74

Phase 1B: Development of the PACCI and PACCS ………...................................79

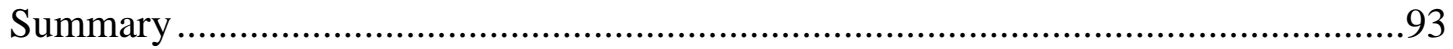

6. PREDICTORS OF CULTURAL COMPETENCE FOR GRADUATING

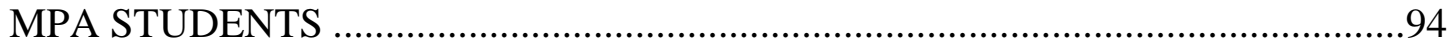

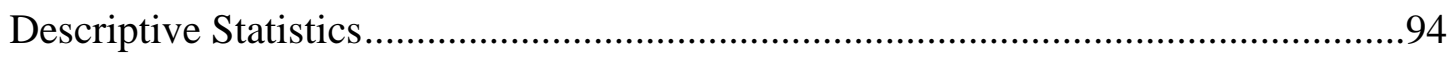

Identifying Predictors of the PACCS ..................................................................98

Identifying Predictors of the PACCI.......................................................................99 
Summary

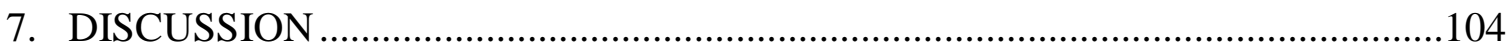

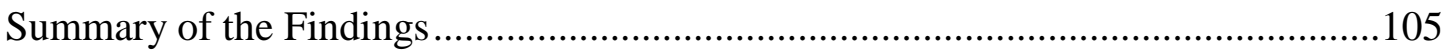

Implications for Public Administration MPA Curriculum ....................................112

Implications for Public Administration Training Programs .................................117

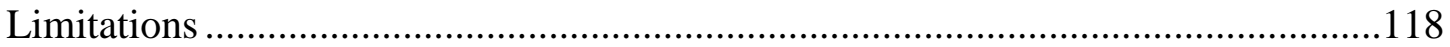

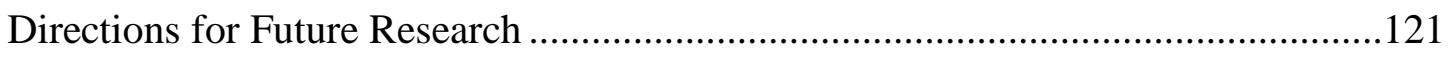

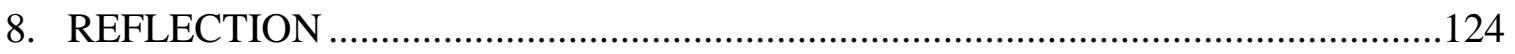

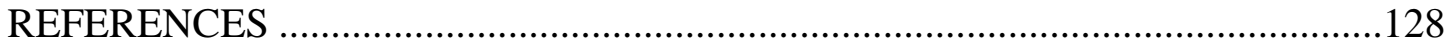

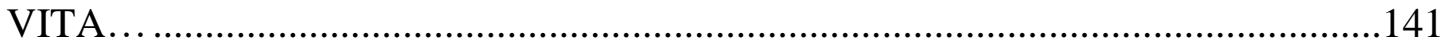




\section{LIST OF TABLES}

TABLE

PAGE

Table 1 Cultural Awareness Components: Conceptual Definitions ............................ 37

Table 2 Cultural Knowledge Components: Conceptual Definitions ........................... 39

Table 3 Cultural Skills Components: Conceptual Definitions................................... 40

Table 4 Ideal Regional Sample Proportion and Actual Regional Sample Proportion .. 55

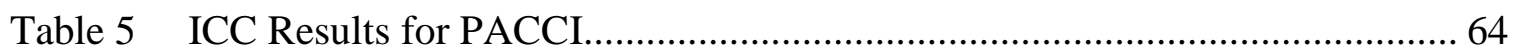

Table 6 Operationalization of Student-Level Control Variables .............................. 70

Table 7 Delphi Results: Cultural Awareness Descriptive Statistics ......................... 76

Table 8 Delphi Results: Cultural Knowledge Descriptive Statistics ......................... 77

Table 9 Delphi Results: Cultural Skills Descriptive Statistics.................................. 78

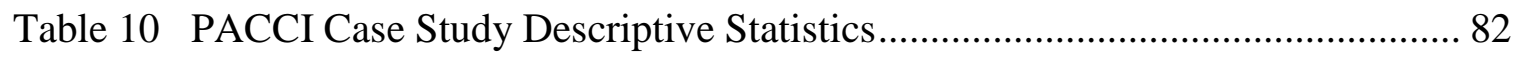

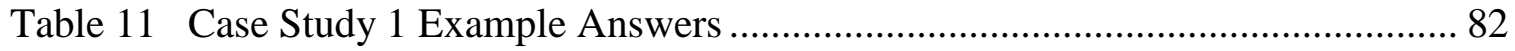

Table 12 Case Study 2 Example Answers ........................................................... 84

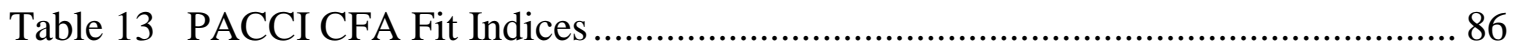

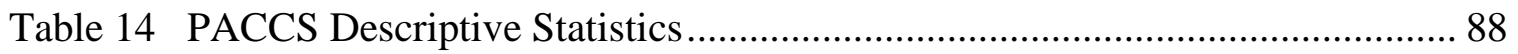

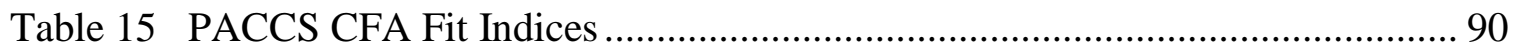

Table 16 Sample Descriptive Statistics for Scale and Ordinal Items .......................... 96

Table 17 Sample Descriptive Statistics for Binary Variables.................................... 96

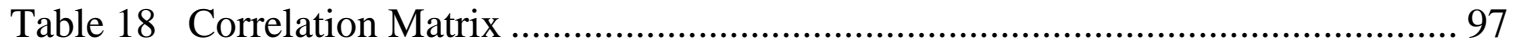

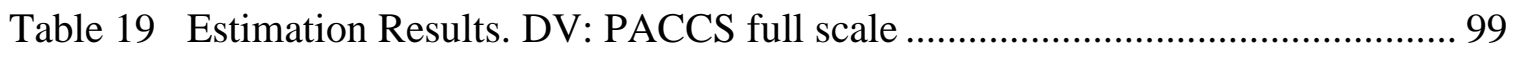

Table 20 Estimation Result. DV: PACCI Case Studies........................................... 101 
Table 21 Estimation Results. DV: PACCI Case Studies; Additional IV: Full PACCS

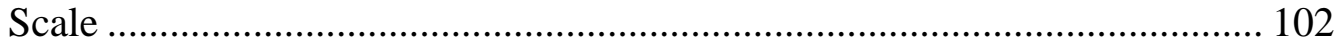




\section{LIST OF FIGURES}

FIGURE

PAGE

Figure 1 Conceptual Model for Cultural Competence. Adapted from Carrizales, 2010;

Cross et al., 1989; Rice \& Mathews, 2012; Tilford Group, 2001 35

Figure 2 Cultural Awareness Components. Adapted from Rice \& Mathews, 2012;

Tilford Group, 2001

Figure 3 Cultural Knowledge Components. Adapted from Carrizales, 2010; Rice \& Mathews, 2012; Tilford Group, 2001 38

Figure 4 Cultural Skills Components. Adapted from Carrizales, 2010; Rice \& Mathews, 2012; Tilford Group, 2001

Figure 5 Hypothesized PACCS Model .................................................................. 42

Figure 6 Predicted Relationship between Predictor Variables and Cultural Competence of Graduating MPA Students ........................................................................ 43

Figure 7 Methodology Used for this Study.......................................................... 51

Figure 8 PACCI Standardized Factor Loadings ................................................. 86

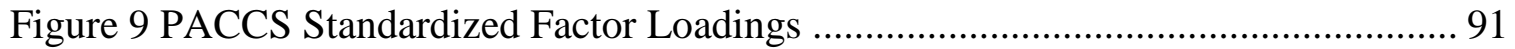




\section{CHAPTER 1}

\section{INTRODUCTION}

Cultural competence encompasses awareness, knowledge, and skills that support an individual's ability to effectively function in various cultural contexts. This has become a critical set of skills for public administrators, due to the increasing demographic diversity of the United States (Carrizales, 2010; Norman-Major \& Gooden, 2012; Rice \& Mathews, 2012; United States Census Bureau, 2014). To effectively serve the public, administrators must have appropriate skills to best interact with citizens and understand how to craft or implement policies to meet their needs. Many public administration faculty and practitioners agree that public administrators should demonstrate cultural competence to work with an increasingly diverse and multicultural society. The field of public administration has not, however, created specific, measurable goals and outcomes for public administration training and education programs (BerryJames, 2012; Norman-Major \& Gooden, 2012).

This lack of unity surrounding cultural competence in public administration contributes to an inability to empirically test the concept, which is necessary so that scholars view it as a serious topic within the field. Therefore, a significant gap exists in creating a case for cultural competence in public administration, due to the lack of empirical research and cultural competence measurement within the discipline.

This study fills these gaps and contributes to the literature on cultural competence within the discipline of public administration; specifically, through the development of a public administration specific definition and set of cultural competencies; creation of direct and indirect measures of cultural competence; and identification of predictors of 
public administrator cultural competence. The implications of the study have both theoretical and practical significance, as it defines cultural competence in the public administration context, determines predictors of cultural competence that can be further empirically tested in other public administration contexts, and gives direction for the design of public administration training and education programs.

\section{Background of the Study}

The concept of cultural competence has existed since the mid 1970's, but did not become a main area of focus until the 1990s (Saha, Beach, \&, 2008; Sue, 2001). The disciplines first concerned with cultural competence were those of psychology, medicine, and nursing (Saha, Beach, \& Cooper, 2008). Interest in cultural competence within these fields grew due to research that demonstrated how culture influences one's view on receiving help. Therefore, to ensure that health professionals deliver services effectively, it became necessary to examine cultural and linguistic barriers and to understand how to best overcome these barriers. In recognition of the importance that cultural and linguistic competence played in health care, the federal government developed standards such as The National Standards for Culturally and Linguistically Appropriate Services in Health and Health Care (CLAS).

The push for social equity that arose in the New Public Administration (NPA) scholarly period prefaced the focus on cultural competence in public administration. NPA signaled a shift away from a sole focus on the three original pillars of public administration: efficiency, effectiveness, and economy. It called for an enhanced advocacy of all citizens, establishing that public administrators worked for the common good of the people. Cultural competence furthers social equity in the practice of public 
administration, as Gooden \& Portillo (2011) note that the role cultural competence plays within the framework of social equity is to compel the public sector to understand and address the needs of the citizens they serve. The main argument for integration of cultural competencies throughout public administration training and education programs is closely tied to this fourth pillar of public administration, social equity.

Given that cultural competence is an important means to promote social equity, and that this promotion of social equity is necessary to "recognize and validate the history and experiences of historically marginalized groups in ways that have been previously unacknowledged" (Lopez-Littleton \& Blessett, 2015, p. 559); how has public administration addressed this need? Scholarly research regarding cultural competence has focused on practical applications in the classroom and for management within public organizations. Although this is important, building a theoretical foundation for cultural competence and showing that cultural competence has empirical significance is of critical importance to the longevity of this topic within the field. This approach has garnered success in the fields of public health, nursing, and other health-related disciplines.

\section{Problem Statement}

Cultural competence encompasses a set of skills that are important for public administrators to have. Determining whether public administrators have these skills and if they can use them is a challenge in both public administration and other disciplines (Berry-James, 2012; Bonilla et al., 2012). This poses a significant problem, as the United States is on track to become a majority-minority nation by 2043 (U.S. Census, 2012). Without a dedication to empirically-driven cultural competence research, it will be difficult for public administration as a field to keep up with these changing demographics 
and address how public service will need to evolve to meet the needs of a more diverse public. While public administration scholars have addressed cultural competence in terms of curriculum integration (Norman-Major, 2012; Bonilla et al., 2012; Norman-Major \& Gooden, 2012), authors of these studies have borrowed from nursing, psychology, business, and public health cultural competence definitions and conceptualizations. Although this interdisciplinary approach helps to contextualize cultural competence, it is critical for public administration scholars to develop a strong theoretical need for research in this area.

\section{Purpose of the Study}

The purpose of this two-phase, sequential mixed-methods study was to define cultural competence in the context of public administration, delineate the cultural competencies public administrators should be able to demonstrate, and identify predictors of cultural competence for future public administrators. The qualitative phase of the study sought to identify public administration specific cultural competencies through a Delphi survey of public administrator professors with expertise in diversity or cultural competence. The findings from the Delphi survey guided development of the Public Administration Cultural Competence Scale (PACCS), an indirect measure of cultural competence; and the Public Administration Cultural Competence Index (PACCI), a direct measure of cultural competence, which were the two dependent variables in the study.

The second phase of the study identified predictors of cultural competence for graduating Master of Public Administration (MPA) students across the nation. The predictor variables in this study were Public Service Motivation (PSM), MPA Exposure to 
Diversity, Colorblind Racial Attitudes (CoBRAS), and Lifetime Experience with Discrimination.

\section{Significance of the Study}

The significance of this study is threefold, as it seeks to advance theory in public administration; inform practice related to cultural competence education, training, and assessment; and fill gaps in the literature related to defining and measuring cultural competence in public administration.

Per the literature, there is a gap in terms of creating a unified conceptual framework for cultural competence in public administration. While scholars have developed a limited number of frameworks, the lack of one agreed upon framework has resulted in the inability to define what cultural competence means for public administration and what cultural competencies public administrators should demonstrate (Rice, 2007b). This study addresses this gap.

Furthermore, as there has only been one empirical study related to the measurement of cultural competence in public administration (Longoria \& Rangajaran, 2015) among MPA students, this study seeks to expand the theory behind the cultural competence of future public administrators through identifying predictors of this set of skills. Thus, the study fills important gaps in the literature through advancing a unified conceptual framework that establishes a definition and set of cultural competencies for public administrators; and advances theory through the identification of predictors of cultural competence for future public administrators. The results of this dissertation serve to improve practice through recommendation for integration of these competencies into MPA programs and workforce training programs. 


\section{Conceptual Framework}

The conceptual framework for this study was guided primarily by Cross' Cultural Competence Model (1989). Cross' model centers on the concept of a cultural competence continuum, which explains the various levels of cultural competence a person or organization possesses. In addition to the continuum, Cross' framework outlines the importance of cultural competence within an organization, and explains how development of competencies should be fostered. The framework also addresses how to adapt service and how to plan for cultural competence. The dissertation pulls from this framework as justification for creation of a discipline specific definition and related competencies. Furthermore, it guides the discussion in Chapter 7, related to how to best integrate these competencies into curriculum and training programs.

The framework developed for this study contains relevant competencies from related disciplines, and previously developed public administration specific frameworks. The derived framework is a three-factor model of cultural competence, comprised of cultural knowledge, cultural awareness, and cultural skills. Chapter 3 details the conceptual framework.

\section{Theoretical Foundation}

While a formal theoretical foundation does not exist for studying cultural competence in public administration, this dissertation begins to build this foundation through examination of PSM, MPA exposure to diversity, CoBRAS, and Lifetime Experience with Discrimination. This study includes PSM due to the link between the proposed motives of public servants, such as compassion and self-sacrifice, with the necessary attitudes and desire that are required of a culturally competent public 
administrator (Perry, 1996; Campinha-Bacote, 2002). MPA Exposure to Diversity represents a combination of theories that state that exposure to diverse classroom experiences and participation in training influences levels of cultural competence (Benkert et al, 2011; Price et al., 2005; Caffrey et al., 2005; Pike \& Kuh, 2006). Neville et al. (2000) developed CoBRAS to measure Colorblind Racial Ideology; studies in other disciplines such as psychology have found that these attitudes are negatively correlated with cultural competence (Neville, Spanierman, \& Doan, 2006; Spanierman, Poteat, Wang, \& Oh, 2008; Chao, Good, Flores, \& Wei, 2010). Lifetime Experience with Discrimination posits that those who experience acts of discrimination are more likely to engage in positive social change (Mattis et al., 2004); this dissertation seeks to examine this relationship in the context of cultural competence. Chapter 3 presents the theoretical foundation.

\section{Research Questions and Hypothesis}

There are two research questions that this dissertation sought to answer. Phase 1 answers the first research question and related sub questions. Phase 2 addresses the second research question. The research questions and related hypotheses are as follows:

1. How is cultural competence measured in public administration?

a. How is cultural competence defined in public administration?

b. What cultural competencies should public administrators be able to demonstrate?

c. What are direct and indirect measures of cultural competence that can be used to measure a public administrator's level of cultural competence? 
H1: Cultural competence consists of three sub-dimensions: cultural awareness, cultural knowledge, and cultural skills.

$\mathrm{H} 2$ : The direct and indirect measures of cultural competence will be positively correlated.

2. What are the predictors of cultural competence for public administrators?

H3: It is hypothesized that PSM is positively related to the PACCS and PACCI scores.

H4: It is hypothesized that MPA Exposure to Diversity is positively related to the PACCS and PACCI scores.

H5: It is hypothesized that CoBRAS is negatively related to the PACCS and PACCI scores.

H6: It is hypothesized that Lifetime Experience with Discrimination is positively related to the PACCS and PACCI scores.

\section{Research Design and Methodology}

This study employs a two-phase mixed methods design. The first phase of the study addresses research question 1 . Phase 1 A consists of a modified Delphi method to develop the cultural competence definition and related competencies. Phase 1B consists of the Confirmatory Factor Analysis (CFA) of both the PACCS and PACCI instruments. Phase 2 employs Ordinary Least Squares (OLS) regression to determine the predictors of cultural competence for graduating MPA students. Data were collected through content analysis and survey instruments and analyzed with NVIVO 10, AMOS23 and STATA14. 


\section{Limitations of the Study}

The study has several limitations. The largest limitation is the generalizability of the survey related to the selected sample of graduating MPA students. While the definition and related competencies developed in this study are generalizable to the discipline of public administration; the results of the regression analyses related to predictors of cultural competence are generalizable to graduating MPA students only.

The second limitation relates to students' perceived exposure to issues related to diversity or cultural competence within their MPA program. Since this question is based on recall, it may not be an accurate reflection of the opportunities offered to students. However, the collection and analyses of program syllabi and a complete documentation of all related events within the MPA program are both time and resource intensive. While future research may benefit from including these additional details, the results are interpreted with this reliance on student perception in mind.

Finally, while the PACCI presents a valid method for directly measuring cultural competence, the confirmatory factor analysis (CFA) is limited to a two-factor solution, as a higher order CFA model could not be produced due to lack of degrees of freedom. However, due to both indirect factors producing similar regression results, this limitation is not detrimental to the discussion and generalizability of results.

\section{Overview of the Chapters}

This dissertation comprises 8 chapters. Chapter 2 presents a review of the literature. This chapter explores the background and development of cultural competence as an area of importance in related disciplines to public administration. Next, it outlines the challenges faced by researchers and practitioners in public administration, and how 
the literature addresses those challenges. It then discusses the development of these skills at both the MPA and public organizational levels; and where gaps in this development exist. Finally, it presents empirical research within public administration

Chapter 3 presents the conceptual framework and theoretical foundation used to guide the study, in addition to the hypotheses related to these frameworks. The chapter presents an overview of Cross' Cultural Competence Model and then describes how the public administration specific conceptual framework was derived. The theoretical foundation portion of the study discusses relevant theories linked to cultural competence in other fields, as well as additional public administration specific theories hypothesized to relate to the cultural competence of graduating MPA students.

Chapter 4 outlines the methodology used to answer the research questions. It presents the research design, participants, instrumentation, data collection, and analysis plan for both phases of the study.

Chapters 5 and 6 present the results of the dissertation. Chapter 5 presents the results of the Delphi method and the CFA results for the PACCS and PACCI instruments. Chapter 6 consists of the results from the regression analyses to determine the predictors of cultural competence for graduating MPA students.

Chapter 7 contextualizes the results and outlines their implications for public administration curriculum and training programs. It concludes with recommendations for future research. Chapter 8 reflects on the findings of the dissertation and concludes the dissertation. 


\section{CHAPTER 2}

\section{LITERATURE REVIEW}

The purpose of this study is to develop a definition and set of cultural competencies, and identify predictors of cultural competence for future public administrators. The ability for public administrators to demonstrate cultural competence is critical, due to the increasing diversity of both citizens and the workforce (Carrizales, 2010; Norman-Major \& Gooden, 2012; Rice \& Mathews, 2012; United States Census Bureau, 2014). While public administration scholars generate literature related to social equity, diversity, and cultural competence, a significant gap exists related to a public administration-specific cultural competence framework. This study proposes to fill this gap through identification of a discipline-specific definition, set of competencies, and predictors for future public administrators.

This chapter reviews books and scholarly articles published within the last 15 years, except for literature related to the foundation of cultural competence in various disciplines. The literature used for this review was found primarily through the FIU Library and online databases (e.g. EBSOHost, Emerald Insight, ERICProQuest, and JSTOR). Additional literature was sought through inquiry of public administration professors with research expertise in the areas of diversity and cultural competence. Key search terms used for locating literature included: cultural competence, cultural competence in public administration, cultural competence framework, cultural competence education, cultural competence predictors, cultural competence theory, and cultural competence research. 
Though several disciplines have generated a significant amount of research related to the definition, measurement, and predictors of cultural competence, the discipline of public administration has superficially covered this topic in terms of practical application, without development of a testable theoretical foundation. This study aimed to address this gap in the literature through answering the following main research questions:

1. How is cultural competence measured in public administration?

2. What are the predictors of cultural competence for public administrators?

This literature review begins with an overview and history of cultural competence. Presented next is a review of the development of cultural competence within the field of public administration, as well as the gaps in the literature that exist. This chapter concludes with a synthesis of how this dissertation advances the investigation of cultural competence within public administration. The purpose of this chapter is to outline the current state of cultural competence research within public administration and to demonstrate how this study fills significant gaps in the public administration literature related to both theory and practice.

\section{Cultural Competence Defined}

Cultural competence is "a set of congruent behaviors, attitudes, and policies that come together in a system, agency, and among professionals and enable that system, agency, or those professionals to work effectively in cross-cultural situations" (Cross et al., 1989). When further broken down, culture refers to both internal and external characteristics such as "thoughts, communications, actions, customs, beliefs, values" and 
race, ethnicity, gender, religion, and age, among others (Cross, 1989, p.7). Competence refers to the ability to function successfully in a given context.

\section{Brief History of Cultural Competence}

The concept of cultural competence is rooted in healthcare's patient-centeredness model (Saha, Beach, \& Cooper, 2008). From this model, cultural competence arose as a means to overcome the barriers that cultural and linguistic factors caused in a health care setting; and its expansion driven by data which supported the premise that there was a gap in the quality of care received by minority vs. majority patients. In addition to the doctor-patient relationship, cultural competence in healthcare was also concerned with systemic causes of healthcare disparities.

This focus on cultural competence expanded into several additional disciplines, which led to significant contributions related to how cultural competence is both conceptualized and operationalized. The scholars behind these contributions include Cross et al. (1989), who established the most well-known and cited framework; and Camphina-Bacote (2002), Sue (2001), and Van Dyne \& Ang (2006) are among other scholars that have developed conceptual frameworks for cultural competence in their respective disciplines of nursing, psychology, and business.

In addition to advancing the conceptualization of cultural competence, professional organizations and accrediting bodies now include cultural competence in their professional codes of ethics or discipline-specific accreditation requirements, demonstrating that it is a desirable set of skills for students to develop and professionals to maintain. These organizations include, but are not limited to, the Council for Accreditation of Counseling \& Related Educational Programs, the Council on Social 
Work Education, the American Library Association, the Association to Advance Collegiate Schools of Business, the American Psychological Association, the Association of Schools and Programs of Public Health, the Association of American Medical Colleges, and the American Association of Colleges of Nursing (Association of American Medical Colleges, 2005; American Association of Colleges of Nursing, 2009; American Psychological Association, 2013; Council on Education for Public Health, 2013; Rubaii \& Calarusse, 2012, p. 235). Due to the early adoption of the concept by these fields, scholars have had the opportunity to develop a strong theoretical and empirical foundation for cultural competence. This has validated its position within medicine, nursing, and other related fields.

\section{Development of Cultural Competence in Public Administration}

Although several disciplines have extensive experience with cultural competence research and practice, public administration faced several hurdles in its acceptance as a valid area of study (Rice, 2007b). This section of the literature review will examine the challenges faced in the development of cultural competence within public administration, as well as important responses to these challenges. An overview of the specific integration of cultural competencies within public administration education and a proposed method of its integration within public organizations follows. The chapter concludes with a review of the pertinent gaps in the literature and how this current study aims to fill those gaps. 


\section{Challenges to Cultural Competence Development in Public Administration: The Traditional School of Public Administration}

Dependence on the bureaucratic ethos paradigm of professional ethics, a remnant of traditional public administration, is an overarching theme in the delay of incorporating cultural competence into public service delivery (Rice, 2007a). Per Goss (1996), the professional ethics of public administrators aligns with either a bureaucratic ethos or a democratic ethos. Several critical public administration models prefaced the bureaucratic ethos, including Weber, Wilson, Taylor, and Goodnow and Willoughby, "who found the ethos consistent with the study of comparative administration and the application of rationalism" (Goss, p. 578). Denhardt (1997), describes the bureaucratic ethos, and its related ideals of "efficiency, economy, standardization, hierarchical authority structures, accountability systems, impartiality, and subservience to political superiors", to promote good government (p. 1093). As opposed to the democratic ethos, these bureaucratic ideals are tied to the "legitimacy" of public administration, and thus, these values define what it means to be a professional public servant (Denhardt, 1997). This bureaucratic ethos, however, is in direct opposition to what cultural competence requires of public servants. Two values in particular, neutrality and discretion, have hindered the acceptance of cultural competence as a valued set of skills.

The values of neutrality and discretion are closely linked to the politicsadministration dichotomy. This dichotomy, coined by Wilson (1887), posits that politics and administration be kept separate, and that politics determined the "who" and "what" of policymaking, while the administration oversaw the "how" of implementing policies. Per 
Overeem (2005), while the politics-administration dichotomy does not apply to current public administrators, the value of neutrality has remained critically important.

According to Kaufman (1956), the concept of neutral competence is part of the "traditional school" of public administration, and was established out of a need to reign in aspects of the government that allowed for political interests to trump the interests of the citizens. Neutrality is a value entrenched within public administration, as demonstrated by the requirement that a wide range of regulating bodies remain in charge of creating objective policy. Furthermore, the traditional school of public administration necessitated limited discretion (Denhardt \& Denhardt, 2000). As with neutrality, public administrators value administrative discretion in public administration, since it reduced "governmental machinery" (e.g. legislative session length), to "limit the amount of harm they could do" (Kaufman, 1956, p. 1060). The value placed on neutral competence and limited discretion led to the slow development of social equity, and more specifically cultural competence, as important areas of focus within public administration (Rice, 2007; Carrizales, 2010; Frederickson, 2005).

The dependence of bureaucratic, rather than democratic ethos impacts several other areas of study within public administration. Examples of these areas are social and emotional intelligence, and a focus on virtue-based ethics as opposed to teleological or deontological ethics. Social intelligence, defined as interpersonal effectiveness; and emotional intelligence, defined as "the capacity to exert emotional labor" (Mastracci, Newman, \& Guy, 2010) are two elements within public administration that require a focus on democratic ethos. Virtue ethics calls for public servants to develop certain character traits such as courage and benevolence, and gauge an ethical decision based on 
how it evidences these traits, as opposed to simply following rules or making sure to meet the "bottom-line". According to Newman, Guy, and Mastracci (2009), "the most important challenge facing public administrators is not to make work more efficient but to make it more humane and caring" (p. 6). Ensuring that public administrators are culturally competent also contributes to furthering the development of social and emotional intelligence, as well as encouraging decision-making based on what is most beneficial for the public and indicative of good virtues.

\section{Responding to Cultural Competence Development Challenges in Public Administration: New Public Administration and New Public Service}

The traditional school of public administration and its related values contributed to the avoidance of topics such as cultural competence while it was under development in other fields. However, a movement to New Public Administration and New Public Service, established a focus on alternative values (Frederickson, 2005; Denhardt \& Denhardt, 2000); which in turn allowed for topics that conflicted with traditional values to be taken seriously. The chapter first outlines cultural competence's relationship to social equity and New Public Administration. Next, the chapter argues the relationship between cultural competence and its link to New Public Service and its focus on democratic, as opposed to bureaucratic values.

New Public Administration and Social Equity. The development of cultural competence in public administration is tied closely to the social equity movement that was accelerated during the 1968 Minnowbrook conference. Although the Minnowbrook conference served as an impetus to take social equity into sincere consideration, it is not the first mention of social equity in the discipline. Frederickson (2005) traces social 
equity back to one of public administration's formative figures, Henri Fayol, and his fourteen general principles; as well as Woodrow Wilson and the "The Study of Administration". However, the Minnowbrook conference allowed for the expansion of the concept, leading to the creation of the New Public Administration (NPA) paradigm.

The main purpose of the conference was to reflect on the state of public administration and discuss the future of the field (O’Leary, Van Slyke, \& Kim, 2011). While Minnowbrook did not solely focus on social equity, it served as the starting point for NPA, "based on a call for bureaucrats to become an instrument for achieving social equity" (Gooden \& Portillo, 2011, p. 162). One of the most important outcomes of discussing social equity at this conference was the recognition that public administrators are not "value neutral" and that they must constantly seek to reconcile the five values of "responsiveness, worker and citizen participation in decision making, social equity, citizen choice, and administrative responsibility" (Frederickson, 2010, p. 40). In an essay penned after the conference, Marini summarizes the impact of NPA by stating "but surely the pursuit of social equity in public administration is no more a holy grail than the objectives of educators, medical doctors, and so forth. Still, it appears that new public administration is an alignment with good, or possibly God" (as cited in Frederickson, 1980, p. 20). Thus, the first Minnowbrook Conference played a critical role in recognizing social equity as a pillar of public administration.

NPA signaled a shift away from a sole focus on the three original pillars of public administration: efficiency, effectiveness, and economy. It called for an enhanced advocacy of all citizens, ensuring that public administrators were working for the 
common good of the people (Frederickson, 2010). The definition for social equity used in this dissertation is as follows:

The fair, just and equitable management of all institutions serving the public directly or by contract; the fair, just and equitable distribution of public services and implementation of public policy; and the commitment to promote fairness, justice, and equity in the formation of public policy (National Academy of Public Administration, 2010).

The movement towards NPA and the championing of social equity was a critical factor in demonstrating that alternative values, which supported the development of cultural competence as a component of social equity, are equivalent to the traditional values of economy, efficiency, and effectiveness.

New Public Service: Changing Values. According to Denhardt (1997), "both bureaucratic and democratic ideals are essential elements of the public administration ethos, yet these two sets of ideals have not been effectively integrated into an ethic of public administration" (p. 1091). In contrast to the bureaucratic values present in the early stages of public administration, both New Public Administration and New Public Service focus on democratic values such as: fairness, justice, equity, upholding the public interest, and responsiveness (Denhardt, 1997; Goss, 1996). New Public Service focuses on serving the public interest, being accountable to multiple stakeholders (including community values), and encourages discretion if there are accountability measures in place (Denhardt \& Denhardt, 2000); which encourages a focus on social equity and cultural competence. This shift in values, especially in terms of a decreased focus on 
neutrality and discretion, elevated social equity as a critical element of public service delivery.

\section{Fostering Cultural Competence Development within Public Administration}

This change from increased value placed on the bureaucratic ethos to one which embraces more democratic ideals, paved the way for social equity and cultural competence to develop within public administration. This portion of the literature review focuses on how this development occurred, beginning with the shift from diversity towards cultural competence. First, the chapter presents a review of how public administration education programs integrate cultural competence, followed by an exploration about how organizations adapt to it. A review of how to measure cultural competence within public administration is then presented. Finally, a synthesis of the chapter reflects on the current state of cultural competence and how this dissertation seeks to fill the gaps that exist within the literature.

From Diversity to Cultural Competence. Both the New Public Administration and New Public Service movements triggered a focus on improved public service delivery outcomes in favor of the public interest. This dissertation argues that cultural competence is social equity in action. However, before scholars accepted cultural competence, diversity and diversity management were previous models to operationalize social equity. As such, it is important to outline this transition. Per Bailey's (2010) representative bureaucracy continuum, the movement towards cultural competence occurred in four phases: Equal Employment Opportunity (EEO), affirmative action, managing diversity, and cultural competence. 
EEO and affirmative action fall under the umbrella of diversity, with a strict focus on creating policies that were non-discriminatory and encouraged recruitment of historically discriminated-against groups; however, organizations adopted an assimilation model which did not embrace cultural differences, but instead expected employees to adapt (Bailey, 2010). In terms of managing diversity, scholars prefer a synergistic model instead of assimilation. This model encompasses "systematic and planned programs or procedures that are designed to improve interaction among diverse people, especially people of different ethnicities, genders, and cultures" (Mathews, 2010, p. 214). Though managing diversity was a significant step forward, it focused primarily on "how to structure the organization in terms of human resources... [whereas] the transition to cultural competence addresses the behavior aspects of interacting with diverse coworkers and citizens" (Cram \& Alkadry, 2018).

The final stage, cultural competence, has an expected outcome that is "fairness and results oriented [,] seeks to end discrimination in service provision, [and] held accountable for meeting policy guidance" (Bailey, 2010, p. 179). Following the focus of New Public Service, cultural competence considers the citizen's needs as the driving factor for policy and practice. Furthermore, the organization must be equipped to address “client/employee culture mismatches and employee needs" (p.179) as well provide training to employees to ensure that they have the skills to work with each other as well as with citizens. According to Lopez-Littleton and Blessett (2015), "cultural competency is regarded as an essential component of promoting social equity in public service delivery and is the manner in which issues of diversity are responded to and addressed in the public sector" (p. 558), which demonstrates the statement that cultural competence is 
social equity in action. Based on this transition from diversity to cultural competence, it is important to understand how public administration scholars have suggested its integration at the educational and organizational level.

Cultural Competence Integration and MPA Programs. One of the proposed methods of increasing cultural competence education and training is to enhance its integration across the MPA curriculum. The accrediting body of schools of public administration, the Network of Schools of Public Policy, Administration, and Affairs (NASPAA), requires programs to ensure their graduates can "communicate and interact productively with a diverse and changing workforce and citizenry" ("Standard 5", 2009). Although this does not directly reference the term "cultural competence", it demonstrates a commitment to the spirit of the term. Although NASPAA recognizes the importance of being able to interact with a diverse workforce and citizenry, studies have found that many institutions do not regard it as a main component of the program of study (White, 2004; Rubaii \& Calarusse, 2014). The NASPAA accreditation process recently began to require institutions to submit assessment plans for all the required universal competencies; however, institutions are only required to present a full cycle of evidence for three competencies of their choice. Research in this area has indicated that the competencies chosen are usually more traditional in nature; as the assessment of cultural competence can be challenging. The next section of the literature review explores the state of cultural competence integration within MPA programs, and explores the challenges related to achieving integration.

Cultural competence consists of awareness, knowledge, and skills; each of these constructs requires students to display certain competencies from lower to higher levels 
of thinking. The Cultural Competence Continuum developed by Cross et al. (1989) demonstrates the various levels that individuals experience on their journey to becoming culturally competent. Therefore, cultural competence is a developmental process, and it is important to have competencies that relate to each of these development stages, so that they are introduced, reinforced, and assessed (Norman-Major, 2012). The notion of cultural competence as a developmental process will help MPA program understand that coverage of cultural competencies throughout the curriculum will help to create connections between how social equity and other more traditional focuses of public administration, such as performance measurement, are related "to one another in both a qualitative and quantitative sense, in academic and practical environments" (LopezLittleton \& Blessett, 2015, p. 560).

One of the leading studies on the inclusion of cultural competence across the curriculum comes from Susan Perry White's dissertation (2005). The focus of her study was to identify the extent to which MPA programs "provide cultural competency training to students through their curricula" (p. ii). Her findings indicated that, after a review of top public administration program's syllabi of diversity-related courses, less than half of the programs' core courses included diversity-related topics. Her recommendations include the need for programs to recognize the importance of cultural competence in the curriculum; identify key knowledge, attitudes, and skills related to cultural competence; and to develop a core course related to diversity and cultural competence. She mentions the importance of faculty support and assessment of learning outcomes to ensure the sustainability of the new curriculum. To expand upon White's work, this dissertation seeks to identify specific discipline-wide cultural competencies that can help faculty 
achieve these broader goals of curriculum integration and creation of a stand-alone course.

There are several case studies in the literature regarding cultural competence integration within the MPA curriculum however, the authors of these studies note the challenge of curriculum integration (see Norman-Major \& Gooden, 2012). Several frameworks provide guidance. For example, Lewis, Lewis, \& Williams’ (2012) developed a framework for programs to follow, in which they identify the state of their program and then leverage strengths and improve on weaknesses that they discover related to cultural competence. Several researchers presented in-depth curriculum frameworks that describe cultural competence topics and the specific core classes to address them in (Carrizales, 2010; Norman-Major, 2012). Norman-Major (2012) also provides a guideline for the assessment process, starting with the creation of learning outcomes through designing the assessments. However, to follow these frameworks, program faculty must be knowledgeable about cultural competence and understand best practices for its assessment.

One of the many challenges related to integrating cultural competence throughout the curriculum is a lack of a disciplinary-wide definition (Rubaii \& Calarusse, 2012). Although there is sufficient literature to guide a program through the cultural competence curriculum development and assessment cycle, difficulty may arise, as there has not been research to determine what these cultural competencies should be. Curriculum redesign to include cultural competencies requires significant effort on the part of the faculty and the department must ensure proper faculty training (Borrego \& Johnson, 2012, p. 283). In addition to the time intensive nature of curriculum redesign, acquiring buy-in from 
tenured faculty may be difficult; finding adequate time to provide comprehensive cultural competence training, and procuring resources to conduct this training, may be additional challenges (Lopez-Littleton \& Blessett, 2015). Therefore, this type of curriculum integration requires a large investment on the part of the program.

This dissertation addressed the challenge to elevate the dialogue of which cultural competencies programs should integrate into their curriculum and how to measure these competencies. The goal was to not only understand the level of cultural competence among future public administrators, but also to help programs understand how to integrate the identified competencies across their curriculum. As this study identified competencies, integration can be more easily facilitated across the curriculum, allowing for a more effective learning experience (Norman-Major, 2012).

\section{Development of Cultural Competence within the Public Organization.}

Analogous to the development of cultural competence within MPA programs, its integration into organizations faces similar challenges. In terms of formal recognition within the field, public administrators should demonstrate cultural competence. As stated in the American Society for Public Administration's (ASPA) code of ethics, public administrators have a professional obligation to "Strengthen social equity. Treat all persons with fairness, justice, and equality and respect individual differences, rights, and freedoms. Promote affirmative action and other initiatives to reduce unfairness, injustice, and inequality in society." And although the Practices to Promote the ASPA Code of Ethics (2013) does not directly define cultural competence, it states that public administrators should "oppose all forms of discrimination and harassment and promote 
affirmative action, cultural competence, and other efforts to reduce disparities in outcomes and increase the inclusion of underrepresented groups".

Per Rice and Mathews (2012), organizations that successfully operate within the framework of cultural competence have "improved the quality and delivery of programs and services to constituents and clients" (p. 20). The major contributors to cultural competence research regarding public organization; Rice, Mathews, and Bailey, have made significant recommendations as to how to best facilitate, incorporate, and evaluate this set of skills. Next, a review of how cultural competence enhances service delivery precedes an overview of how organizations can assess their current level of cultural competence and implement change.

According to Rice (2007b), public administrators who demonstrate cultural competence can be more effective in serving the public's diverse needs. Responsiveness to citizens is a value championed by New Public Service perspectives of public administration. Elias and Alkadry (2011) argue that citizens and public administrators have the most interaction at the "front lines" where service delivery occurs. If both parties feel empowered during this interaction, negotiation between needs and capabilities takes place. They argue that the focus on a public administrator's level of effectiveness and empowerment is important, as it dictates how to meet the citizens' needs. In terms of increasing effectiveness and empowerment, a culturally competent approach to public service delivery would encourage a public administrator to contextualize each encounter, therefore improving effectiveness. In addition to providing important contextual information, culturally competent organizations are better able to 
identify and address disparities within a community, ensure that their services are relevant to their citizen base, and prepare public servants to do their jobs (Rice, 2007a).

Legal mandates and evidence-based research encouraged the transition from diversity to cultural competence, on the premise that it led to better organizational outcomes (Bailey, 2005). Challenges for public organizations to becoming culturally competent are linked to the dearth of research on the topic within the field, and the lack of understanding that cultural competencies are essential to public service delivery (Rice, 2010; Mathews, 2010); whereas “other fields, such as the behavioral sciences, see diversity and cultural competencies as necessary for the effective delivery of programs and services" (Mathews, 2010, p. 221).

To further research and practice in this area, Rice (2007b) developed a framework for public organizations, in addition to a self-assessment tool. His framework establishes eight critical areas to cover cultural competence for the organization to have success: organizational culture, governance, administration, policy and decision making, service delivery, marketing and community relations, personnel practices, and communications. To assess success in each of these areas, he has also derived a self-assessment checklist that organizational leaders can use. Mathews (2010) sought to identify whether these types of self-assessments were practical for organizations and if they led to increases in cultural competence. Her research demonstrated that organizations can successfully use self-assessment to improve their level of cultural competence; however, her findings indicated that scholars and practitioners do not view cultural competence as a developmental process, and that several competencies that are critical to service delivery were not viewed as important. Most importantly, Mathews (2010) states, "our failure to 
teach cultural competence's theories, tools, and techniques is a disservice to the citizens we serve" (p. 248); thus, it becomes critical to ensure that public administrators receive training, either through their education or at the organizational level.

To establish cultural competence as the norm within the public organization, it is critical to ensure that programs that are preparing the nation's future public administrators provide sufficient coverage (Rice \& Mathews, 2010). This dissertation seeks to fill these gaps in the literature by providing a set of competencies specific to public administration and identifying predictors of cultural competence to further enhance education and training programs.

Measurement of Cultural Competence within Public Administration. Authors have made several suggestions for assessment of cultural competence within public administration at the MPA and organizational level (Rice, 2007; Bonilla et al., 2012; Norman-Major, 2012). However, it is important to explore how public administration has approached measurement outside of individualized or programmatic assessments. A formal instrument for measuring the cultural competence of public administrators did not exist prior to the start of this dissertation. However, in 2015, Longoria and Rangarajan developed an indirect assessment instrument based on a review of the literature. Their scale consisted of a combination of existing scales from outside fields (18/24 items) and author-created items (6/24 items). Based on their analyses, their findings indicate while three of the four indexes indicate reliable results (with an alpha coefficient of above .7), more research must focus on "the eventual development of a valid and agreed upon cultural competence instrument" (p. 14). Although the authors published this scale 
concurrently with this study, this dissertation uses a more rigorous methodology to develop an indirect assessment scale in conjunction with a direct assessment measure.

Synthesis. Based on a review of the literature, cultural competence development has undergone significant strides within public administration. Due to the difficulty of integrating these competencies into the MPA curriculum, and lack of intentional empirical focus, discussion surrounding cultural competence primarily takes place it public administration education-based forums. This dissertation aims to close this gap through understanding how to define cultural competence within public administration, and what competencies should be an important focus at both the educational and organizational training level. Furthermore, advances empirical research on the topic through identifying predictors of cultural competence for future public administrators.

\section{Summary}

The field of public administration is behind other social science disciplines in recognizing the importance of cultural competence as a set of skills that are critical for public service (Rice, 2007b). Most of the scholarly work on the topic focuses on how to integrate these skills within the curriculum; however, there is not an agreed upon definition of cultural competence for public administration, or a set of competencies that can facilitate this integration (Berry-James, 2012). In terms of measurement, only one study exists (Longoria \& Rangajaran, 2015); however, this study used a majority of competencies derived from other disciplinary assessments, which may not present an accurate representation of what cultural competencies are most important for public administrators. Based on the review of the literature there were three significant gaps that this dissertation aimed to fill. First, it defined cultural competence for public 
administration. Second, it identified the competencies that culturally competent public administrators should be able to demonstrate. Finally, predictors of cultural competence for future public administrators were identified. The findings from this dissertation will inform curricular integration, organizational training programs, and future empirical research on cultural competence within public administration. 


\section{CHAPTER 3}

\section{CONCEPTUAL FRAMEWORK AND THEORETICAL FOUNDATION}

This chapter elaborates upon the research questions, conceptual framework, and theoretical foundation that guide this study, as well as the related hypotheses tested. The chapter first outlines the research questions and then presents the conceptual framework, followed by a presentation of the related hypotheses. The chapter ends with an exploration of the theoretical foundation and related hypotheses.

\section{Research Questions}

The main goal of this dissertation was two-fold. The first goal was to identify a definition of cultural competence for public administrators and determine what competencies public administrators should be able to demonstrate. The second purpose was to identify predictors of cultural competence for graduating MPA students. The research questions address several important gaps in the literature, most notably a lack of a discipline specific definition of cultural competence; the non-existence of an agreed upon set of cultural competencies for public administrators; and the exploration of appropriate measures of cultural competence for MPA students. The specific research questions that guided this study were:

1. How is cultural competence measured in public administration?

2. What are the predictors of cultural competence for public administrators?

\section{Conceptual Framework and Hypotheses}

The conceptual framework presented below guided the first research question, and related sub-questions, of the study. Cross' Model of Cultural Competence is first reviewed; as it guided the dissertation in terms of viewing cultural competence as a 
developmental process, informed the design of the PACCI rubric, and was used to interpret the results of the study in Chapter 7. This chapter then presents the public administration specific cultural competence framework developed for this study.

\section{Cross' Model of Cultural Competence}

This study was guided by Cross et al.’s 1989 framework for cultural competence to improve service delivery. This framework is used widely in the study of cultural competence for several disciplines, and serves as the guiding framework for the National Center for Cultural Competence, a leader in the development of best practices regarding cultural and linguistic competence. Cross' model includes the cultural competence continuum; the culturally competent system of care; developing cultural competence; service adaptation; and planning for cultural competence.

\section{Cultural Competence Continuum. This Cultural Competence Continuum} consists of six stages, including “1) cultural destructiveness, 2) cultural incapacity, 3) cultural blindness, 4) cultural pre-competence, 5) cultural competency and 6) cultural proficiency" (Cross, 1989). The purpose of the continuum is to demonstrate that cultural competence is a developmental process. Each level of the continuum is based on three elements: attitudes, policies, and practices. At one extreme of the continuum, cultural destructiveness is when these attitudes, polices, and practices are destructive to a culture (e.g. cultural genocide). At the other extreme, cultural proficiency indicates that a system holds cultures "in high esteem" (e.g. through institutionalization of cultural knowledge; research and dissemination of results related to effective cultural approaches). For an institution or agency to reach this level, it is necessary that all relevant stakeholders, at all levels, participate in moving these attitudes, policies, and practices in a positive direction. 
Culturally Competent System of Care. The second element of Cross' model is the Culturally Competent System of Care. This type of system "values diversity, has the capacity for self-assessment, is conscious of the dynamics inherent when cultures interact, has institutionalized cultural knowledge, and has developed adaptations to diversity" (p. 34). The goal of these five steps is to ensure that agencies are "accessible, acceptable, and available" (p. 39), with the overarching goal is to demonstrate that by making a system more responsive to non-majority populations, it becomes enhanced for everyone.

Developing Cultural Competence. The third element of Cross' model is Developing Cultural Competence. He argues that development occurs at the policymaking level, administrator level, practitioner level, and consumer level. To reach the sixth level of the cultural competence continuum, it is critical for each of these agency levels to work together in developing the attitudes, policies, and practices that foster a culturally competent system. Wilson's (1982) competencies, listed by Cross et al. (1989), are significant to this dissertation; these competencies are divided into three areas: personal attributes, knowledge, and skills. Cross et al. note that these competencies are developed through training.

Service Adaptations. The fourth element of Cross' model is service adaptation. Cross states that "the delivery of effective services cross-culturally requires that existing services be adapted to fit the needs of the targeted minority group or individual. The possible ways of adapting services are endless" (p. 55). Although Cross' model provides examples for service adaptations specific to the mental health service delivery, public administration practitioners can use these as models for other public service-specific 
adaptations. To successfully adapt programs and services, the agency must ensure that they are not approaching the adaptation process from a "color-blind perspective", as successful agencies will consider individual cultural contexts to best serve their surrounding communities.

Planning for Cultural Competence. The last step of Cross' model involves actual implementation of cultural competence initiatives to achieve the goals set forth previously. Cross notes that strategic planning is necessary for effective implementation and that the following five steps are critical for success: assessing the environment, developing support, resource development, leadership development, and mission and action. Approaching implementation with these steps helps agencies realize that change can occur and that the change process is manageable.

Synthesis. When Cross developed his model in the late 1980's, he noted that "the field lacks standards in this area, because it has yet to define what one should know to be competent in serving minority clients. In addition, the system lacks incentives for the development of cross cultural skills" (p. 22). This dissertation approached cultural competence in public administration from this same perspective, seeking to develop a public administration specific definition, and establishing the competencies public administrators should be able to demonstrate. Furthermore, it used Cross' model as a guide, in Chapter 7, for how these competencies can be better integrated into education and training programs to foster the development of culturally competent public service agencies. The following section of this chapter illustrates a public administration specific conceptual framework, which elaborates upon research conducted on cultural competence within public administration, as well as other relevant models. 


\section{A Public Administration Conceptual Framework for Cultural Competence}

In addition to the guidance that Cross' model provides, this study combined frameworks from Rice and Mathews (2012), Carrizales (2010), and the Tilford Group (2001). This section first presents a brief overview of the framework, followed by a detailed review of each framework's components. Figure 1 presents the conceptual model.

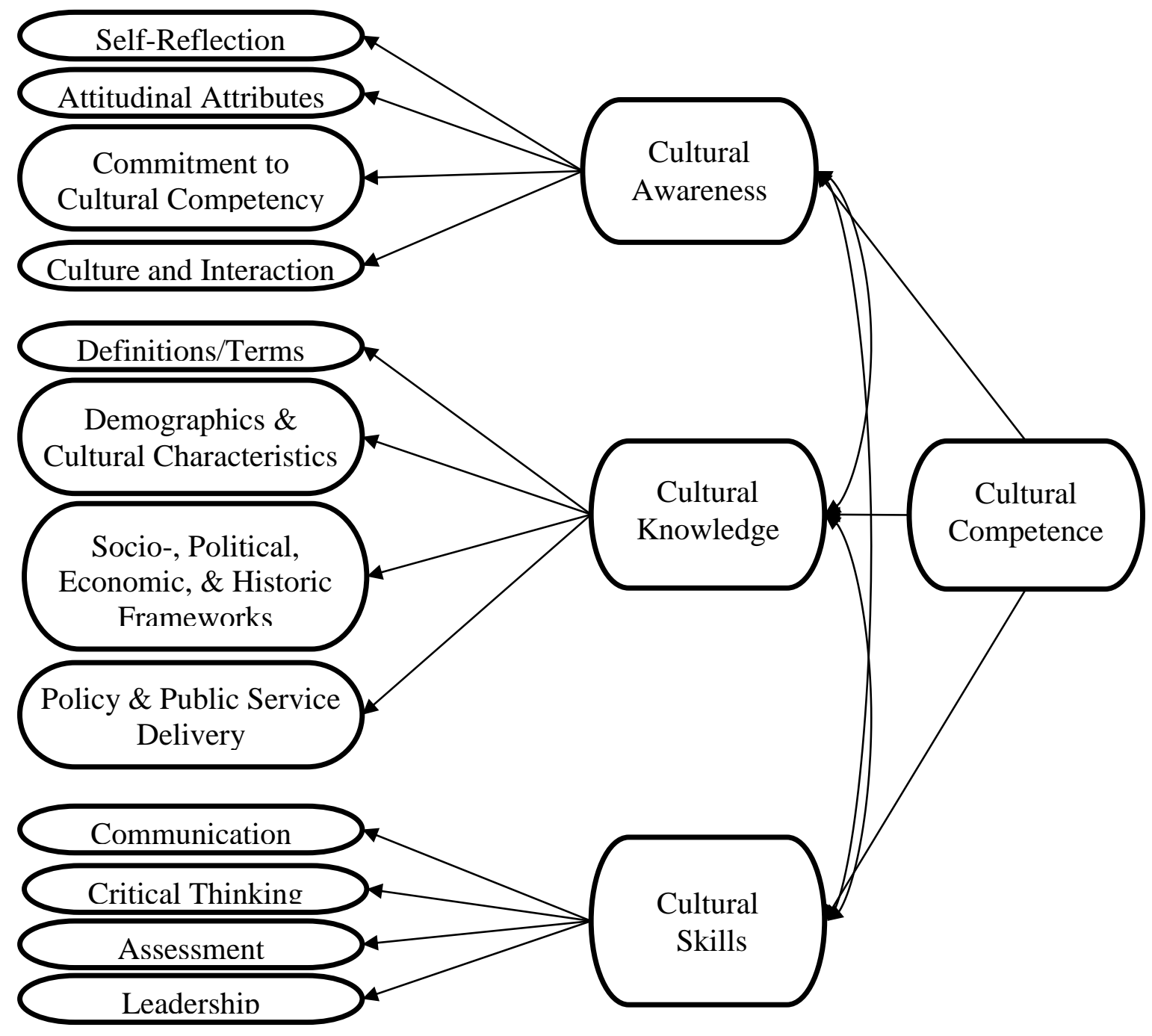

Figure 1 Conceptual Model for Cultural Competence. Adapted from Carrizales, 2010; Cross et al., 1989; Rice \& Mathews, 2012; Tilford Group, 2001 
For this study, cultural awareness was defined as "those values, attitudes, and assumptions essential to working with clients and service recipients who are culturally different from a public agency service delivery professional" (Rice and Mathews, 2012, p. 27). This study defined "culturally different" to include race, gender, socioeconomic status, sexual orientation, language, and additional cultural characteristics including "generational difference, religion, politics, and technology" (Borrego \& Johnson, 2012).

Following awareness, cultural knowledge was defined as "understanding the worldviews of various cultural groups and possessing knowledgeable professional expertise relevant to persons in other cultures. Public agency service delivery professionals must gather information about cultural groups they are working with and learn in what ways cultural constructs influence how these groups respond to the helping process" (Rice \& Mathews, 2012, p. 27).

Finally, cultural skills were defined as "those attributes that allow public service agency delivery professionals to effectively apply cultural awareness and cultural knowledge they have learned" (Rice \& Mathews, 2012, p. 27). It is also important to note the relationship between these three components, as demonstrated in the model. Rice and Mathews (2012) expand upon this relationship, stating that by "not having a foundation of cultural awareness and cultural knowledge make it difficult to possess cultural skills" (p. 27). The following section examines each of the awareness, knowledge, and skills components more closely.

Cultural Awareness. Cultural awareness comprises four components: selfawareness/reflection, an understanding of how culture shapes interactions, attitudinal attributes, and a personal commitment to cultural competence values (Rice \& Mathews, 
2012; Carrizales, 2010; Tilford Group, 2001). Figure 2 illustrates the components of cultural awareness.

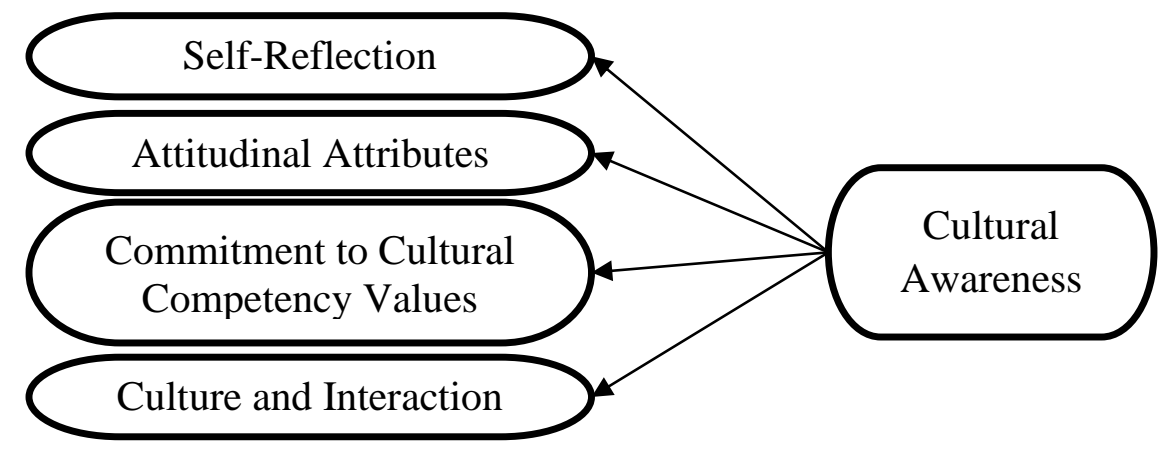

Figure 2 Cultural Awareness Components. Adapted from Rice \& Mathews, 2012; Tilford Group, 2001

Table 1 provides the conceptual definition for each of these latent variables and its relationship to cultural awareness.

Table 1

Cultural Awareness Components: Conceptual Definitions

\begin{tabular}{|l|l|}
\hline \multicolumn{1}{|c|}{ Component } & \multicolumn{1}{c|}{ Conceptual Definition } \\
\hline Self-Reflection & $\begin{array}{l}\text { Self-reflection encompasses an individual's ability to be aware } \\
\text { of one's cultural identity and how this identity affects } \\
\text { development, and perspectives, values, and perceptions (Rice \& } \\
\text { Mathews, 2012; Tilford Group, 2001). }\end{array}$ \\
\hline $\begin{array}{l}\text { Attitudinal } \\
\text { Attributes }\end{array}$ & $\begin{array}{l}\text { These are "traits needed by those who live and work in a diverse } \\
\text { world" (Tilford Group, 2001). These traits include flexibility, } \\
\text { respect, empathy, openness, and acceptance of other cultural } \\
\text { perspectives, and the willingness to change perspectives as } \\
\text { necessary (Rice \& Mathews, 2012; Tilford Group, 2001). }\end{array}$ \\
\hline $\begin{array}{l}\text { Commitment to } \\
\text { Competence Values }\end{array}$ & $\begin{array}{l}\text { A commitment to cultural competence values means that an } \\
\text { individual demonstrates “a strong commitment to justice, social } \\
\text { changes, and social equity" and is aware of how these concepts } \\
\text { influence a person's view of service delivery (Rice \& Mathews, } \\
\text { 2012). }\end{array}$ \\
\hline $\begin{array}{l}\text { Culture and } \\
\text { Interaction }\end{array}$ & $\begin{array}{l}\text { Culture and Interaction refers to an individual's ability to } \\
\text { understand how cultural characteristics influence the } \\
\text { communication process and can impact what occurs during } \\
\text { delivery of services (Rice and Mathews, 2012). }\end{array}$ \\
\hline
\end{tabular}


Cultural Knowledge. Cultural knowledge builds upon the first construct of awareness. Per Rice and Mathews (2012), the acquisition of awareness signals that one is willing "to consider various worldviews, perspectives, and cultural differences" (p.27); this "willingness" moves towards "understanding" these views and perspectives as well as gaining knowledge to better serve those of a different culture. Cultural knowledge is comprised of the four following components: definitions/terms, demographics \& cultural characteristics, "socio-, political, economic, and historic frameworks", and policy and public service delivery (Rice \& Mathews, 2012; Carrizales, 2010; Tilford Group, 2001). Figure 3 illustrates these components.

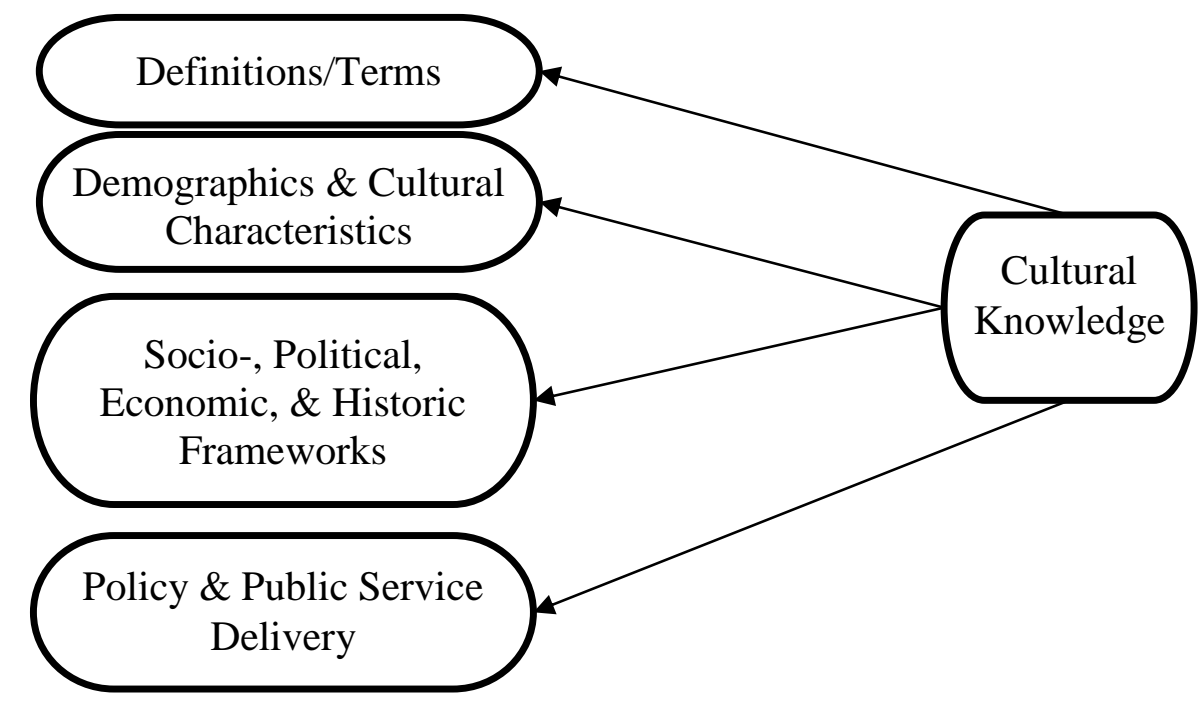

Figure 3 Cultural Knowledge Components. Adapted from Carrizales, 2010; Rice \& Mathews, 2012; Tilford Group, 2001

Table 2 provides the conceptual definition for each of these components. 
Table 2

Cultural Knowledge Components: Conceptual Definitions

\begin{tabular}{|l|l|}
\hline \multicolumn{1}{|c|}{ Component } & \multicolumn{1}{c|}{ Conceptual Definition } \\
\hline Definitions/Terms & $\begin{array}{l}\text { Definitions/Terms refer to an individual's ability to define cultural } \\
\text { competence and have a normative understanding of definitions/terms } \\
\text { related to cultural competence within public administration } \\
\text { (Carrizales, 2010). }\end{array}$ \\
\hline $\begin{array}{l}\text { Demographics \& } \\
\text { Cultural }\end{array}$ & $\begin{array}{l}\text { Knowledge of demographics and cultural characteristics relates to an } \\
\text { individual's ability to have knowledge about different cultures and } \\
\text { their related characteristics, such as language, perspectives on service, } \\
\text { and perceptions of other cultures. In addition, it requires knowledge of } \\
\text { changing demographics and how this impacts future service delivery } \\
\text { (Rice \& Mathews, 2012; Carrizales, 2010; Tilford Group, 2001). }\end{array}$ \\
\hline $\begin{array}{l}\text { Socio-, Political, } \\
\text { Economic, \& }\end{array}$ & $\begin{array}{l}\text { Knowledge of Socio-, political, economic, and historic frameworks } \\
\text { relates to an individual's ability to understand how these frameworks } \\
\text { impact culture and relations between cultures (Rice \& Mathews, 2012; } \\
\text { Frameworks }\end{array}$ \\
\hline $\begin{array}{l}\text { Policy \& Public } \\
\text { Service }\end{array}$ & $\begin{array}{l}\text { Knowledge of policy and public service delivery relates to an } \\
\text { individual's ability to understand federal and state policies related to } \\
\text { cultural competence and how this impacts public service delivery. It } \\
\text { also refers to an ability to have knowledge about the policy-making } \\
\text { process and how cultural competence plays a role in policy } \\
\text { development (Carrizales, 2010). }\end{array}$ \\
\hline
\end{tabular}

Cultural Skills. To adequately demonstrate cultural skills, cultural awareness and knowledge must first be present. Building on the willingness to consider other cultures and the ability to learn about and understand these cultures, cultural skills require that one can "decide on culturally sensitive and culturally appropriate interventions and strategies" (Rice \& Mathews, 2012, p.17).

The four components of cultural skills focus on goal of ensuring that public service professionals can deliver necessary services to their stakeholders in a culturally appropriate way. Figure 4 illustrates the cultural skills components. 


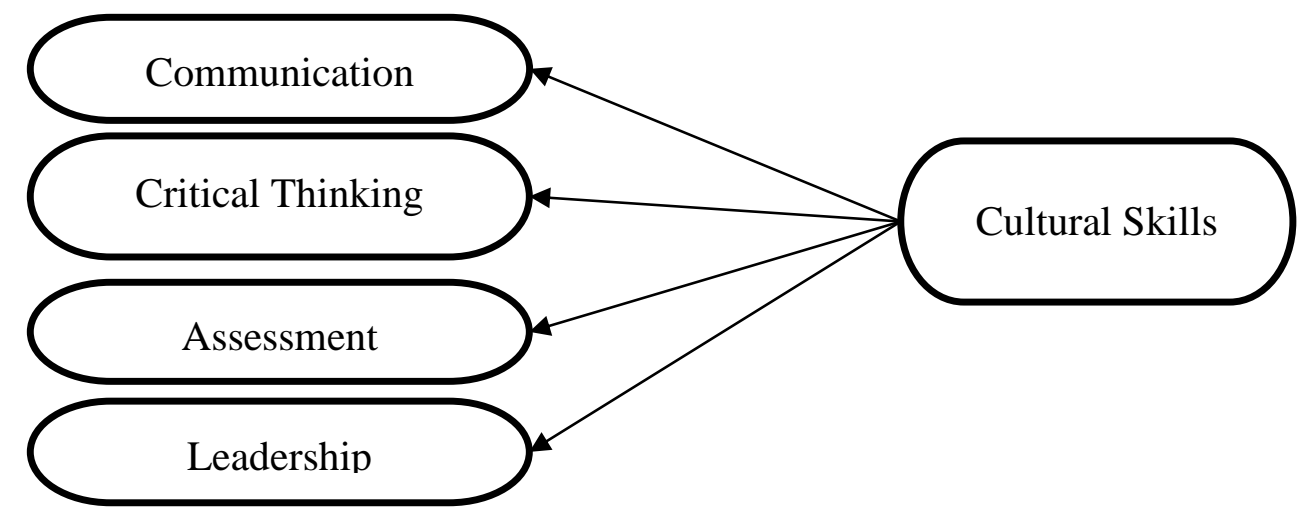

Figure 4 Cultural Skills Components. Adapted from Carrizales, 2010; Rice \& Mathews, 2012; Tilford Group, 2001

Table 3 provides the conceptual definition for each of these components.

\section{Table 3}

Cultural Skills Components: Conceptual Definitions

\begin{tabular}{|l|l|}
\hline \multicolumn{1}{|c|}{ Component } & \multicolumn{1}{c|}{ Conceptual Definition } \\
\hline Communication & $\begin{array}{l}\text { Cultural communication relates to an individual's ability to } \\
\text { demonstrate appropriate "verbal and nonverbal skills in } \\
\text { interaction with those that are culturally different" from them } \\
\text { (Tilford Group, 2001). }\end{array}$ \\
\hline Critical Thinking & $\begin{array}{l}\text { Critical thinking relates to an individual's ability to apply } \\
\text { cultural awareness and knowledge skills to improve service } \\
\text { delivery (e.g. culturally sensitive interventions, reasoning to } \\
\text { understand diverse perspectives) (Rice \& Mathews, 2012; } \\
\text { Tilford Group, 2001). }\end{array}$ \\
\hline Assessment & $\begin{array}{l}\text { Assessment requires an individual to be able to accurately } \\
\text { assess the context and outcomes of a public service delivery } \\
\text { encounter and can assess one's own level of cultural } \\
\text { competence "skills, comfort level, growth, and development" } \\
\text { (Rice \& Mathews, 2012). }\end{array}$ \\
\hline Leadership & $\begin{array}{l}\text { Leadership requires the ability to lead culturally diverse groups, } \\
\text { lead culturally sensitive interventions, and provide leadership in } \\
\text { creating programs that take culture into account (Rice \& } \\
\text { Mathews, 2012; Tilford Group, 2001). }\end{array}$ \\
\hline
\end{tabular}




\section{Hypothesis}

The hypothesis for the first research question relates to the measurement of cultural competence for public administrators. The conceptual framework presented in this dissertation posits that cultural competence in public administration consists of three sub-dimensions: cultural awareness, cultural knowledge, and cultural skills. This conceptualization is cross-disciplinary in nature and was first developed for use in the field of psychology (Cross et al., 1989; Sue, 2001). Public administration scholars such as Rice and Mathews (2007a) and Carrizales (2010) have developed models that include these three dimensions. To truly demonstrate cultural competence, these three dimensions must be present simultaneously. Rice and Mathews (2012), highlight the important interdependence of these areas, as "not having a foundation of cultural awareness and cultural knowledge makes it difficult to possess cultural skills that allow for once to decide on culturally sensitive and culturally appropriate interventions and strategies" (p. 27). To answer the first research question, this dissertation sought to develop both indirect and direct assessment measures that can test the stated conceptual framework. The first hypothesis related to this research question focuses on whether cultural competence in public administration is comprised of three sub-dimensions.

H1: Cultural Competence is comprised of three sub-dimensions: cultural awareness, cultural knowledge, and cultural skills.

The second hypothesis is related to whether or not the direct and indirect measures for this study are positively correlated.

H2: The direct and indirect measures of cultural competence will be positively correlated. 
Figure 5 presents the hypothesized model.

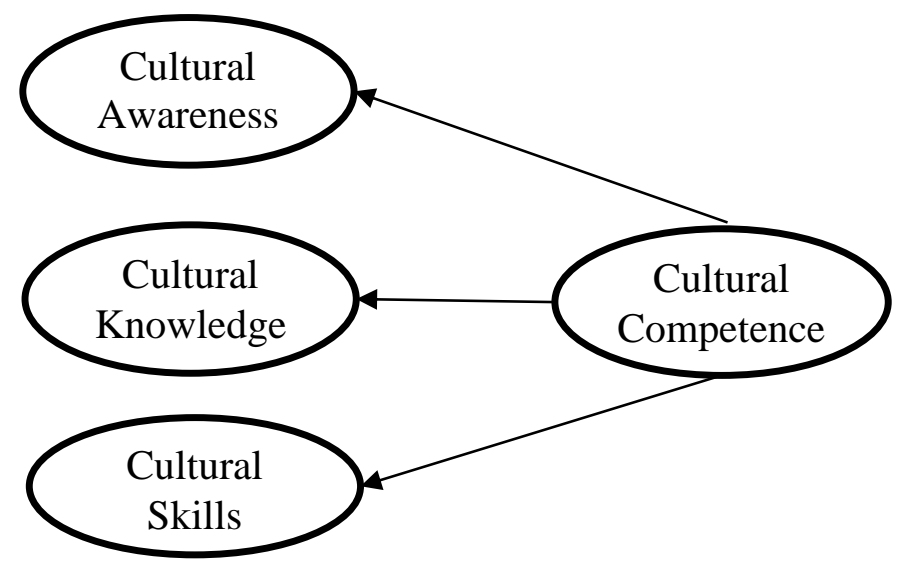

Figure 5 Hypothesized PACCS Model

\section{Theoretical foundation and Hypotheses}

The theoretical foundation presented below guides the second research question: What are the predictors of cultural competence for future public administrators? The predictors chosen for this study are: PSM, MPA exposure to diversity, color-blind racial attitudes, and Lifetime Experience with Discrimination. Figure 6 demonstrates the predicted relationship between these predictor variables and cultural competence for graduating MPA students. 


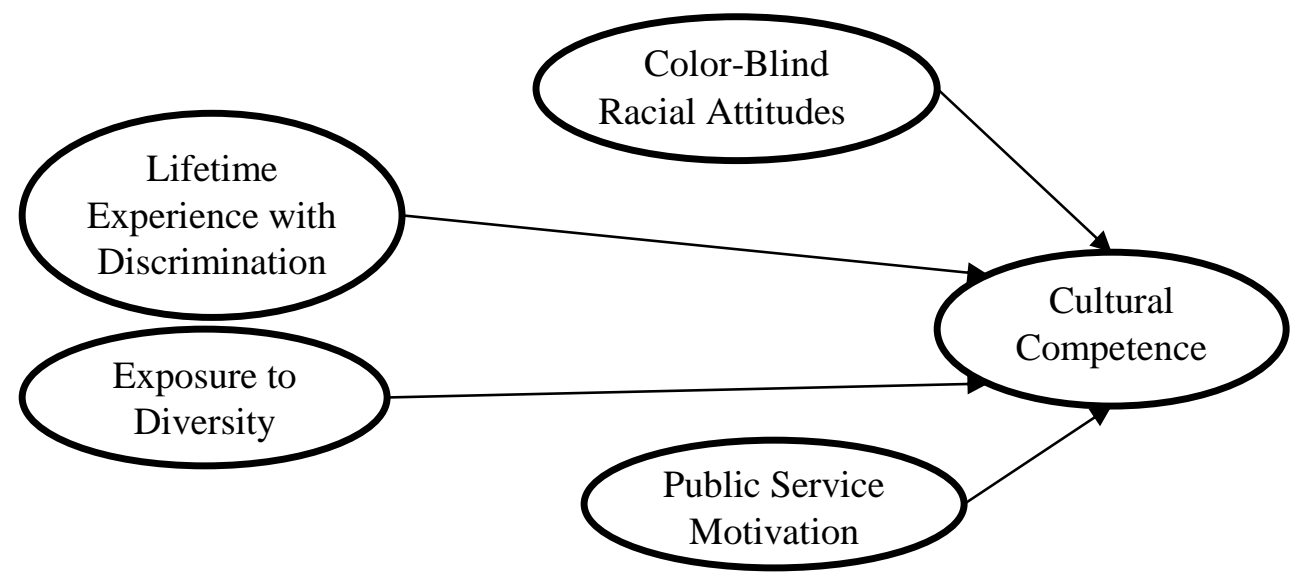

Figure 6 Predicted Relationship between Predictor Variables and Cultural Competence of Graduating MPA Students

Each of the identified predictors and related hypotheses are described below.

PSM. PSM refers to "an individual's predisposition to respond to motives grounded primarily or uniquely in public institutions" (Perry, 1996, p.5). PSM theory developed because of a belief that people who entered public service had different rational, norm-based, and affective motives than those who work in the private sector. The motives that Perry identified are: attraction to policy making, commitment to the public interest, social justice, civic duty, compassion, and self-sacrifice (p.10-11). Further, studies have demonstrated that higher levels of PSM are associated with ethical behavior and increased charitable acts (Choi, 2004; Houston, 2006). These motives are closely tied to the characteristics one must possess to demonstrate cultural competence (Camphina-Bacote, 2008; Papadopolous et al., 2015, Rice \& Mathews, 2012; Vera \& Speight, 2003; Sue, 2001).

The motives of commitment to the public interest, desire to serve the public interest (civic duty) and social justice are most closely associated with the cultural awareness component of "commitment to cultural competency values" (Perry, 1997; Rice 
$\&$ Mathews, 2012; Vera \& Speight, 2003). The motives of compassion and self-sacrifice align with the cultural awareness component of "attitudinal attributes" (Perry, 1997; Papadopolous et al., 2015). Each of these cultural awareness components relate to an internal commitment to social equity and social justice, both central components to PSM and cultural competence.

While PSM describes the motivations of why people enter the public sector as opposed to the private sector, the concept of cultural desire describes the driving force behind the development of cultural competence. According to Camphina-Bacote (2002), cultural desire begins the process of cultural competence, as it refers to motivation in the form of a provider demonstrating genuine interest in providing culturally responsive services. In other words, their motivation is driven by wanting to help the client/patient and not an obligation to help. This dissertation posits that the motivational bases that underlie PSM are congruent with the concept of cultural desire, as PSM indicates that a public administrator's "commitment to a program may emanate from a genuine conviction about its social importance" (Perry \& Wise, 1990, p. 369).

Due to the relationship between PSM and cultural desire, this dissertation posits that PSM is positively related to cultural competence. If a person has a high level of PSM, they would also have a high level of cultural competence, as the motivators embedded within PSM are in line with cultural desire (Camphina-Bacote, 2008).

H3: A person with higher levels of PSM will have higher levels of cultural competence.

MPA Exposure to diversity: Multicultural/Diversity training and classroom diversity. According to the literature, "cultural incompetence is presumed to arise from a 
lack of exposure to and knowledge of the Other, and also from individual biases, prejudices, and acts of discrimination" (Kumas-Tan et al., 2007). Furthermore, many diverse organizations and academic programs tout their level of diversity; whether this diversity impacts outcomes, however, is important to understand. Therefore, exploring the relationship between exposure to diversity and cultural competence will help to better define how organizations can leverage diversity to improve outcomes. This dissertation explores exposure to diversity through multicultural/diversity training and classroom diversity.

Several studies found that multicultural training has a positive impact on cultural competence outcomes (Benkert et al, 2011; Price., 2005; Kardong-Edgren et al., 2005; Sodowsky et al., 1998; Crandall et al., 2003; Schim, Doorenbos, \& Borse, 2005; Schim, Doorenbos, \& Borse, 2006). Specifically, the studies found that training impacted the outcomes of both non-minority and minority participants (Benkert et al., 2011). However, future research should focus on the most effective types of training, as well as include more rigorous and sound methodologies for exploring this relationship (Ekblad, Martilla, \& Emilsson, 2000; Price et al., 2005). Scholars from the health fields conducted most of the research regarding training; as such, it is important to examine if these conclusions are true for the field of public administration.

Higher education research notes that classroom diversity, defined as "the incorporation of information about diverse groups in the curriculum" (Pike \& Kuh, 2006, p. 427) has a positive relationship with learning outcomes. Students who are exposed to these diversity-related activities, experience positive gains in areas related to "increased openness to diversity and challenge... [and] greater racial/culture knowledge and 
understanding and commitment to social justice" (Terenzini, et al., 2001). Additional studies demonstrate the positive impact that enrollment in diversity courses can have on development of one's social agency, a characteristic that is important to cultural competence (Laird, 2005; Gurin et al., 2002). Studies related specifically to cultural competence also demonstrated positive relationships between curricular integration and improvements in cultural competence scores (Caffrey et al., 2005; Sargent, Sedlak \& Martsolf, 2005).

Based on the relationship between cultural competence and exposure to diversity through training or classroom curriculum, this dissertation posits that taken together, exposure to diversity has a positive relationship with cultural competence outcomes.

H4: People with higher levels of multicultural training and classroom diversity will have higher levels of cultural competence.

Color-Blind Racial Attitudes. Color-blind Racial Ideology is "a dominant racially based framework that individuals, groups, and systems consciously or unconsciously use to justify the racial status quo or to explain away racial inequalities in the United States" (Neville, Spanierman, \& Doan, 2006, p. 276). This ideology stems from the theory of modern racism, which focuses on the ambivalence of White American's "attitudes and behavior toward Black Americans" (McConahay, 1981, p. 551). However, due to this theory's focus on Black Americans, it was critical to develop an ideology and corresponding scale which reflected the multicultural society that now makes up the United States (Neville et al., 2000). The importance of the color-blind racial ideology is that it acknowledges the important role that culture plays in the lives of individuals (Tarca, 2005). 
In terms of color-blind racial attitudes and cultural competence, it is critical for public administrators to take cultural factors into account, inclusive of race, when constructing policy or engaging in service delivery (Rice, 2007b). As noted in Cross et al.'s (1989) continuum, cultural blindness refers to the third stage of development, demonstrating that it is not compatible with the characteristics demonstrated by a culturally competent organization or individual. Furthermore, research demonstrates that higher levels of color-blind racial attitudes are negatively correlated with cultural competence outcomes (Neville, Spanierman, \& Doan, 2006; Spanierman, Poteat, Wang, \& Oh, 2008; Chao, Good, Flores, \& Wei, 2010).

Based on the relationship between cultural competence and color-blind racial attitudes, this dissertation posits that color-blind racial attitudes are negatively correlated with cultural competence.

H5: People with higher levels of color-blind racial attitudes will have lower levels of cultural competence.

Lifetime Experience with Discrimination. The inclusion of this question draws upon literature related to the relationship between experiences with discrimination and cultural competence. One area of research posits that exposure to racism and the stress related to racism led to an increase in social involvement (Mattis et al., 2004). While much of this research has focused on African Americans, the concept of using negative experiences with discrimination to foster positive outcomes is important to explore.

Previous research on the topic indicates that "such experiences ultimately result in negative self-perceptions, and force individuals to engage in pro-social behaviors as a means to repair the self- concept" (as cited in White, 2008, p. 119); however, other 
interpretations have been found, such that these experiences lead to "a sense of agency to maintain and improve conditions for a community of people who are often disregarded by institutions structures and mainstream society" (White, 2008, p. 120). Kumas-Tan et al. (2007), highlight that cultural competence requires a person to be cognizant of whether their actions, or inactions, "support the status quo and business as usual unintentionally, but systematically, privilege some and marginalize others" (p. 554). This dissertation argues that personal experience with this marginalization, would translate into a better understanding of how to avoid marginalizing others - thereby improving one's cultural competence.

H6: People with more Lifetime Experience with Discrimination will have higher levels of cultural competence.

The above hypotheses serve to set the stage to explore a possible theoretical foundation for the cultural competence of graduating MPA students.

\section{Summary}

The conceptual framework outlined in this chapter expands upon previously developed frameworks about how to conceptualize cultural competence within the context of public administration. The proposed conceptual framework informed the Delphi study and subsequent analysis of the direct and indirect measures to provide a full answer to research question one. The theoretical foundation served to build upon existing literature from other disciplines about possible predictors of cultural competence, while expanding upon this literature to include public administration specific items. The following chapter describes the methodology used to answer the proposed research questions. 


\section{CHAPTER 4}

\section{METHODS}

The purpose of this study was to define cultural competence within public administration, and identify the predictors of cultural competence for future public administrators. The methods used for this study addressed the following two research questions and related sub-questions:

1. How is cultural competence measured in public administration?

a. How is cultural competence defined in public administration?

b. What cultural competencies should public administrators be able to demonstrate?

c. What are direct and indirect measures of cultural competence that can be used to measure a public administrator's level of cultural competence?

2. What are the predictors of cultural competence for public administrators? This chapter first outlines the research design and rationale the study. Next, it presents a description of the methodology, including participant selection, data collection, and operationalization of the dependent and independent variables. It ends with a review of the data analysis procedures.

\section{Research Design and Rationale}

The main dependent variables for this study are the direct and indirect measures of cultural competence. The main independent variables are PSM, CoBRAS, Exposure to Diversity, and Lifetime Experience with Discrimination. To answer the research questions, the study employed a sequential two-phase mixed methods design. Mixed 
methods research provides the opportunity to engage in the process of "induction, deduction, and abduction"; thus, lending to the robustness of the research design (Johnson \& Onwuegbuzie, 2004). Through integrating both qualitative and quantitative methodologies, this study makes several contributions to the existing literature on cultural competence within public administration. The first phase of the study employs both qualitative and quantitative methodologies to answer research question 1. Specifically, it employs a modified Delphi method to answer research question 1a and 1b; and confirmatory factor analysis to answer question 1c. The second phase of the study uses quantitative methodology to answer research question 2, through Ordinary Least Squares (OLS) regression analysis. Figure 7 provides a broad overview the methodology used for this study.

\begin{tabular}{|c|c|c|}
\hline Measurement of $C$ & $\begin{array}{c}\text { PHASE } 1 \\
\text { ural Competence in Pub }\end{array}$ & dministration \\
\hline $\begin{array}{l}\text { Purpose } \\
\text { A. Determine how } \\
\text { cultural competence } \\
\text { is defined within } \\
\text { public } \\
\text { administration and } \\
\text { identify cultural } \\
\text { competencies } \\
\text { required of public } \\
\text { administrators. } \\
\text { B. Validate indirect } \\
\text { and direct } \\
\text { measurement of } \\
\text { cultural competence } \\
\text { for public } \\
\text { administrators }\end{array}$ & $\begin{array}{l}\text { Variables } \\
\text { A. Cultural } \\
\text { competencies } \\
\text { developed from a } \\
\text { review of the } \\
\text { literature } \\
\text { B. Indirect and Direct } \\
\text { measures of } \\
\text { cultural competence } \\
\text { for public } \\
\text { administrators. }\end{array}$ & $\begin{array}{l}\text { Data Analysis } \\
\text { A. Content Analysis, } \\
\text { A priori coding, } \\
\text { Modified Delphi } \\
\text { method, } \\
\text { Descriptive } \\
\text { Statistics } \\
\text { B. Confirmatory } \\
\text { Factor Analysis }\end{array}$ \\
\hline
\end{tabular}




\begin{tabular}{|c|c|c|}
\hline \multicolumn{3}{|c|}{$\begin{array}{l}\text { PHASE } 2 \\
\text { Predictors of cultural competence for Public Administration }\end{array}$} \\
\hline \begin{tabular}{l}
\multicolumn{1}{c}{ Purpose } \\
Analyze whether PSM, \\
MPA Exposure to \\
Diversity, Social \\
Desirability, Lifetime \\
Experience with \\
Discrimination, and Color- \\
Blind Racial Attitudes are \\
predictors of cultural \\
competence for future \\
public administrators.
\end{tabular} & $\begin{array}{l}\quad \text { Variables } \\
\text { DV: Direct and Indirect } \\
\text { Measures of Cultural } \\
\text { Competence } \\
\text { IV: PSM, Exposure to } \\
\text { Diversity, and Color-Blind } \\
\text { Racial Attitudes }\end{array}$ & \begin{tabular}{l}
\multicolumn{1}{c}{ Data Analysis } \\
Descriptive Statistics and \\
OLS Regression
\end{tabular} \\
\hline
\end{tabular}

Figure 7 Methodology Used for this Study

To summarize the methodology, the first phase of the study consists of a modified Delphi method, which is "a method for structuring a group communication process so that the process is effective in allowing a group of individuals, as a whole, to deal with a complex problem" (Linstone \& Turloff, 2002, p.3). Scholars in the disciplines of nursing, business management, and education have used Delphi studies to identify cultural competence models (Jirwe et al., 2009; Senyshyn, 2002; Sprott, 2014). Therefore, this serves as an appropriate methodology to answer the first research question of this study.

The Delphi method uses a facilitator to structure communication across a panel of experts to achieve consensus "through a series of structured questionnaires" (Hasson et al., 2000; Skulmoski, Hartman, and Krahn, 2007). According to Rowe \& Wright (1999), there are four characteristics of a Delphi study: "anonymity, iteration, controlled feedback, and the statistical aggregation of group response" (p. 354). Traditional Delphi studies begin with an unstructured first round, allowing the experts in the field to voice any issues related to the topic that they feel are important (Rowe \& Wright, 1999); 
however, this study is a modified Delphi due to the inclusion of a structured first round. A structured round was determined to be most efficient, due to the availability of competencies from other disciplines, as well as literature on the selection of these competencies. Per Hsu \& Sandford (2007), "it should be noted that it is both an acceptable and a common modification of the Delphi process format to use a structured questionnaire in Round 1 that is based upon an extensive review of the literature" (p. 2); this is the only modification to the Delphi process for this study. The Delphi method usually consists of three rounds, or until the panel reaches consensus (Skulmoski, Hartman, and Krahn, 2007). The modified Delphi study for this dissertation required two rounds to achieve consensus, and a third round to confirm the results. The results of the Delphi informed and developed the two dependent variables of the study.

The second phase of the study uses OLS regression to answer the second research question. The goal of OLS is to reduce the sum of the squared errors, while estimating a linear model. OLS is appropriate for this study, as the dependent variables are measured on an interval scale; and the model can account for multiple explanatory variables (Hutcheson, 2011).

\section{Methodology}

This section of the dissertation outlines the participants, data collection procedures, operationalization of variables, instrumentation, describes the pilot studies conducted, and outlines the data analysis plan.

\section{Participants}

This study required two separate sampling strategies. Phase 1A included a purposive sample of experts in cultural competence and diversity training. Phase 1B 
included a random sample of NASPAA accredited institutions within the USA. The following section of the dissertation describes the sampling strategy and participants for each phase. It concludes with a description of the human subjects considerations for this dissertation.

Delphi Sampling and Participants. The modified Delphi phase of the study required purposive sampling. The criteria for inclusion were experience with diversity or cultural competence; participation in diversity committees; or participation in the NASPAA's Commission on Peer Review and Accreditation. In addition to identifying participants through a review of public administration literature regarding diversity and cultural competence; a review of members who participated on the commission and the NASPAA Diversity and Social Equity committee provided leads.

The final sample included nineteen experts. The experts received an invitation letter, which included a description of the study and the relevant inclusion criteria. In total, 18 experts in the areas of diversity or cultural competence agreed to participate in the study. Twenty-one percent of the sample was male, and 79\% female; in terms of rank, $44 \%$ held the rank of associate professor, $28 \%$ held the rank of full professor, and $28 \%$ held the rank of assistant professor; $39 \%$ of the participants were directors for their respective programs.

Student survey sampling and participants. Graduating MPA students are the units of the analysis for the study. The sampling frame for phase 1B is NASPAA accredited institutions. The population for the study is graduating students of NASPAA accredited MPA programs; for 2012-13, this number was 4,981 (NASPAA, 2014). To estimate a sample size range, the conducted a-priori analysis used a desired confidence 
level of $95 \%$ in conjunction with a desired power of .8. Using these numbers, an appropriate sample size would be a minimum of 131 students (Soper, 2017). To draw the sample, a list of NASPAA accredited institutions with their number of degrees awarded in 2013-14 was downloaded from the NASPAA website and divided into 9 census regions to ensure diversity of institutions. The exclusion criteria for the study consisted of any institution outside of the United States, any institution that did not award any degrees in 2013-14, and any institution that does not award an MPA degree. Inclusion criteria consisted of whether the institution offered a capstone class to graduating students. The sampling frame included a total of 136 institutions.

After the researcher divided the institutions by region, she used Excel to generate a proportional random sample from each region. To achieve an adequate sample size, two rounds of invitation letters were sent out. Thirty-two institutions received the first round of invitations; 17 received the second round. Out of the 49 institutions invited to participate, 19 institutions agreed, and out of these 19, 14 returned the surveys. The represents a response rate of $28.6 \%$. In total, the 14 participating institutions returned 267 individual student surveys. Table 4 indicates each region's ideal proportion of the sampling frame in relation to the proportion of regions included in this study's sample, the p-value associated with this difference, and the number of student participants from each region. The $\mathrm{p}$-values indicate that the ideal proportion and sample proportion of institutions selected for the study are not significantly different at the .05 level. These pvalues demonstrate support for the study's external validity. 
Table 4

Ideal Regional Sample Proportion and Actual Regional Sample Proportion

\begin{tabular}{llll|l}
\hline Region & $\begin{array}{l}\text { Ideal Proportion } \\
\text { Institutions }\end{array}$ & $\begin{array}{l}\text { Sample } \\
\text { Proportion } \\
\text { Institutions }\end{array}$ & $\begin{array}{l}\text { P-value for } \\
\text { Difference }\end{array}$ & $\begin{array}{l}\text { Student } \\
\text { Participants }\end{array}$ \\
\hline West South Central & $10.30 \%$ & $7.14 \%$ & .668 & 13 \\
East South Central & $10.30 \%$ & $7.14 \%$ & .668 & 9 \\
Mountain & $6.06 \%$ & $7.14 \%$ & .886 & 19 \\
Pacific & $13.94 \%$ & $7.14 \%$ & .346 & 3 \\
New England & $3.03 \%$ & $7.14 \%$ & .592 & 30 \\
South Atlantic & $23.64 \%$ & $35.71 \%$ & .076 & 82 \\
Middle Atlantic & $12.12 \%$ & $14.29 \%$ & .766 & 69 \\
East North Central & $13.94 \%$ & $7.14 \%$ & .346 & 23 \\
West North Central & $6.67 \%$ & $7.14 \%$ & .950 & 19
\end{tabular}

\section{Data Collection}

Delphi method data collection. Nineteen participants received round 1 of the survey. The response rate for round 1 was $94.44 \%$. One participant did not return the survey and one of the participants only partially completed the Round 1 survey.

Seventeen participants received Round 2 of the survey. The response rate for round two was $88.24 \%$. One participant declined to participate due to time constraints and one participant did not provide reasons for why they did not wish to participate.

The modified Delphi included three rounds of data collection. Qualtrics was used to design and distribute the questionnaires for each round. A pilot test occurred before each round of the Delphi to address issues of clarity and ease of use. IRB approval was sought and obtained prior to piloting or administering the survey. Each participant consented to participation in the study. 
Two experts, one in assessment and one in diversity/cultural competence, agreed to participate in the pilot study for the Delphi survey. These experts agreed to provide feedback regarding the following questions:

1. Are the instructions clear?

2. Was the survey presented in a reasonable format?

3. Were any of the questions presented as "leading" or "biased"?

4. How long did it take you to complete the survey?

5. Please indicate any additional comments or suggestions.

The primary concern of the reviewers was length of the original instrument. The original instrument included the 60 distilled competencies; however, the reviewers indicated that this format was lengthy. After reviewing the feedback, only the 17 higher order competencies were included for round 1 . Reviewers suggested minor edits to the instructions and formatting. The experts also reviewed round 2 of the survey; however, they did not suggest any edits.

Round one of the Delphi study consisted of the panel members reviewing the competencies and definition of cultural competence. For round one, panel members ranked whether a competence was relevant to the field on a nine-point scale. In addition, panel experts provided feedback regarding both the competencies and the definition. Participants received 2 reminder emails to complete the survey.

The questionnaire for round two was drafted based on the quantitative results and qualitative responses from the first Delphi round. Participants received the new list of competencies and ranked each competency on a nine-point scale. Participants received reminder emails to complete the survey and had a timeline of 2 weeks to respond. Panel members reviewed the new definition that incorporated feedback from round 1 . They indicated their level of agreement and provided additional feedback if necessary. 
Round three confirmed the results. Participants reviewed the results of the modified Delphi and provided any additional feedback within two weeks. There was no feedback received and the round three results were finalized.

Although research demonstrates that it is difficult to achieve full reliability within Delphi studies, several techniques, such as continuous verification of data, help to demonstrate reliability (Skulmoski, Hartman, and Krahn, 2007). In terms of validity, the use of experts within the field help to enhance content validity and the use of more than one round increases concurrent validity (Hasson, Keeney, \& McKenna, 2000). In this study, the researcher verified the data and ensured adequate participation of experts after each round to maximize the validity and reliability of the Delphi method.

Student survey data collection. The student survey data were collected via hardcopy and online surveys, if there was not an in-person capstone class. The researcher mailed/e-mailed consenting program directors the survey and/or Qualtrics survey link, and a copy of the IRB approved informational instructions. Program directors returned the surveys via a prepaid envelope. Program directors also filled out an informational survey on their program to collect information about how they assess cultural competence within their program. The program director survey information informed Chapter 6 of the dissertation. Each participating program director received a confirmation email after the surveys were mailed. Program directors received reminders at set intervals throughout the semester.

Eighteen Executive MPA students piloted the student survey. Students took the survey, and reflected on the clarity of the instructions, order of the questions, and their overall impression of the survey. The original survey consisted of three short answer 
questions, followed by the 3 scales to measure the independent variables, and ended with demographic questions. The students felt that overall, the survey was too long, and that the 3 short answer questions were repetitive. In addition, they said that the instructions should more clearly state that all answers would be anonymous. They also recommended that the scale questions come before the short answer questions.

In addressing the feedback from the pilot study, the researcher re-worded the instructions and changed the word "anonymous" to bold font. Changing the order of the survey was not possible, as some of the scale items included reference to cultural competence. Exposure to this term, in addition to items that indicate the definition of the term could bias the answers to the short-answer questions.

To address the length of the survey and the perceived repetitive nature of the short-answer questions, the rubric, designed prior to the pilot study, was used to assess each answer. The assessment procedure demonstrated that two of the questions were very similar in nature, so one of the questions was then removed from the survey. In addition to the above changes, the pilot also provided the opportunity to test the rubric in terms of ease of use and phrasing of the categories. The researcher made minor grammatical and editorial changes to the rubric to provide greater clarity between each of the categories. The final rubric is presented in Appendix A.

\section{Instrumentation and Operationalization of Variables}

This study used both previously validated and newly constructed instruments. Phase 1A and 1B included the use of survey instruments. Phase 1B also employed two newly constructed scales for the dependent variables in the study. In addition, the independent variables consist of two previously validated scales, as well as a third scale 
developed for this study. The following sections describe each of the instruments used in the study.

Delphi survey. Phase 1A consisted of a modified Delphi method to identify the competencies that a culturally competent public administrator should be able to demonstrate. To construct the survey items for the first round of the Delphi, a literature review was conducted to determine the main constructs of cultural competence and related competencies. The literature review revealed three main constructs (cultural awareness, cultural knowledge, and cultural skills), and additional latent variables related to each of these constructs. The conceptual framework described in Chapter 2 outlines these constructs.

The content analysis performed on six existing frameworks led to the identification of 221 competencies. These competencies then mapped on to each of the 12 latent variables. A total of 60 competencies were distilled from the 221 during this process. After the pilot study demonstrated that 60 competencies led to survey fatigue, the final round one Delphi included 17 higher-order competencies.

Student survey. The student survey was designed to collect data on the relevant dependent and independent variables needed for analysis in Phase 2. The Delphi survey informed the development of dependent variables in the study. The independent variables consisted of two previously validated scales, and one scale developed for this study. The following section summarizes the survey instrument, and describes the operationalization of the dependent, independent, and control variables.

The unit of analysis for this study is the graduating MPA student. Thus, a survey is the optimal tool for collecting data. The survey consisted of two case studies, requiring 
short answer responses, followed by multiple choice questions related to the independent variables, and ended with demographic questions.

Operationalization of dependent variables. The dependent variables for the study are the Public Administration Cultural Competence Index (PACCI) and the Public Administration Cultural Competence Scale (PACCS). The PACCI is a direct measure, while the PACCS is an indirect measure of student learning. A direct measure "requires students to display their knowledge and skills as they respond to the instrument itself"' (Palomba \& Banta, 1999, p. 11); whereas indirect measure measures "such as surveys and interviews ask students to reflect on their learning rather than to demonstrate it" (Palomba \& Banta, 1999, p.12). In terms of cultural competence assessment, indirect measures have received most of researchers' attention. Despite the availability of cultural competence self-assessments (indirect measure), a direct measure of cultural competence is necessary for public administration educators to understand the extent to which their students are achieving.

PACCI. The PACCI is an index derived from the rubric scores of the student's short answer responses. The score is derived from a rubric comprised of three main constructs (awareness, knowledge, and skills) and four rating categories (Proficient, Competent, Beginner, and Novice). A rubric was a critical component for the assessment of these responses, as they serve to create an objective assessment of student learning (Bresciani et. al, 2009; Hansson et al., 2014). Each rubric category aligned with the results of the modified Delphi method in phase 1A and a review of relevant cultural competence rubrics developed at other institutions. The two case studies are presented below. 
Case study 1. You are a director of client services and have noticed that there has been an increase in a new population of immigrants seeking services. Your coworkers are uncomfortable with this new population and are frustrated that appointments with these clients take twice as long to complete due to communication barriers. The clients are also frustrated with the service they receive.

1. What is the conflict in this situation? (1-2 Sentences)

2. What individual and organizational characteristics attributed to this conflict? (1-2 Sentences)

3. How can this conflict be resolved at both the employee and organizational level? (1-2 Sentences)

Case Study 2. You are an admissions officer at a local community college. Your next client is a young woman who would like to enroll in some courses. Her husband also attends the meeting with her. You begin to ask questions about her interests and why she would like to enroll in courses, but her husband answers for her. You think to yourself that this man is too controlling and that he should not speak for his wife. You say to the husband: "I am speaking to your wife, please don't answer for her." The couple gets up and leaves.

1. What is the conflict in this situation? (1-2 Sentences)

2. What factors attributed to this conflict? (1-2 Sentences)

3. As the admissions officer, how do you assess the situation and how do you ensure that this conflict does not happen again? (1-2 Sentences)

Each case study measured a student's level of cultural knowledge, awareness, and skills. However, for this study, the first case measured lower order skills, while the second case study measured higher order skills. While the first question primarily deals with how surface characteristics can influence service delivery; the second case approaches cultural competence from a perspective that explores various aspects of culture, including gender role assumptions, in addition to communication. In the construction of these two case studies, the hypothesis was that answers to the first case study would come from a delivery perspective, without the student reflecting on how their own assumptions would affect their answer; however, with the second question, the 
hypothesis was that the students would have a harder time reigning in their own biases and assumptions about culture, power within relationships, or gender roles.

The PACCI rubric was validated through the Delphi survey and review of similar cultural competence related rubrics. Furthermore, experts in the field reviewed the case studies to ensure clarity of the instructions and questions. In terms of reliability for rubric instruments, interrater reliability (IRR) is crucial, as it "provides a statistical estimate of the extent to which two or more judges are applying their ratings in a manner that is predictable and replicable" (Stemler, 2004, p. 9). There are several forms of interrater reliability, with the most popular being percent agreement; however, this is an unacceptable form of IRR since it "do[es] not correct for agreements that would be expected by chance and therefore overestimate the level of agreement" (Hallgren, 2012, p.4). Instead of percent agreement, Cohen's Kappa and Intraclass Correlation Coefficient are two of the most accepted means to compute IRR with the ICC most appropriate for ordinal data. To determine interrater reliability of rubric scores, the Intraclass Correlation Coefficient (ICC) were calculated on a random sample of 60 student case studies, which is approximately $13 \%$ of the case studies assessed in the study. Two raters evaluated each case study in the sample. The first rater is the author of this dissertation, the second rater is an assessment professional with experience in diversity. The minimum number of raters required for conducting interrater reliability analysis is two.

The Intraclass Correlation Coefficient is appropriate for this sample, as it is "suitable for studies with two or more coders, and may be used when all subjects in a study are rated by multiple coders, or when only a subset of subjects is rated by multiple coders and the rest are rated by one coder" (Hallgren, 2012, p. 9). ICC ranges from 0 to 1 , 
with 1 indicating perfect agreement. The ICC consists of two measures: single and average.

A two-way mixed effects model was used to estimate the ICC for each rubric category and the total score for both case studies. Prior to conducting the IRR study of the sample, the researcher and selected rater completed a norming session. The raters reviewed two case studies and discussed the rating scale for each one. The selected rater clarified any points of confusion with the rubric or the sample cases. The ICC consists of single and average measures for each rubric category. The single measure refers to the reliability of one rater; whereas the average measures refer to the reliability of an average of multiple raters. The study reports both measures; however, the single measure ICC is most appropriate as usually one instructor would rate the students' case studies. For the sample, the ICC single measures and average measures for each rubric category and total score for case study one were each .905 and above. The ICC single measures and average measures for each rubric category and total score for case study two were .791 and above, and .883 and above, respectively. Interpretation of ICC values suggests that values above .75 indicate excellent agreement (Cicchetti, 1994). Table 5 lists the ICC values for each case study. 
Table 5

ICC Results for PACCI

\begin{tabular}{llll}
\hline Rubric Category & $\begin{array}{l}\text { Single vs Average } \\
\text { Measures }\end{array}$ & $\begin{array}{l}\text { Case Study 1 } \\
\text { Intraclass } \\
\text { Correlation }\end{array}$ & $\begin{array}{l}\text { Case Study 2 } \\
\text { Intraclass } \\
\text { Correlation }\end{array}$ \\
\hline Cultural Knowledge & Single Measures & $.925^{\mathrm{a}}$ & $.955^{\mathrm{a}}$ \\
& Average Measures & $.961^{\mathrm{c}}$ & $.977^{\mathrm{c}}$ \\
Cultural Awareness & Single Measures & $.905^{\mathrm{a}}$ & $.791^{\mathrm{a}}$ \\
& Average Measures & $.950^{\mathrm{c}}$ & $.883^{\mathrm{c}}$ \\
Cultural Skills & Single Measures & $.957^{\mathrm{a}}$ & $.813^{\mathrm{a}}$ \\
& Average Measures & $.978^{\mathrm{c}}$ & $.897^{\mathrm{c}}$ \\
Total Score & Single Measures & $.947^{\mathrm{a}}$ & $.932^{\mathrm{a}}$ \\
& Average Measures & $.973^{\mathrm{c}}$ & $.965^{\mathrm{c}}$ \\
\hline
\end{tabular}

Two-way mixed effects model where people effects are random and measures effects are fixed.

a. The estimator is the same, whether the interaction effect is present or not.

b. Type A intraclass correlation coefficients using an absolute agreement definition.

c. This estimate is computed assuming the interaction effect is absent, because it is not estimable otherwise.

PACCS. The indirect measure included 15 competencies, comprised of the top three competencies from the 27 identified from the modified Delphi method, to reduce survey fatigue. Five additional competencies were included based on the final definition of cultural competence from the Delphi.

The competencies identified as part of the Delphi and used for the PACCS help to establish validity of the assessment. The internal consistency of the 15 items used for testing is .940 . The overall internal consistency of the trimmed 3-factor scale ( 8 items) used in the data analysis phase is .887 . The scale included in the survey consists of 15 items, listed below: 
1. I am able to identify my own underlying biases, perspectives, and values.

2. I recognize that cultural competency development requires continuous learning and I am committed to this process.

3. I am aware of how my culture impacts communication.

4. I can apply strategies to mitigate communication barriers.

5. I appreciate diversity and am sensitive to the differences between cultures.

6. I can define cultural competence and concepts related to cultural competence such as social justice, social equity, and diversity.

7. I am able to explain the importance of cultural competency in the policymaking process.

8. I understand how to develop culturally competent policies.

9. I can apply cultural awareness and knowledge to communicate effectively within various domestic and international contexts.

10. I can function effectively within a diverse team.

11. I can communicate effectively with a diverse citizenry.

12. I can communicate effectively with diverse coworkers.

13. I can proficiently and openly discuss issues related to cultural competency. 14. I can assess outcomes to identify cultural disparities.

15. I can implement appropriate cultural interventions.

Operationalization of independent variables. There are four main independent

variables in this study: PSM, MPA Exposure to Diversity, CoBRAS, and Lifetime Experience with Discrimination.

PSM. Perry (1996) developed the first public service motivation scale, which consisted of 40-items. After testing for reliability and validity, Perry reduced the scale to 24-items; this scale produced an overall Cronbach's alpha of .9 (Perry, 1996). Due to the length of the scale, several authors have used a shortened version of the scale, consisting of 5 items, using a 7-point Likert scale (Alonso \& Lewis, 2001; Christensen \& Wright, 2010; Pandey, Wright, \& Moynihan, 2008). The items chosen for this scale "capture three dimensions - commitment to public interest, compassion and self-sacrifice identified by Perry (1996) that represent the affective or normative motives most closely associated with the altruistic appeal of public sector values" (Pandey, Wright, \& Moynihan, 2008, p. 15). Alonso \& Lewis (2001) found the Cronbach's alpha of the 5- 
item scale to be 7 with a sample size of more than 7000; Christensen \& Wright (2010), reported a Cronbach's alpha of .77, with a sample size of 182; Pandey, Wright, \& Moynihan (2008) reported a Cronbach's alpha of .74, with a sample size of 173. Per Nunnally \& Bernstein (1994), values above .7 represent a sufficient level of reliability. The Cronbach's alpha for the sample in the current study is .787. Five items make up this scale, listed below:

1. Meaningful public service is very important to me.

2. I am often reminded by daily events about how dependent we are on one another.

3. Making a difference in society means more to me than personal achievements.

4. I am prepared to make enormous sacrifices for the good of society.

5. I am not afraid to go to bat for the rights of others even if it means I will be ridiculed.

(Christensen \& Wright, 2010)

MPA exposure to diversity. This variable is not based on a published scale. It was created to understand the level of exposure that MPA students had to diversity/cultural competence issues within their MPA program. The index score is based on three questions related to course content and training within their program. Students responded to the questions using a 5-point Likert scale. The Cronbach's alpha for this study is .706. Three questions measure this concept, listed below:

1. How many courses have you taken in your MPA program that emphasize culture, diversity, race or ethnicity?

2. How much does your MPA program emphasize cultural content in required courses?

3. How many workshops and/or trainings, related to your MPA program, have you received related to cultural competence (not including courses)?

Color-blind racial attitudes scale (CoBRAS). Neville et al. (2000), developed the CoBRAS scale to measure color-blind racial attitudes. The original scale consisted of 26 items, and preliminary analysis indicated that a 20 -item scale had a better factor structure. 
Students rate the items using a 6 point Likert scale (from 1=strongly disagree to $6=$ strongly agree). The researchers tested this 20 -item scale on 594 college students and community members. The confirmatory factor analysis (CFA) demonstrated adequate fit on 3 factors. Concurrent, discriminant, and criterion-related validity demonstrated adequate scale constructions. The authors performed test-retest reliability on 102 college students; the overall reliability was .68 for the entire scale, with .8 for 2 subscales and .34 for the third subscale. The low test-retest reliability for the third subscale was attributed to training that students received in between testing. The Cronbach's alpha was .86 for the entire CoBRAS scale. Additional studies have confirmed Cronbach's alpha scores above .8 (Awad et al., 2005; Neville, Spanierman, and Doan, 2006; Chao et al., 2010). For this study the scale was operationalized as a single index to compare cultural competence to overall color-blind racial attitudes, this is in line with previous studies related to CoBRAS and Multicultural Counseling Competencies (Neville, Spanierman, \& Doan, 2006). The Cronbach's alpha of the full scale for the current study is .905. The CoBRAS scale consists of 20 items, listed below.

1. Everyone who works hard, no matter what race they are, has an equal chance to become rich.

2. Race plays a major role in the type of social services (such as type of healthcare or daycare) that people receive in the U.S.

3. It is important that people begin to think of themselves as American and not African American, Mexican American or Italian American.

4. Due to racial discrimination, programs such as affirmative action are necessary to help create equality.

5. Racism is a major problem in the U.S.

6. Race is very important in determining who is successful and who is not.

7. Racism may have been a problem in the past, but it is not an important problem today.

8. Racial and ethnic minorities do not have the same opportunities as White people in the U.S.

9. White people in the U.S. are discriminated against because of the color their skin. 
10. Talking about racial issues causes unnecessary tension.

11. It is important for political leaders to talk about racism to help work through or solve society's problems.

12. White people in the U.S. have certain advantages because of the color of their skin.

13. Immigrants should try to fit into the culture and adopt the values of the U.S.

14. English should be the only official language in the U.S.

15. White people are more to blame for racial discrimination in the U.S. than racial and ethnic minorities

16. Social policies, such as affirmative action, discriminate unfairly against White people.

17. It is important for public schools to teach about the history and contributions of racial and ethnic minorities.

18. Racial and ethnic minorities in the U.S. have certain advantages because of the color of their skin.

19. Racial problems in the U.S. are rare, isolated situations.

20. Race plays an important role in who gets sent to prison.

(Neville et al., 2000)

Lifetime Experience with Discrimination. This variable is operationalized through a single question that asked students: In your lifetime, how often have you experienced discrimination acted against you? Students responded to this question with a Likert scale with 5 options: Never; 1-3 times; 4-6 times; 7-10 times; Often: Over 10 times.

Operationalization of control variables. In addition to the dependent and independent variables presented above, it is important to describe the variables that the literature has identified as influencing cultural competence. Student level control variables are important to include, as the literature demonstrates that individual's characteristics and experiences gained outside of education or professional training influence levels of cultural competence (Allison et al., 1996; Benkert et al., 2011; Chee et al., 2012; Kohli, 2010; Reimann et al., 2004). 
Each of the control variables selected were significant in previous studies related indirect cultural competence assessment results. Of primary importance is social desirability bias. Per Wiggins (1959), "social desirability response style may be defined as a general tendency to endorse personality inventory items that are judged to be socially acceptable by people in general" (p. 419). Research related to social desirability bias and its relationship with cultural competence shows mixed results. Constantine (2000); Sodowsky et al. (1994); and Ponterotto et al (1996) found either no correlation, or insignificant correlations between social desirability and indirect multicultural competency assessments. Whereas, Constantine \& Ladany (2000); Reyes, Hadley, and Davenport (2013); Bernhard et al. (2015); Worthington, Mobley, \& Tan (2000); and Sodowsky et al (1998) did find correlations between social desirability and responses on indirect multicultural competency assessments. Since social desirability response bias depends on how transparent the self-report measures are to the person taking the assessment (Constantine, 2000), it is important to understand how it is related to the direct and indirect instruments developed for this dissertation. Furthermore, including social desirability bias response scales remains an important best practice for cultural competency research (Benkert et al., 2011).

The survey included student level characteristics along with the scale information to measure the independent variables. Table 6 includes the operationalization of the control variables. 
Table 6

Operationalization of Student-Level Control Variables

\begin{tabular}{|c|c|}
\hline Control Variables & \\
\hline $\begin{array}{l}\text { Social Desirability (Constantine \& } \\
\text { Ladany, 2000; Reyes, Hadley, and } \\
\text { Davenport, 2013; Bernhard et al. 2015; } \\
\text { Worthington, Mobley, \& Tan, 2000; } \\
\text { and Sodowsky et al,1998) }\end{array}$ & $\begin{array}{l}\text { 5-item scale operationalized as an index. } 1= \\
\text { lower social desirability bias; } 5=\text { higher } \\
\text { social desirability bias (Hays, Hayashi, \& } \\
\text { Stewart, 1989). Scale items are listed below. } \\
\text { 1. I am always courteous even to people } \\
\text { who are disagreeable. } \\
\text { 2. There have been occasions when I } \\
\text { took advantage of someone. } \\
\text { 3. I sometimes try to get even rather } \\
\text { than forgive and forget. } \\
\text { 4. I sometimes feel resentful when I } \\
\text { don't get my way. } \\
\text { 5. No matter who I'm talking to, I'm } \\
\text { always a good listener. }\end{array}$ \\
\hline $\begin{array}{l}\text { Number of languages spoken }(\mathrm{Ng} \mathrm{\&} \\
\text { Earley, 2006; Chae et al., 2012; } \\
\text { Reimann et al., 2004) }\end{array}$ & $\begin{array}{l}\text { Operationalized as dummy variables, with } \\
\text { one language as the control group. } \\
\text { Number of languages spoken with } \\
\text { professional proficiency or higher: } \\
\text { 1. } 1 \\
\text { 2. } 2 \text { or more }\end{array}$ \\
\hline $\begin{array}{l}\text { Gender (Ng \& Earley, 2006; Kohli, } \\
\text { 2010; Benkert et al., 2011) }\end{array}$ & $\begin{array}{l}\text { Operationalized as dummy variables, with } \\
\text { female as the control group. } \\
\text { Gender Identity: } \\
\text { 1. Man } \\
\text { 2. Woman }\end{array}$ \\
\hline $\begin{array}{l}\text { Race/Ethnicity (Kohli, 2010; Reimann } \\
\text { et al, 2004; Benkert et al., 2011) }\end{array}$ & $\begin{array}{l}\text { Operationalized as dummy variables, with } \\
\text { White as the comparison group. } \\
\text { To which racial group do you most } \\
\text { identify? } \\
\text { 1. White } \\
\text { 2. Black or African American } \\
\text { 3. Asian } \\
\text { 4. Race not listed }\end{array}$ \\
\hline $\begin{array}{l}\text { Lived or Studied Abroad (Koskinen \& } \\
\text { Tossavainen, 2004; Ruddock \& Turner, } \\
\text { 2007; Kitsantas, 2004). }\end{array}$ & $\begin{array}{l}\text { Ordinal Variable. } \\
\text { Have you lived or studied abroad? }\end{array}$ \\
\hline
\end{tabular}




\begin{tabular}{|l|ll|}
\hline & 1. & No \\
& 2. & One semester \\
& 3. & One year \\
& 4. & More than one year \\
\hline Age (Benkert et al., 2011; Kohli et al., & Ordinal Variable \\
2010; Ng \& Earley, 2006)) & Age & \\
& 1. & $18-24$ \\
& 2. & $25-34$ \\
& 3. & $25-44$ \\
& 4. & $45-54$ \\
& 5. & $55-64$ \\
& 6. & 65 and older \\
\hline
\end{tabular}

\section{Data Analysis}

The researcher used five programs to perform analysis in this study: NVivo 10 to code and analyze the qualitative data for the Delphi survey; STATA 14 to generate descriptive statistics; Microsoft Excel 2016 to organize the data; and AMOS 23 to conduct confirmatory factor analyses. STATA 14 was then used to perform OLS regression in phase 2 . The data analyses completed for each phase are outlined below.

The Delphi results were analyzed and coded according to theme with STATA 14 and NVivo 10. To determine consensus for the first round, competencies with less than $51 \%$ consensus for scale point 9 (strongly agree) was removed from the list (Loughlin \& Moore, 1979). The cultural competence definition was edited based on the qualitative feedback. For round two, new competencies were added based on participant feedback. After round two was complete, any competency with less than $51 \%$ consensus for scale point nine (strongly agree) was be removed from the list. Round three consisted of the final definition and competencies for the panel to review. The panelists provided no further feedback and the definition and competencies were finalized. 
Data analysis for Phase 1B included construction of the dependent and independent variables through confirmatory factor analysis. The results of the CFA of the PACCS and PACCI answered research question 1c. The purpose of CFA is to gain information about variables not directly observed. The unobserved variable is a latent variable, and the variables used to measure this latent variable are observed or manifest variables (Schreiber et al., 2006). CFA is also different from other analyses in the sense that it is theory-driven, and "requires the investigator to specify both the number of factors and the specific pattern of loadings of each of the measured variables on the underlying set of factors" (Curran, West, \& Finch, 1996, p. 16).

Data analysis for the PACCI was conducted on 234 completed case studies. AMOS 23 was used to test the hypothesized two-factor model and a one-factor model via confirmatory factor analysis. Data analysis for the PACCS was conducted on 252 completed surveys. Using AMOS 23, a three-factor model, a trimmed three-actor model, and a one-factor model were tested using CFA.

Phase 2 consisted of examining the relationships between the dependent and independent variables in the study. The researcher coded the data and performed the OLS regressions analyses with STAT14. OLS requires that the data meet seven assumptions; in this study, that data violated the assumption of normally distributed errors and constant variance. For normally distributed errors, Lumley et al. (2002) found that for large samples, valid estimates can be calculated from non-normal distributions. While the definition of a large sample is not equivalent in all scenarios, "previous simulation studies show that 'sufficiently large' is often under 100" (Lumley et al., 2002, p.166). While the sample in this study exceeds 100, a cubic transformation of the PACCS dependent 
variable was used to verify results. To account for the presence of heteroscedasticity, robust standard errors were employed. Furthermore, each regression uses fixed effects to control for the correlation between data from each of the 14 sampled schools.

\section{Summary}

The study utilized a sequential two-phased mixed methods design. The first phase of the study focused on developing a direct and indirect measure of public administrator cultural competence. Phase 1A addressed the identification of a discipline-specific cultural competence definition for public administration, as well as of the related competencies that public administrators should be able to demonstrate. Once this definition and the related competencies were identified, the dependent variables (the PACCI and the PACCS), were constructed. Phase 1B of the study consisted of the data collection process and then use of CFA to validate the PACCI and PACCS. In Phase 2 of the study, regression analyses were performed to examine the relationship between the three independent variables (CoBRAS, PSM, Lifetime Experience with Discrimination, and Exposure to Diversity) and the dependent variables (PACCI, PACCS). 


\section{CHAPTER 5}

\section{MEASUREMENT OF CULTURAL COMPETENCE IN PUBLIC}

\section{ADMINISTRATION}

This chapter of the dissertation discusses the results of Phase 1 of the study. The chapter contains two sections: Phase 1A results and Phase 1B results. The Delphi method performed during Phase 1A resulted in a definition of cultural competence for public administration, and identification of 27 competencies that are important for public administrators to demonstrate. The confirmatory factor analyses (CFA) performed during Phase 1B resulted in a two-factor solution for the PACCI; and a trimmed three factor solution for the PACCS.

\section{Phase 1A: Modified Delphi method}

The purpose of Phase 1A was to answer research question 1:

1a: How is cultural competence defined in public administration?

1b: What cultural competencies should public administrators be able to demonstrate?

The results of the modified Delphi method answered each of the above research questions. After three rounds, the panel of experts reached a consensus for the definition of cultural competence, and the cultural competencies required of public administrators.

\section{Definition of Cultural Competence for Public Administration}

Based on the analysis of the Delphi results, the definition of cultural competence for public administration is:

...the set of attitudes, knowledge, and skills that are required for an organization or individual to effectively serve the public sector and navigate the cultural 
dimensions of an organization. A culturally competent public organization is one that incorporates cultural competence into policy making, public service delivery, and the internal organizational infrastructure, culture, and norms.

A culturally competent public administrator:

1. Articulates the value of cultural competence and recognizes that cultural competence requires lifelong learning;

2. Engages in self-assessment of their cultural awareness, knowledge, and skills;

3. Appreciates diversity and is sensitive to the differences between cultures;

4. Communicates effectively with a diverse citizenry and workforce and adapts services to meet their needs;

5. Is able to contribute to the institutionalization of cultural knowledge; promote cultural competence; identify cultural disparities; and monitor cultural competence performance. (Rice, 2007; Cross et al., 1989; Denboba, 1993)

\section{Cultural Competencies Required of Public Administrators}

The Delphi identified 27 competencies. Table 7, 8, and 9 outline each of these awareness, knowledge, and skills competencies, as well as related descriptive statistics, respectively. Each of the retained competencies had a mean of above 8, a median score of 9 , and an interquartile range no greater than 2 . 


\section{Table 7}

Delphi Results: Cultural Awareness Descriptive Statistics

$\begin{array}{llll}\text { A culturally aware public } & \text { n } & \text { Mean } & \begin{array}{l}\text { Standard } \\ \text { Deviation } \\ \text { administrator... }\end{array}\end{array}$

$\begin{array}{llllll}\text { Reflects on one's self-identity and } & 15 & 8.93 & 0.258 & 9 & 0\end{array}$

identifies underlying biases, perspectives, and values.

Recognizes that cultural

$14 \quad 8.79 \quad 0.579$

9

0

competency development requires

continuous learning, and is

committed to this process.

Displays awareness of how one's

$\begin{array}{lll}15 & 8.73 \quad 0.594\end{array}$

9

0

culture impacts communication

and can apply strategies to

mitigate communication barriers.

Demonstrates a commitment to

$15 \quad 8.67$

0.9

9

0

creating an inclusive workplace.

Respects those with different

15

8.6

0.632

9

1

cultural perspectives and

demonstrates empathy.

Upholds a commitment to social

15

8.47

0.743

9

1

equity, social justice, and social

change.

Exhibits a willingness to adapt

$\begin{array}{lll}15 & 8.47 & 0.915\end{array}$

9

1

services or find appropriate

resources to overcome cultural

barriers that may arise.

Recognizes how power is exerted

$\begin{array}{lll}15 & 8.27 & 1.387\end{array}$

9

1

in social, political, administrative,

economic, and legal settings.

Demonstrates openness to change

15

8.2

1.373

9

1

their own perspectives and

understand that change is

important and valuable. 
Recognizes the existence of both

$\begin{array}{lll}15 & 8.07 \quad 1.486\end{array}$

9

invisible and visible dimensions of

diversity.

(Rice and Mathews, 2012; Carrizales, 2010; Tilford Group, 2001)

\section{Table 8}

Delphi Results: Cultural Knowledge Descriptive Statistics

\begin{tabular}{|c|c|c|c|c|c|}
\hline $\begin{array}{l}\text { A culturally knowledgeable } \\
\text { public administrator... }\end{array}$ & $\mathbf{N}$ & Mean & $\begin{array}{l}\text { Standard } \\
\text { Deviation }\end{array}$ & Median & $\begin{array}{l}\text { Interquartile } \\
\text { Range }\end{array}$ \\
\hline $\begin{array}{l}\text { Demonstrates a normative } \\
\text { understanding of cultural } \\
\text { competence and concepts } \\
\text { related to cultural competence, } \\
\text { including: social justice, social } \\
\text { equity, and diversity. }\end{array}$ & 14 & 8.64 & 0.497 & 9 & 1 \\
\hline $\begin{array}{l}\text { Describes the importance of } \\
\text { cultural competence in policy } \\
\text { development and explains how } \\
\text { to include cultural competency } \\
\text { in the policy-making process. }\end{array}$ & 14 & 8.29 & 1.326 & 9 & 1.25 \\
\hline $\begin{array}{l}\text { Demonstrates how culture } \\
\text { frames, informs, and shapes } \\
\text { policy. }\end{array}$ & 14 & 8.29 & 1.383 & 9 & 1 \\
\hline $\begin{array}{l}\text { Understands the cultural } \\
\text { competence continuum and } \\
\text { identifies the competencies } \\
\text { required to move along the } \\
\text { continuum. }\end{array}$ & 14 & 8.29 & 1.49 & 9 & 1 \\
\hline $\begin{array}{l}\text { Describes socio-, political, } \\
\text { economic, and historic } \\
\text { frameworks that have } \\
\text { contributed to the oppression } \\
\text { of groups within their } \\
\text { community. }\end{array}$ & 14 & 8.21 & 1.424 & 9 & 1.25 \\
\hline
\end{tabular}




\title{
Table 9
}

Delphi Results: Cultural Skills Descriptive Statistics

$\begin{array}{cccccc}\begin{array}{c}\text { A culturally skilled public } \\ \text { administrator... }\end{array} & \text { N } & \text { Mean } & \begin{array}{c}\text { Standard } \\ \text { Deviation }\end{array} & \text { Median } & \begin{array}{c}\text { Interquartile } \\ \text { Range }\end{array}\end{array}$

\begin{abstract}
Applies cultural awareness and
knowledge to communicate

effectively within various

domestic and international

cultural contexts.
\end{abstract}

8.79

0.579

9

0

Functions effectively within

14

diverse team.

$\begin{array}{lll}14 & 8.79 & 0.579\end{array}$

9

0

Communicates effectively with

$14 \quad 8.79$

0.579

9

0

a diverse citizenry and

workforce.

Engages in organizational and

14

8.5

1.092

9

1

self- assessment to improve

cultural competency outcomes.

Proficiently and openly

8.5

1.092

9

1

discusses issues related to

cultural competency to

contribute to the

institutionalization of cultural

knowledge within the

organization.

Assesses outcomes to identify

cultural disparities.

Identifies and eliminates

barriers to participation and

equity.

Conducts a needs assessment

$14 \quad 8.36$

1.151

9

1

14

14

8.43

0.938

9

0.25 services that are appropriate for their community. 
Implements effective strategies

$14 \quad 8.29$

1.204

9

0

to motivate culturally different

individuals and groups.

Implements appropriate

14

8.21

1.188

9

1.25

cultural interventions.

Constructs equitable solutions

14

8.14

1.292

9

1.25

for protracted social, political,

administrative, economic, and

legal problems.

Creates support structures for

$14 \quad 8.07$

1.141

8

1.25

individuals, groups, and

organizations that face limited

or skewed participation and

inequity.

(Rice and Mathews, 2012; Carrizales, 2010; Tilford Group, 2001)

Each of the retained items map onto the conceptual framework developed in

Chapter 3. These competencies guided development of the PACCS and PACCI instruments.

\section{Phase 1B: Development of the PACCI and PACCS}

Data analysis for the PACCI (direct measure) and PACCS (indirect measure) was performed in AMOS 23 via CFA. The latent variable of interest in this study is cultural competence; whereas the observed variables are the PACCI and PACCS items, developed from the results of the modified Delphi method in phase 1A. To determine whether the specified model is appropriate, fit indices must be examined. Fit indices fall into two categories, either absolute fit or incremental fit (Hu \& Bentler, 1999). According to $\mathrm{Hu} \&$ Bentler, the absolute fit index is used to "assess how well an a priori model reproduces the sample data", whereas the incremental fit index "measures the proportional improvement in fit by comparing a target model with a more restricted, 
nested base model" (p. 2). There are several fit indices that exist to gauge model fit; for this study uses: the chi-square $\left(\chi^{2}\right)$ statistic, the Comparative Fit Index (CFI), Tucker Lewis Index (TLI), Root Mean Square Error of Approximation (RMSEA), $p$ of Close Fit (PCLOSE), and the Standardized Root Mean Square Residual (SRMR).

Traditionally, the $\chi^{2}$ statistic is the most commonly cited fit index (Gierl \& Mulvenon, 1995), and demonstrates that a model is good fitting when the statistic has a p-value of greater than .05 (Barrett, 2007). According to Hu \& Bentler (1999), while a non-significant $\chi^{2}$ value indicated model misspecification, or violations of statistical assumptions, "it has been commonly recognized that models are best regarded as approximations of reality, and hence, using chi-square to test the hypothesis that the population covariance matrix matches the model-implied covariance matrix... is too strong to be realistic" (p. 425). Furthermore, $\chi^{2}$ is sensitive to sample size, and is more likely to "fail to fit" when the sample size is large (Barrett, 2007). Due to the heavy reliance on sample size, scholars developed a different measure based on the $\chi^{2}$ statistic: relative/normed $\chi^{2}$ (Wheaton et al., 1977). This statistic is based on the $\chi^{2}$ value divided by the degrees of freedom; however, it is also subject to interpretation, with acceptable ratios ranging from 2 to 5 (Hooper, Coughlan \& Mullen, 2008). Alternative fit indices address this "restrictiveness". For the purposes of this study, models include the $\chi^{2}$ statistic, but the relative/normed $\chi^{2}$ will determine mode fit. In addition to the $\chi^{2}$ statistic, the study refers to two additional absolute fit indices, including RMSEA and SRMR.

The RMSEA describes how well the specified model fits the population's covariance matrix, and favors parsimony, "in that it will choose the model with the lesser 
number of parameters" (Hooper, Coughlan, \& Mullen, 2008, p.54). The suggested cutoff value for the RMSEA is .06 (Hu \& Bentler, 1999). Related to RMSEA is the PCLOSE statistic. This statistic is related to the confidence interval that is calculated for RMSEA, with a value greater than .05 indicating a close fit. The SRMR is the "standardized difference between the observed correlation and the predicted correlation" (Kenny, 2015). The suggested cutoff value for the SRMR is .08 (Hu \& Bentler, 1999).

The study also uses two incremental fit indices to determine goodness of fit, the CFI and TLI. The CFI ranges from 0 to 1 , with 1 indicating excellent model fit. Furthermore, sample size does not greatly influence this fit measure, and "assumes that all latent variables are uncorrelated (null/independence model) and compares the sample covariance matrix with this null model (Hooper, Coughlan, \& Mullen, 2008, p. 55). The TLI also compares the $\chi^{2}$ statistic of the null model with the $\chi^{2}$ statistic of the hypothesized model. While most TLI values fall between 0 and 1 , it is possible to have values outside of this range. The suggested cutoff value for the CFI and TLI is .95 (Hu \& Bentler, 1999).

This section of the dissertation first discusses the results of the PACCI, followed by the results for the PACCS.

\section{PACCI}

After data collection and coding, analysis of the PACCI took place. Survey data and the rubric scores for the 234 students were exported to AMOS 23 for analysis. The rubric consisted of three sub-scales (knowledge, awareness, and skills). There were 4 total points possible for each sub scale, for a rubric total of 12 for each case study. Table 10 presents the mean and standard deviation for each of the case studies. 
Table 10

PACCI Case Study Descriptive Statistics

\begin{tabular}{llllc}
\hline Variable & M & SD & Min. & Max. \\
\hline Case Study 1 - Knowledge & 2.93 & .717 & 1 & 4 \\
Case Study 1 - Awareness & 2.71 & .723 & 1 & 4 \\
Case Study 1 - Skills & 2.87 & .649 & 1 & 4 \\
\hline Case Study 2 - Knowledge & 2.80 & .988 & 1 & 4 \\
Case Study 2 - Awareness & 2.57 & .906 & 1 & 4 \\
Case Study 2 - Skills & 2.19 & .634 & 1 & 4 \\
\hline
\end{tabular}

To place the descriptive statistics into context, tables 11 and 12 give an example of actual student responses aligning to both low and high rubric scores for each subscale.

\section{Table 11}

Case Study 1 Example Answers

\begin{tabular}{|l|l|l|}
\hline Subscale & $\begin{array}{l}\text { Case Study 1 Example - Low } \\
\text { Score }\end{array}$ & $\begin{array}{l}\text { Case Study 1 Example - } \\
\text { High Score }\end{array}$ \\
\hline $\begin{array}{l}\text { Knowledge: What is the } \\
\text { conflict in this } \\
\text { situation? (1-2 } \\
\text { Sentences) }\end{array}$ & $\begin{array}{l}\text { The conflict is between } \\
\text { workers and clients }\end{array}$ & $\begin{array}{l}\text { The conflict in this situation } \\
\text { is cultural; coworkers do } \\
\text { not understand the nuances } \\
\text { of working with the new } \\
\text { population }\end{array}$ \\
\hline $\begin{array}{l}\text { Awareness: What } \\
\text { individual and } \\
\text { organizational } \\
\text { characteristics attributed } \\
\text { to this conflict? (1-2 } \\
\text { Sentences) }\end{array}$ & $\begin{array}{l}\text { Based on the current } \\
\text { information, I am uncertain } \\
\text { organizational characteristics } \\
\text { attributed to this situation }\end{array}$ & $\begin{array}{l}\text { Bias against the other and a } \\
\text { focus on efficiency of time } \\
\text { in dealing with clients. }\end{array}$ \\
\hline
\end{tabular}




\begin{tabular}{|l|l|l|}
\hline $\begin{array}{l}\text { Skills: How can this } \\
\text { conflict be resolved at } \\
\text { both the employee and } \\
\text { organizational level? (1- } \\
2 \text { Sentences) }\end{array}$ & $\begin{array}{l}\text { Training may resolve the } \\
\text { conflict for both. }\end{array}$ & $\begin{array}{l}\text { This conflict can be solved } \\
\text { by augmenting employee } \\
\text { skills in cross-cultural } \\
\text { communication, and/or } \\
\text { hiring special staff as } \\
\text { translations for clients. The } \\
\text { organization should } \\
\text { implement an inter-cultural } \\
\text { competency training. }\end{array}$ \\
& & \\
\hline
\end{tabular}

For case study 1 , the low scores demonstrate the inability to identify the cultural conflict; whereas the answer that received a high score accurately identified the presence of a cultural conflict. For awareness, the low score answer did not provide any details, and was not able to identify contributing factors; the answer receiving a higher score identified the bias on the part of the employees, and the unpreparedness of the organization to welcome a new clientele. For cultural skills, the low scoring answer provided one means of solving the conflict; while the high scoring answer mentioned three ways to resolve the conflict, not only through two types of training, but also through hiring additional staff members.

As evidenced by the descriptive statistics, students answering case study 1 scored highest on the knowledge sub-score, and lowest on the awareness sub-score. Overall, students had difficulty identifying both organization and employee level attributes that contributed to the situation. 
Table 12

Case Study 2 Example Answers

\begin{tabular}{|l|l|l|}
\hline & $\begin{array}{l}\text { Case Study 2 Example - Low } \\
\text { Score }\end{array}$ & $\begin{array}{l}\text { Case Study 2 Example - } \\
\text { High Score }\end{array}$ \\
\hline $\begin{array}{l}\text { Knowledge: What is the } \\
\text { conflict in this } \\
\text { situation? (1-2 } \\
\text { Sentences) }\end{array}$ & $\begin{array}{l}\text { Husband is angry for being } \\
\text { told what to do }\end{array}$ & $\begin{array}{l}\text { The cultural customs are } \\
\text { different between } \\
\text { admissions officer and } \\
\text { couple. The admissions } \\
\text { officer was presumptuous } \\
\text { and offensive, insensitive. } \\
\text { Conflicting cultural values. }\end{array}$ \\
\hline $\begin{array}{l}\text { Awareness: What } \\
\text { individual and } \\
\text { organizational } \\
\text { characteristics attributed } \\
\text { to this conflict? (1-2 } \\
\begin{array}{l}\text { Sentences) } \\
\text { hention }\end{array}\end{array}$ & $\begin{array}{l}\text { Husband was speaking for } \\
\text { his wife }\end{array}$ & $\begin{array}{l}\text { The officer's assumption } \\
\text { that a man who answers for } \\
\text { his wife is controlling. The } \\
\text { American ideal of female } \\
\text { independence and how this } \\
\text { cultural value has shaped } \\
\text { each party's perspective. }\end{array}$ \\
\hline $\begin{array}{l}\text { Skills: How can this } \\
\text { conflict be resolved at } \\
\text { both the employee and } \\
\text { organizational level? } \\
\text { 2 Sentences) }\end{array}$ & $\begin{array}{l}\text { Require a one-on-one } \\
\text { meeting, not allowing } \\
\text { husband to join }\end{array}$ & $\begin{array}{l}\text { Apologize for the reaction, } \\
\text { admit lack of sensitivity. } \\
\text { Educate admissions officer } \\
\text { about new cultures; } \\
\text { encourage to learn. }\end{array}$ \\
\hline
\end{tabular}

For case study two, the low scores demonstrated that students focused on the husband's attitude and behavior as the source of the conflict and the characteristics that contributed to the problem. Whereas the higher scoring answers accurately identified the cultural conflict and could identify the biases that the admissions officer had toward the couple's communication style. For the skill sub-score, the lower scoring answer did not address a means for the employee to improve their cultural competence skills, instead focusing on the husband as the root of the problem; the higher scoring answer addressed 
the need to take responsibility, and highlighted the importance of education for improving future interactions.

As evidenced by the descriptive statistics, students answering case study 2 scored highest on the knowledge sub-score, and lowest on the skills sub-score. For this case study, students had the most difficulty with identifying how to resolve the conflict.

The case study scores were lower overall for case study 2 ; since the underlying cultural issues were more nuanced. The discussion of "invisible" cultural characteristics challenge students in their interpretation of the scenario; whereas the first case study dealt with a simpler problem that was more overtly stated in the prompt.

Due to the nature of the case study questions, it was hypothesized that each question would load onto a separate latent variable. This is because the first question measured lower-order cultural competence skills; while the second question measured higher-order cultural competence skills. AMOS 23 was used to test the hypothesized twofactor model and a one-factor model. The two-factor model provided the best fit. Factor scores for each of the case studies were exported into STATA, followed by the generation of separate factor scores. While a one-factor, higher order model, would have streamlined the results of the study, it was not possible to conduct due to limited degrees of freedom. Table 13 outlines the fit indices for each of the tested models. When compared to the one factor model, the two-factor model demonstrates a considerably better fit, with a small $\chi^{2}$ and CMIN/DF ratio; and CFI, TLI, SRMR, RMSEA, and PCLOSE values that meet the required thresholds. Based on the model fit statistics, hypotheses 1 is confirmed. 
Table 13

PACCI CFA Fit Indices

\begin{tabular}{llc}
\hline Fit Index & Two Factor & One Factor \\
\hline$\chi^{2}$ & 11.298 & 18.494 \\
CMIN/DF & 1.412 & 2.055 \\
CFI & .974 & .925 \\
TLI & .951 & .875 \\
SRMR & .0375 & .0515 \\
RMSEA & .042 & .067 \\
PCLOSE & .536 & .224 \\
\hline
\end{tabular}

Figure 8 presents the two-factor model with standardized factor loadings.

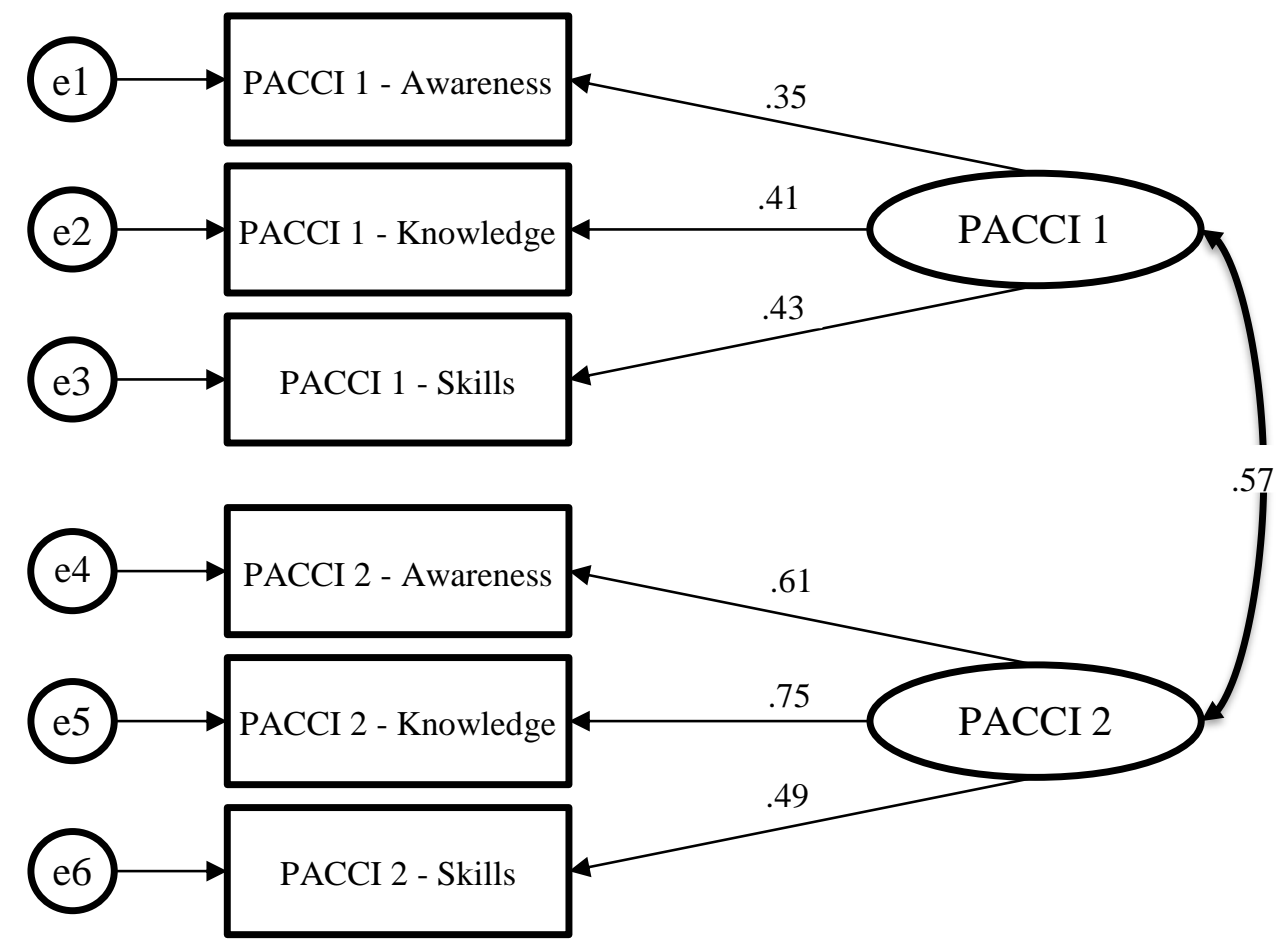

Figure 8 PACCI Standardized Factor Loadings 
While the overall fit of the CFA model for the PACCI is acceptable. It is important to discuss the parameter estimates. A conservative rule-of-thumb for factor loadings in CFA models is .7; however, .4 is acceptable as a lower threshold (Matsunaga, 2011). While five of the six factor loadings exceed this minimum threshold, Hair et al. (1995) note that .4 is an acceptable factor loading for samples of 200 and .35 is an acceptable factor loading for samples of 250. Furthermore, retention of theoretically important factors is justifiable for CFA models.

In addition to the factor scores, it is also important to examine the reliability and convergent and discriminant validity for the factors. For CFA models, composite reliability is a better measure of reliability than Cronbach's alpha; the conservative cutoff for CR is .7. For the PACCI1 factor, the CR is .359, for PACCI2 the CR is .652. Average Variance Extracted (AVE) determines the convergent reliability in CFA models, the conservative cutoff is .5. The AVE for PACCI1 is .159 and for PACCI2 is .392. While the AVE is a more conservative measure, the CR value can be used if it is close to the cutoff value of .7. A factor has discriminant validity if the Maximum Shared Variance is greater than the AVE. PACC1 does not meet this criterion; however, PACCI2 does meet this criterion. Due to the exploratory nature of this study, values that are close to these cutoffs are acceptable with the caveat that further studies need to be conducted (Ping, 2009).

Further reliability of the PACCI is demonstrated through the interrater reliability presented in Chapter 4, and face validity was tested through the review experts and the student survey pilot. Based on the goodness of fit indices, coupled with the fact that this is the first study examining a direct measure of cultural competence in public 
administration, the factors scores are deemed acceptable for use in the regression

analyses in Chapter 6.

\section{PACCS}

As described in Chapter 4, the PACCS instrument was developed from the cultural competencies identified from the Delphi study. The top three competencies from each subarea, in addition to items from the cultural competence definition were used to craft the original PACCS scale for testing. Data analysis was conducted on 252 completed surveys. The mean and standard deviation for each scale item are presented in table 14. The mean for most items in the scale were above 7 , with one competence below, indicating that students rated themselves highly on these competencies.

\section{Table 14}

\section{PACCS Descriptive Statistics}

\begin{tabular}{|c|c|c|c|c|c|}
\hline $\begin{array}{l}\text { Competency } \\
\text { Area }\end{array}$ & Scale Item & $\mathbf{M}$ & SD & Min. & Max. \\
\hline \multirow{4}{*}{$\begin{array}{c}\text { Cultural } \\
\text { Awareness }\end{array}$} & $\begin{array}{l}\text { 1. I am able to identify my own } \\
\text { underlying biases, perspectives, and } \\
\text { values. }\end{array}$ & 7.48 & 1.446 & 1 & 9 \\
\hline & $\begin{array}{l}\text { 2. I recognize that cultural competency } \\
\text { development requires continuous } \\
\text { learning and I am committed to this } \\
\text { process. }\end{array}$ & 7.90 & 1.460 & 1 & 9 \\
\hline & $\begin{array}{l}\text { 3. I am aware of how my culture impacts } \\
\text { communication. }\end{array}$ & 7.52 & 1.545 & 1 & 9 \\
\hline & $\begin{array}{l}\text { 4. I appreciate diversity and am sensitive } \\
\text { to the differences between cultures. }\end{array}$ & 7.91 & 1.391 & 1 & 9 \\
\hline $\begin{array}{c}\text { Cultural } \\
\text { Knowledge }\end{array}$ & $\begin{array}{l}\text { 1. I can define cultural competence and } \\
\text { concepts related to cultural } \\
\text { competence such as social justice, } \\
\text { social equity, and diversity. }\end{array}$ & 7.56 & 1.453 & 1 & 9 \\
\hline
\end{tabular}


2. I am able to explain the importance of $\quad \begin{array}{lllll}7.33 & 1.636 & 1 & 9\end{array}$ cultural competence in the policy-

making process.

3. I understand how to develop

$\begin{array}{llll}6.78 & 1.725 & 1 & 9\end{array}$ culturally competent policies.

1. I can apply strategies to mitigate communication barriers.

2. I can apply cultural awareness and $\quad \begin{array}{lllll}7.07 & 1.672 & 1 & 9\end{array}$ knowledge to communicate effectively within various domestic and international contexts.

3. I can function effectively within a diverse team.

Cultural

4. I can communicate effectively with a diverse citizenry.

Skills

5. I can communicate effectively with diverse coworkers.

$\begin{array}{llll}8.15 & 1.244 & 2 & 9\end{array}$

$\begin{array}{llll}7.84 & 1.378 & 2 & 9\end{array}$

$\begin{array}{llll}8.06 & 1.226 & 2 & 9\end{array}$

6. I can proficiently and openly discuss issues related to cultural competency.

7. I can assess outcomes to identify cultural disparities.

$\begin{array}{llll}7.55 & 1.464 & 1 & 9\end{array}$

8. I can implement appropriate cultural interventions.

(Rice and Mathews, 2012; Carrizales, 2010; Tilford Group, 2001)

It was hypothesized that the 15 items would load onto the assigned main construct that was identified during the Delphi. Using AMOS 23, the three-factor model indicated a poor fit; however, modification indices guided the trimming of the model based on items that co-varied with other main constructs. In addition to using modification indices to trim the model, each competency that was removed was theoretically justified; these justifications are described below. This resulted in a trimmed 
model of 8 competencies. A one-factor model was also tested to compare alternative fit structures. However, the trimmed three-factor model provided the best fit.

Table 15 contains the fit indices for each model tested. As noted below, the trimmed three factor model resulted in lower $\chi^{2}$ values and an acceptable CMIN/DF ratio. The CFI, TLI, SRMR, RMSEA, and PLCOSE also met the required thresholds for acceptable model fit.

Table 15

PACCS CFA Fit Indices

\begin{tabular}{llll}
\hline Fit Index & $\begin{array}{l}\text { Trimmed } \\
\text { Three Factor }\end{array}$ & Three Factor & One Factor \\
\hline$\chi^{2}$ & 30.434 & 719.755 & 889.260 \\
CMIN/DF & 1.790 & 8.272 & 9.881 \\
CFI & .990 & .790 & .735 \\
TLI & .983 & .747 & .691 \\
SRMR & .0219 & .0911 & .0867 \\
RMSEA & .056 & .170 & .188 \\
PCLOSE & .343 & .000 & .000 \\
\hline
\end{tabular}

The trimmed three-factor model with standardized factor loadings is presented in figure 9. 


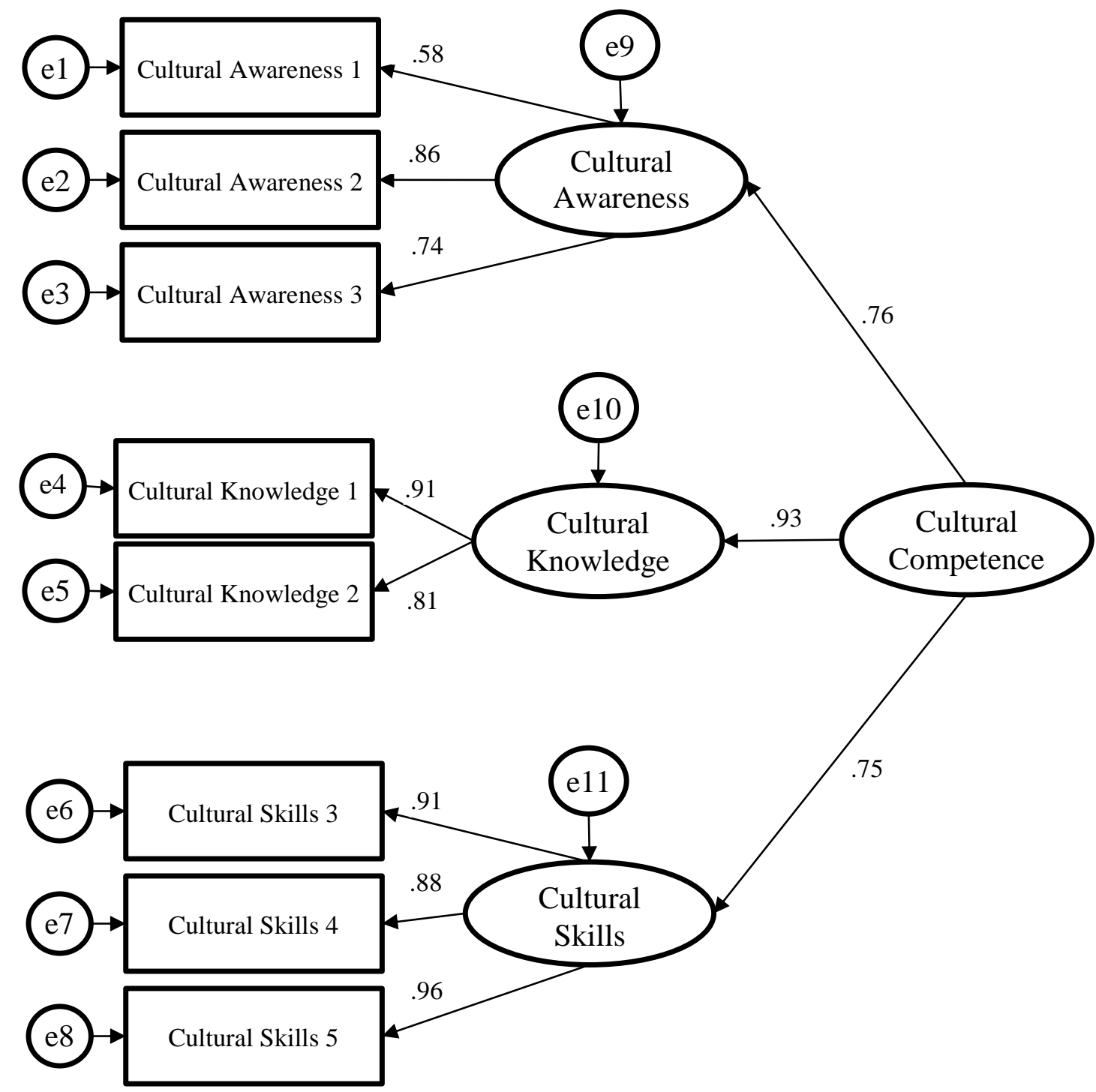

Figure 9 PACCS Standardized Factor Loadings

In addition to the goodness of fit statistics, the factor loadings for the PACCS exceed the minimum threshold of .4 , and all but one of the factor loadings meet the conservative requirement of .7. The CR and AVE values for each factor exceeds the conservative cutoffs; and the MSV values for each factor are less than the AVE. Thus, the PACCS meets tests for reliability, and convergent and discriminant validity. 
The results of the PACCS factor analysis align with the Delphi results. For cultural awareness, the three retained PACCS competencies reflected the three highest ranked competencies from the Delphi study. The awareness competency that was trimmed - I appreciate diversity and am sensitive to the differences between cultures came from the developed definition of cultural competence; this higher-order competency is reflected throughout the retained competencies.

For cultural knowledge, the two retained competencies were also the two highest rated in the Delphi study. The trimmed competency - I understand how to develop culturally competent policies - encompassed a portion of the second highest cultural knowledge competency, but was separated into its own competency to allow for more specificity. The justification to trim this competency was grounded in its similarity to the retained knowledge competencies in terms of the relationship between cultural competency and policymaking.

The cultural skills competencies underwent the largest reduction as part of the CFA. The retained competencies are all highly related to the ability to communicate effectively with a diverse citizenry and coworkers; they are also within the highest rated skills competencies from the Delphi study. The trimming of CCS1 (I can apply strategies to mitigate communication barriers), CCS2 (I can apply cultural awareness and knowledge to communicate effectively within various domestic and international contexts), and CCS6 (I can proficiently and openly discuss issues related to cultural competency) therefore are congruent, as they are all related to developing the skills that would be necessary to effectively communicate. The trimming of CCS7 (I can assess outcomes to identify cultural disparities) and CCS8 (I can implement appropriate cultural 
interventions) was due to their high correlation with the knowledge construct. Since these competencies are higher order in nature, in terms of their application, they may not be appropriate for an MPA level scale. Overall, the proposed model confirms the three hypothesized constructs and includes most of the highest rated competencies from the Delphi study.

\section{Summary}

This chapter summarized the results of the CFA for both the PACCI and the PACCS. The results of the PACCI CFA confirmed the two-factor hypothesis that each case study mapped onto a separate factor. Although there were not enough degrees of freedom to perform a higher order CFA and generate a single factor score, regression analyses were performed on both factor scores. The results of the PACCS CFA confirmed the hypothesis that a three-factor model was a good fit; however, a trimmed model was necessary to confirm good model fit. The results of each CFA analysis will be used in Chapter 6 to identify predictors of cultural competence. 


\section{CHAPTER 6}

\section{PREDICTORS OF CULTURAL COMPETENCE FOR GRADUATING MPA STUDENTS}

The purpose of this chapter is to discuss the statistical results for Phase 2 of the study. The chapter first presents the descriptive statistics for the variables. Following is a discussion of the regression results for the PACCS indirect measure. The results of this analysis confirmed the hypothesis for PSM, CoBRAS, and Lifetime Experience with Discrimination; however, there was no evidence of a significant relationship with MPA Exposure to Diversity. The final section discusses the outcomes of the PACCI direct measures. The results of this analysis confirm the hypothesis for CoBRAS and Lifetime Experience with Discrimination; however, there was no evidence of a significant relationship with PSM for the PACCIS or MPA Exposure to Diversity for either measure. In terms of the relationship between the PACCS and the PACCI, the single factor PACCS score was not a significant predictor of the PACCI score.

\section{Descriptive Statistics}

Table 16 outlines the descriptive statistics for each non-binary variable in the study. Table 17 lists the descriptive statistics coded as binary variables. In terms of dependent variables, PACCI1 and PACCI2 represent the two factor scores of the PACCI; the mean score was slightly higher for the first case study than for the second, confirming that students had a more difficult time answering the second case study. For the full PACCS scale, the mean indicates that students rated themselves high on their perceived cultural awareness, knowledge, and skills. 
The mean of the first predictor variable, PSM, indicates that most students rated themselves as highly service oriented. For MPA Exposure to Diversity, the mean indicates that students perceived their exposure to diversity within their MPA program to be below average. The mean for CoBRAS indicates that this sample of students had lower CoBRAS on average. For Lifetime Experience with Discrimination, the sample experienced on average 4-6 instances of discrimination.

The study also controls for six student-level variables: social desirability bias, time spent studying or living abroad, age, gender, race, and number of languages spoken. The mean for social desirability bias indicates that the students in the sample were more likely to answer in a socially desirable manner. The mean for the study or lived abroad variable demonstrates that the sample tended to not have lived or studied abroad. For age, the mean age bracket of the sample was 25-34; and most of the sample was female $(62.1 \%)$, white $(64.5 \%)$, and only spoke one language $(60.7 \%)$.

Table 18 lists the correlations for each variable. None of the correlations for variables within the same model were above .7 . 


\section{Table 16}

Sample Descriptive Statistics for Scale and Ordinal Items

\begin{tabular}{llllll}
\hline Variable & Obs & Mean & Std. Dev. & Min & Max \\
\hline PACCI1 & 234 & 1.207 & .189 & .559 & 1.659 \\
PACCI2 & 234 & 1.137 & .258 & .542 & 1.734 \\
PACCS & 252 & 5.860 & .880 & .972 & 6.851 \\
PSM & 264 & 5.667 & .944 & 1.2 & 7 \\
Exposure to Diversity & 258 & 2.323 & .924 & 1 & 5 \\
CoBRAS & 253 & 2.648 & .893 & 1.15 & 5.6 \\
Experience with Discrimination & 263 & 3.103 & 1.337 & 1 & 5 \\
Social Desirability Bias Scale & 261 & 3.813 & .623 & 2 & 5 \\
Studied Abroad & 262 & 1.885 & 1.185 & 1 & 4 \\
Age & 254 & 1.906 & .814 & 1 & 6
\end{tabular}

\section{Table 17}

Sample Descriptive Statistics for Binary Variables

\begin{tabular}{lll}
\hline Gender & $\mathbf{N}$ & Percent \\
Male & 99 & $37.9 \%$ \\
Female & 162 & $62.1 \%$ \\
Race & & \\
White & 165 & $64.5 \%$ \\
Black/African American & 39 & $15.2 \%$ \\
Asian & 27 & $10.5 \%$ \\
Other Race & 25 & $9.8 \%$ \\
Languages Spoken & & \\
One & 159 & $60.7 \%$ \\
Two or more & 103 & $39.3 \%$
\end{tabular}


Table 18

Correlation Matrix

\begin{tabular}{|c|c|c|c|c|c|c|c|c|c|c|c|c|c|c|c|}
\hline & 1 & 2 & 3 & 4 & 5 & 6 & 7 & 8 & 9 & 10 & 11 & 12 & 13 & 14 & 15 \\
\hline 1. PACCS & 1.000 & & & & & & & & & & & & & & \\
\hline $\begin{array}{ll}\text { 2. } & \text { PACCII }\end{array}$ & 0.051 & 1.000 & & & & & & & & & & & & & \\
\hline $\begin{array}{ll}3 . & \text { PACCI } 2\end{array}$ & 0.086 & 0.789 & 1.000 & & & & & & & & & & & & \\
\hline 4. PSM & 0.332 & 0.149 & 0.111 & 1.000 & & & & & & & & & & & \\
\hline $\begin{array}{l}\text { 5. Exposure to } \\
\text { Diversity }\end{array}$ & 0.006 & 0.002 & -0.027 & -0.004 & 1.000 & & & & & & & & & & \\
\hline 6. CoBRAS & -0.305 & -0.179 & -0.233 & -0.289 & 0.167 & 1.000 & & & & & & & & & \\
\hline $\begin{array}{l}\text { 7. Experience with } \\
\text { Discrimination }\end{array}$ & 0.167 & 0.075 & 0.082 & 0.148 & -0.071 & -0.196 & 1.000 & & & & & & & & \\
\hline $\begin{array}{ll}\text { 8. } & \text { Social Desirability } \\
\text { Bias Scale }\end{array}$ & 0.368 & 0.064 & 0.095 & 0.234 & 0.043 & -0.034 & 0.015 & 1.000 & & & & & & & \\
\hline $\begin{array}{ll}\text { 9. Two or More } \\
\text { Languages Spoken }\end{array}$ & 0.071 & -0.080 & -0.062 & 0.057 & 0.079 & -0.001 & 0.165 & -0.053 & 1.000 & & & & & & \\
\hline $\begin{array}{l}\text { 10. Studied or Lived } \\
\text { Abroad }\end{array}$ & -0.005 & 0.062 & 0.113 & 0.101 & 0.051 & -0.057 & 0.173 & 0.008 & 0.414 & 1.000 & & & & & \\
\hline 11. Age & 0.005 & 0.115 & 0.057 & 0.173 & -0.099 & 0.030 & 0.065 & 0.225 & 0.020 & 0.075 & 1.000 & & & & \\
\hline 12. Male & -0.082 & 0.007 & -0.089 & -0.082 & 0.034 & 0.170 & -0.088 & -0.248 & 0.172 & 0.032 & 0.151 & 1.000 & & & \\
\hline $\begin{array}{l}\text { 13. Black/African } \\
\text { American }\end{array}$ & 0.191 & 0.020 & -0.025 & 0.127 & -0.055 & -0.261 & 0.217 & 0.167 & -0.022 & -0.104 & 0.017 & -0.048 & 1.000 & & \\
\hline 14. Asian & -0.128 & -0.049 & 0.039 & -0.079 & -0.042 & 0.025 & 0.053 & -0.130 & 0.383 & 0.276 & -0.043 & -0.039 & -0.146 & 1.000 & \\
\hline 15. Other Race & 0.070 & -0.049 & -0.035 & 0.023 & -0.036 & -0.025 & 0.217 & 0.024 & 0.282 & 0.100 & 0.084 & 0.062 & -0.140 & -0.113 & 1.000 \\
\hline
\end{tabular}




\section{Identifying Predictors of the PACCS}

This section of the dissertation focuses on the first dependent variable of interest, the PACCS. Table 19 presents the results of the regression analysis for PACCS as the dependent variable. The results indicate that PSM, CoBRAS, Lifetime Experience with Discrimination, and social desirability bias are significant predictors of the PACCS indirect assessment score for graduating MPA students. The adjusted $\mathrm{R}^{2}$ value of the model is .248, which means that the model explains $24.8 \%$ of the variance of the PACCS measure. These results support hypotheses 3, 5, and 6. Graduating MPA students who scored higher on the PSM scale were also more likely to score higher on the PACCS measure, all other factors held equally. This also holds true for Lifetime Experience with Discrimination. For CoBRAS, students who demonstrated more color-blond racial attitudes scored lower on the PACCS measure, all other factors held equally. The model did not provide support for hypothesis 4, related to MPA Exposure to Diversity. Social desirability bias is the only control variable that is statistically significant; indicating that students who were more likely to answer in a socially desirable manner also scored higher on the PACCS measure. 


\section{Table 19}

Estimation Results. DV: PACCS full scale

\begin{tabular}{lll}
\hline Variables & PACCS Full Scale & Std. Beta \\
\hline Constant & $3.232(.860)^{* * *}$ & .240 \\
PSM & $.234(.109)^{* *}$ & \\
Exposure to Diversity & $.058(.057)$ & -.199 \\
CoBRAS & $-.197(.074)^{* * *}$ & .124 \\
Lifetime Experience with & $.084(.039)^{* *}$ \\
Discrimination & $.460(.108)^{* * *}$ & .320 \\
Social Desirability Bias Scale & $.100(.158)$ & \\
Two or More Languages Spoken & $-.014(.058)$ & \\
Lived or Studied Abroad & $-.107(.065)$ & \\
Age & $.144(.137)$ & \\
Male & $-.007(.170)$ & \\
Black/African American & $-.264(.271)$ & \\
Asian & $.050(.243)$ & \\
Other Race & 220 & \\
\hline Observations & .334 & \\
R Squared & .248 & \\
Adj. R Squared & & \\
F & & \\
\hline Note: School fixed-effects employed & robust standard error in parentheses; ${ }^{*} \mathrm{p}<0.10 ;$ \\
\hline
\end{tabular}

\section{Identifying Predictors of the PACCI}

This section of the dissertation focuses on the second dependent variable of interest, the PACCI. Table 20 presents the results of the regression analysis for the first measure of the PACCI as the dependent variable. The results indicate that CoBRAS, 
Lifetime Experience with Discrimination, and African American/Black race are significant predictors of the first measure of the PACCI for graduating MPA students. The adjusted $\mathrm{R}^{2}$ value of the model is .087 , which means that the model explains $8.7 \%$ of the variance of the PACCI's first measure. These results support hypotheses 5, and 6. Graduating MPA students who scored higher on the Lifetime Experience with Discrimination scale were also more likely to score higher on the PACCS measure, all other factors held equally. For CoBRAS, students who demonstrated more color-blind racial attitudes scored lower on the PACCI's first measure, all other factors held equally. The model did not provide support for hypothesis 3 or 4, related to PSM and MPA Exposure to Diversity. In terms of control variables, students who reported their race of African American/Black scored lower on the PACCI's first measure when compared to students who reported their race as White.

The results for PACCI2 indicate that CoBRAS, Lifetime Experience with Discrimination, and African American/Black race are significant predictors of the second measure of the PACCI for graduating MPA students. The adjusted $\mathrm{R}^{2}$ value of the model is .108 , which means that the model explains $10.8 \%$ of the variance of the PACCI's second measure. These results support the same hypotheses as PACCI1. As noted in Chapter 1 , one of the limitations of the study is the inability to generate a single higher order PACCI factor. While this would have been preferable to streamline the results, the findings indicate that both factors are significantly related to the same predictor variables. These results will help to facilitate the discussion in Chapter 7. 
Table 20

Estimation Result. DV: PACCI Case Studies

\begin{tabular}{l|ll|ll}
\hline Variables & PACCI1 & $\begin{array}{l}\text { Std. } \\
\text { Beta }\end{array}$ & PACCI2 & $\begin{array}{c}\text { Std. } \\
\text { Beta }\end{array}$ \\
\hline Constant & $1.03^{* * *}(.228)$ & & $.960^{* * *}(.230)$ & \\
PSM & $.026(.018)$ & & $.021(.022)$ & \\
Exposure to Diversity & $.019(.017)$ & & $.013(.022)$ & \\
CoBRAS & $-.040^{* *}(.018)$ & -.193 & $-.072^{* * *}(.024)$ & -.250 \\
Experience with Discrimination & $.019^{*}(.011)$ & .136 & $.028^{* *}(.014)$ & .144 \\
Social Desirability Bias Scale & $-.002(.023)$ & & $.020(.035)$ & \\
Two or More Languages Spoken & $.018(.039)$ & & $.017(.047)$ & \\
Lived or Studied Abroad & $.007(.013)$ & & $.013(.017)$ & $.013(.029)$ \\
Age & $.026(.018)$ & & $-.016(.042)$ & \\
Male & $.009(.031)$ & & $-.133^{* *}(.055)$ & -.187 \\
Black/African American & $-.081^{* *}(.038)$ & -.155 & $.047(.064)$ & \\
Asian & $-.024(.052)$ & & $-.041(.072)$ & \\
Other Race & $-.047(.047)$ & & 198 & \\
\hline Observations & 198 & & .2209 & \\
R Squared & .2029 & & $2.48 * * *$ & \\
Adj. R Squared & .087 & & \\
F & $2.23^{* * *}$ & & \\
\hline N & & & & \\
\hline
\end{tabular}

Note: School fixed-effects employed; robust standard error in parentheses; ${ }^{*} \mathrm{p}<0.10$; $* *$ $\mathrm{p}<0.05 ; * * * \mathrm{p}<0.01$

In addition to identifying the predictors of both PACCI measures, it is also important to explore whether the PACCS measure predicts scores on the PACCI measures. For each model, multicollinearity statistics were reviewed, and no issues were found. Table 21 presents the regression results for the both PACCI measures and the full PACCS scale as a dependent variable. For this model, the full PACCS scale is not a 
significant predictor of the first PACCI measure, holding all things equally. The adjusted $\mathrm{R}^{2}$ value for this model is .081. For the second PACCI measure the full PACCS scale is not a significant predictor of the second PACCI measure, holding all things equally. The adjusted $\mathrm{R}^{2}$ value for this model is .105 .

\section{Table 21}

Estimation Results. DV: PACCI Case Studies; Additional IV: Full PACCS Scale

\begin{tabular}{|c|c|c|c|c|}
\hline Variables & PACC1 & $\begin{array}{l}\text { Std. } \\
\text { Beta }\end{array}$ & PACCI2 & $\begin{array}{l}\text { Std. } \\
\text { Beta }\end{array}$ \\
\hline Constant & $1.024 * * *(.253)$ & & $.909 * * *(.267)$ & \\
\hline PACCS & $0.000(.019)$ & & $0.014(.026)$ & \\
\hline PSM & $0.026(.017)$ & & $0.018(.023)$ & \\
\hline Exposure to Diversity & $0.021(.018)$ & & $0.013(.023)$ & \\
\hline CoBRAS & $-0.040 * *(.019)$ & -.193 & $0.067 * * *(.025)$ & -.234 \\
\hline Experience with Discrimination & $0.020 *(.012)$ & .137 & $0.026 *(.014)$ & .134 \\
\hline Social Desirability Bias Scale & $-0.001(.024)$ & & $0.014(.036)$ & \\
\hline Two or More Languages Spoken & $0.016(.039)$ & & $0.010(.047)$ & \\
\hline Lived or Studied Abroad & $0.009(.013)$ & & $0.016(.017)$ & \\
\hline Age & $0.026(.018)$ & & $0.014(.030)$ & \\
\hline Male & $0.007(.031)$ & & $-0.019(.042)$ & \\
\hline Black/African American & $-0.080 * *(.038)$ & -.155 & $-0.129 * *(.055)$ & -.182 \\
\hline Asian & $-0.024(.055)$ & & $0.037(.066)$ & \\
\hline Other Race & $-0.047(.047)$ & & $-0.040(.072)$ & \\
\hline Observations & 195 & & 195 & \\
\hline R Squared & .2038 & & .2250 & \\
\hline Adj. R Squared & .081 & & .105 & \\
\hline $\mathrm{F}$ & $2.12 * * *$ & & $2.33 * * *$ & \\
\hline
\end{tabular}

Note: School fixed-effects employed; robust standard error in parentheses; ${ }^{*} \mathrm{p}<0.10 ; * *$ $\mathrm{p}<0.05 ; * * * \mathrm{p}<0.01$ 


\section{Summary}

This chapter served to answer the second research question. The PACCS results supported hypotheses related to PSM, CoBRAS, and Lifetime Experience with Discrimination; whereas the PACCI results supported hypotheses related to CoBRAS, and Lifetime Experience with Discrimination. Neither instrument supported the hypothesis related to MPA Exposure to Diversity or for the hypothesis testing the relationship between the PACCS and PACCI. The next chapter of the dissertation

explores the implications of these results in the context of the MPA curriculum and the discipline. 


\section{CHAPTER 7}

\section{DISCUSSION}

The focus on cultural competence as a worthwhile area of study within public administration has intensified over the past decade. Unlike other disciplines, there is not an agreed upon definition for what cultural competence means in the public administration context; nor an agreed upon expectation of what cultural competencies public administrators should demonstrate. In addition to a lack of a discipline specific conceptual framework for cultural competence, there has been little to no empirical research related to the predictors of cultural competence for public administrators. The purpose of this dissertation was to fill these gaps within the literature by creating a discipline specific definition and related competencies, while conducting empirical research to explore the predictors of cultural competence for graduating MPA students.

The first contribution of this study is in the form of a public administration specific definition of cultural competence and a set of competencies. This finding answers research question one. The second contribution of this study is the PACCI and PACCS assessment tools. The study supports a two-factor solution for the PACCI and a trimmed three-factor solution for the PACCS. Furthermore, the study fully supports the hypotheses for Lifetime Experience with Discrimination and CoBRAS, and partially supports the hypothesis for PSM. The study did not provide support for MPA Exposure to Diversity or the relationship between the PACCS and PACCI. This was the first study in the field of public administration to empirically identify a specific definition of cultural competence and set of related competencies for public administrators, as well as examine the predictors of cultural competence for public administration students. 
This chapter will discuss the findings for each of the research questions in more detail and in the context of the literature. It then reviews the study's limitations. The chapter concludes with implications for public administration education and training programs, and future directions for research.

\section{Summary of the Findings}

This section of the chapter presents a summary of the findings, organized by research question. The findings for each research question are discussed, interpreted, and situated in relation to existing literature.

\section{Research Question 1}

The first research question is: How is cultural competence measured in public administration? To answer this research question, the first phase of the study sought to define cultural competence and identify related cultural competencies that public administrators should be able to demonstrate, as well as develop direct and indirect measures for public administrator cultural competence.

Public administration specific definition and competencies. A thorough content analysis of similar disciplines and their cultural competence frameworks resulted in a definition and a set of 60 competencies. Seventeen higher-order competencies were included in the first round of the Delphi, and after completion of the Delphi study, the definition was refined and experts identified 27 related competencies. The distilled competencies mapped onto the proposed conceptual framework in Chapter 3. The validated definition of cultural competence for public administration included the key elements of the conceptual framework developed in Chapter 3. The final competencies 
align with previously presented, but not validated, cultural competencies that public administrators should be able to demonstrate (Rice \& Mathews, 2012; Carrizales, 2010).

Development of the PACCS and PACCI. In addition to the Delphi study used to create a definition and identify relevant competencies, phase 1 included the validation of the PACCS and PACCI assessment instruments. The 27 distilled competencies were narrowed down to 15 competencies to comprise the PACCS indirect assessment instrument. The PACCI included two short-answer case studies. While the PACCS results in the hypothesized three-factor CFA model, several items were trimmed, leaving 8 items in the final scale. The CFA for the PACCI resulted in a two-factor solution, one for each case study, as hypothesized.

PACCS as a predictor of PACCI. The full PACCS score was not significant for either PACCI factor. The relationship between the full PACCS and PACCI instruments is interesting due to the significant relationship between the PACCS and social desirability bias, and the fact that direct and indirect assessments provide very different measures of student learning.

Social desirability response bias depends on how transparent the self-report measures are to the person taking the assessment (Constantine, 2000). This research confirms that social desirability bias is significantly related to the PACCS for graduating MPA students. For direct measures, however, social desirability bias is not significant. This is an important finding, as it demonstrates the why direct assessments should be considered for measuring cultural competence, when feasible.

These findings remain consistent with previous research on cultural competence indirect measurements and social desirability bias (Constantine \& Ladany, 2000; Reyes, 
Hadley, \& Davenport, 2013; Bernhard et al., 2015; Worthington, Mobley, \& Tan, 2000; Sodowsky et al., 1998). Per Wiggins (1959), "social desirability response style may be defined as a general tendency to endorse personality inventory items that are judged to be socially acceptable by people in general" (p. 419). In terms of its relationship with cultural competence, results are mixed. Constantine (2000), Sodowsky et al. (1994), and Ponterotto et al (1996) found either no correlation, or insignificant correlations between social desirability and indirect multicultural competence assessments. Whereas, Constantine \& Ladany (2000), Reyes, Hadley, and Davenport (2013), Bernhard et al. (2015), Worthington, Mobley, \& Tan (2000), and Sodowsky et al (1998) did find correlations between social desirability and indirect multicultural competence assessments.

These results provide insight for future research on both direct and indirect measures. It is important to understand how to decrease the social desirability bias with the PACCS instrument to see if this would provide a more valid instrument, as well as a more equivalent measure to the PACCI direct assessment. Furthermore, although the PACCI was not significantly related to social desirability bias, it is important to note that interrater reliability must be taken into account, in addition to the time intensive nature of such an instrument. Further, since the factor loadings were lower for the PACCI, in particular for case 1 , alternative scenarios more similar to case 2 should be considered. An important next step would be to further develop and test both indirect and direct assessment instruments to gauge cultural competence awareness, knowledge, and skills. While direct measures appear to be an ideal measure for cultural competence due to their ability to pull out nuances in behavior, alternative indirect measures can be explored for 
use where direct measurement is not possible. These include assessments that present scenarios to students and then ask the students to rank the scenario on a scale according to the trait being assessment. Similar scales have been developed in business ethics (Reidenbach \& Robin, 1990).

\section{Research Question 2}

Phase two of the study addressed the second research question: What are the predictors of cultural competence for graduating MPA students? The main independent variables for this study were: PSM, MPA Exposure to Diversity, CoBRAS, and Lifetime Experience with Discrimination. To answer this question, OLS regression with robust errors was employed. The following discussion is organized by independent variable.

PSM. As noted in Chapter 2, the study included PSM because of the similarity between attributes that drive entry into public service and cultural desire. PSM is a significant predictor for the PACCS; it was not a significant predictor of either of the PACCI factor scores.

One possible explanation for the relationship with the PACCS scale and not the PACCI is that PSM moderately correlates to social desirability bias in this study (.32). While scholars researching PSM note the possible influence of social desirability bias, it is not explored in depth (Liu \& Perry, 2014; Wright, 2007; Perry, 1996). This would indicate that students who rate themselves highly on the social desirability bias scale would also be more likely to rate themselves highly on indirect measures that contain socially desirable responses, such as both PACCS and PSM. For the PACCI, while the relationship with PSM was positive, it was not significant. Since direct assessment measures require students to apply awareness and knowledge and demonstrate that they 
can not only identify cultural conflicts but also solve them, it is possible that PSM is an important driving factor of cultural competence, although it may not be a sufficient indicator of whether or not a student can demonstrate these behaviors in a given scenario. In the field of nursing, where this concept of cultural desire originated, further research is necessary in terms of how to best assess this construct (Camphina-Bacote, 2008). Thus, further research on this topic is warranted in several disciplines.

Methodologically, future studies should include private sector employees, as the influence of PSM may be more pronounced when comparing those from the public vs. private sector (Houston, 2000). In addition, extending the sample to account for additional variables such as highest level of education and length of employment may help to explain variance in levels of cultural competence. Overall, these findings highlight the need for an expanded theory behind the motivation of public administrators and how this motivation translates into demonstrating culturally competent behaviors.

MPA Exposure to Diversity. The second predictor of interest, MPA Exposure to Diversity, was not significant in any of the models. The construction of this variable is a limitation of the study. While the variable was not significant for either the PACCS or PACCI, it is important to note that the descriptive statistics indicate a relatively low perception of coverage. When looking at the individual variables that make up this scale, most students report that they have taken two or fewer courses that focused on diversity or cultural competence issues; feel that their required courses only emphasize cultural content "very little" to "somewhat"; and have attended less than one workshop or training related to these issues. 
This finding is inconsistent with the literature related to cultural competence training (Benkert et al, 2011; Price, 2005; Kardong-Edgren et al., 2005; Sodowsky et al., 1998; Crandall et al., 2003; Schim, Doorenbos, \& Borse, 2005; Schim, Doorenbos, \& Borse, 2006), higher education research related to diversity education within the classroom (Pike \& Kuh, 2006; Terenzini, et al., 2001), and cultural competence curriculum and student learning outcomes (Caffrey et al., 2005; Sargent, Sedlak \& Martsolf, 2005).

While inconsistent, it is important to note that the direct assessment scores demonstrate the need for additional exposure to cultural competence and problem-solving in this arena; increased classroom coverage, using the teaching strategies mentioned in this dissertation, can improve these outcomes. Therefore, future studies should include deeper analyses into the curriculum and mission statement of programs to better understand the level of exposure students are truly receiving, instead of relying on selfreported measures. Development of a curriculum assessment instrument, similar to the Association of American Medical Colleges' Tools for Assessing Cultural Competence Training (TACCT), can assist in this regard.

CoBRAS. The third predictor of interest, CoBRAS, was a significant predictor for the full PACCS score and both PACCI factor scores. Out of the predictor variables discussed thus far, CoBRAS is the first to be significant for both the indirect and direct measure of cultural competence. These findings are in line with previous research conducted on the relationship between CoBRAS and Multicultural Competence (Chao, 2006; Neville, Spanierman, \& Doan, 2006). 
While most of the research on CoBRAS is within the field of psychology, it offers important insight regarding a student's personal attitudes and beliefs, which can then impact their ability to serve the public in a culturally competent manner. Borrego \& Johnson (2012) list similar theoretical reasons for why obstacles to cultural competence occur related to CoBRAS, such as their contribution regarding white entitlement. This concept refers to "the notion of a self-made person who makes good despite all odds...[and] some people who cling to this belief do not want to discuss this issue of race and its effects on society" (pp. 15-16). This finding of the significance of CoBRAS is critical, as it demonstrates a tangible way to gauge core beliefs that may hinder cultural competence development, thereby opening opportunities to start a dialogue about how to mitigate these beliefs when serving the public.

Lifetime Experience with Discrimination. The next predictor of interest, Lifetime Experience with Discrimination, was significant for both the PACCS and PACCI. This finding indicates that students who have had more experiences with discrimination are more likely to score higher on both direct and indirect assessments of cultural competence. In terms of the types of experiences with discrimination that students reported, sexism (51.7\%) was the most common; followed by racism (47.2\%), ageism (30\%), classism (25.5\%), religious discrimination (24.3\%), homophobia (8.2\%), and ableism (4.1\%). This finding confirms previous research related to the topic (Mattis et al., 2004; White, 2008); however, it also fills a gap in the literature within public administration, demonstrating that these experiences impact a student's level of cultural competence. 
This finding is important, as it demonstrates that people who experience acts of discrimination are more likely to demonstrate culturally competent awareness, knowledge, and behaviors. In relation to MPA program curriculum, it becomes necessary to understand how to expose students who do not experience discrimination to why these acts impact one's day to day behavior. While there is no substitute for personally experiencing what it is like to be a member of a marginalized group in society, encouraging dialogue on the topic may serve to highlight those experiences and why it is important to not only recognize but address biases and stereotypes.

\section{Implications for Public Administration MPA Curriculum}

As noted in the final chapter of the comprehensive Cultural Competence for

\section{Public Administrators:}

In order for public administrators to be able to assess the budgetary, legal, programmatic, and evaluative aspects of cultural competency in public services, considerations of cultural competence must become second nature to their jobs. This suggests it must be interwoven into all aspects of their education and training as public servants. Getting to this point requires that public affairs programs at all levels incorporate cultural competency skills and knowledge into courses across the curriculum and not leave this topic to select courses or electives. While several programs appear to be working toward this end, there is still much to do (pp. 350-351).

The purpose of this section of the dissertation is to provide suggestions for how programs can accomplish this, based on the findings from this study and best practices referenced in the literature. Borrego and Johnson (2012) and Gooden and Norman-Major (2012) note several key challenges for integrating cultural competence into the MPA program: faculty preparation, course content and delivery, and assessment. The findings of this dissertation provide guidance on each of these factors. In terms of faculty preparation, the program director survey for this dissertation indicated that while there is 
interest in increasing coverage of cultural competence across their curriculum (93.75\%), only $62.50 \%$ indicated that they have adequate faculty specialization to achieve this coverage. This indicates that graduating students from not only MPA programs, but $\mathrm{PhD}$ programs as well, are lacking exposure to cultural competence; and in the case of $\mathrm{PhD}$ students, will not be able to adequately teach courses related to this material. The suggestions made in this dissertation help to address this problem by providing programs with assessable competencies, as well as providing insight into theories that may help students become motivated towards cultural competence.

In terms of course content and delivery, $68.75 \%$ of the programs included in this study had at least one core course with a focus on cultural competence or diversity, defined as having at least one student learning outcome related to these areas. For elective courses, $50 \%$ of the programs had at least two electives with such a focus, $12.5 \%$ noted 3 courses, and $31.25 \%$ noted more than three courses. In terms of referencing cultural competence in all selected courses, $68.75 \%$ said that these topics are "somewhat" emphasized. This data provides evidence that cultural competence coverage occurs in select courses and is usually not infused throughout the program of study. The competencies provided by this study provide guidance as to what should be assessed; an extension of this study can map the validated competencies to core MPA courses.

The above challenges tie into the assessment requirements required by the NASPAA universal competency related to cultural competence. According to Rubaii and Calarusse (2012), "defining what constitutes cultural competency is a challenge, but it is eclipsed by the difficulty of measuring the competency" (p. 239). The findings from this dissertation addresses the definitional aspect, and provide evidence regarding how case 
studies can gauge a student's level of cultural competence. The program directors survey indicates that when asked to rank the universal competencies in order from easiest to hardest to assess, $47.67 \%$ indicated that "to communicate and interact productively with a diverse and changing workforce and citizenry" was the most difficult. Furthermore, $56.25 \%$ of the sample indicated that they do not have enough NASPAA support regarding assessment efforts for all the universal competencies. When asked how they assess cultural competence, answers ranged from not assessing the competence directly:

We do not assess this explicitly. We do assess diversity issues as part of competency 4 , for which students must submit a portfolio, but they could satisfy that without discussing cultural competency.

Not assessing the competency at all:

We really don't. We ask questions around the topic, but nothing that I would consider cultural competency.

To greater integration throughout the curriculum:

Cultural competency is associated with learning outcomes that fall under several of the NASPAA competency domains. For example, we have defined expected student learning outcomes for cultural competency that fall under communication (to diverse audiences, multiple media), problems solving and decision making, and policy process (identifying and including diverse stakeholders, representativeness). Assessment for our annual assessment of learning comes through our required comp exams, administered after all required core classes have been completed. The exam includes questions that incorporate cultural competency components--it is not a standalone exam/question.

The range of these responses indicates that assessment of cultural competence needs to be addressed throughout many MPA programs. Program directors noted that a cultural competence toolkit, additional assessment training from NASPAA, additional texts devoted to the subject, and examples of approaches for how to assess related 
competencies would all be helpful to expand cultural competence throughout the curriculum.

Norman-Major (2012) provides a comprehensive framework for where cultural competence can be assessed within a program, as well as an example of assessment instruments; this dissertation supplements this framework with specific competencies. Furthermore, it also provides insight into the theoretical foundation behind cultural competence in public administration, each of which can play a role in how faculty approach cultural competence in their courses.

For example, the literature demonstrates that a dialogic model as well as teambased learning are both effective teaching tools for this topic (Rivera, Johnson, \& Kodaseet, 2012; Saldivar, 2015). While professors will have specific learning outcomes for each lesson when using these tools, the exploration of CoBRAS or experiences with discrimination can create a dialogue, which may result in attitudinal change or increased cultural awareness. In terms of team-based learning, creating teams where students can share diverse experiences related to discrimination would also be an effective means to increase awareness.

In addition, this dissertation argues that an increased focus is needed on democratic ethos as opposed to bureaucratic ethos to develop skills that relate to PSM and may therefore increase a student's willingness to engage in culturally competent behaviors. An enhanced coverage of virtue ethics can serve this purpose. When approaching a public service delivery issue from the scope of principle-based deontological and teleological ethics, the automation of decisions may occur, leading to decisions that may meet the "bottom line," but negatively impact social welfare 
(Marchese, Bassham, \& Ryan, 2002, p. 152). Virtue ethics, however, determines whether an act is "good" based upon the character trait or virtue that the act evidences, which can ultimately lead to decisions that are more beneficial (Geuras \& Garafolo, 2010, p. 59). Cultural competence fits well within the umbrella of virtue ethics, as it requires a contextual analysis to make an appropriate decision. This type of coverage can help students gain exposure to virtues that may impact their level of PSM, which can lead to increased levels of cultural competence.

While Norman-Major's framework is excellent, a more explicit framework which includes student learning outcomes tied to the competencies outlined in this study may provide even better guidance for program directors and faculty members alike. While a cultural competence specific framework is useful, programs are challenged to create a competency cross-walk for the entire program. This challenges programs to explore all of the competencies that they wish to cover and better understand how these competencies can work together in different courses. While all 27 competencies developed as part of the Delphi study are important; programs may want to focus on the 8 final competencies presented as part of the PACCS to implement across the curriculum. The remaining competencies may be reserved for elective courses that focus specifically on cultural competence.

The overall implications for MPA curriculum are three-fold. First, in accordance with Cross' model (1989), MPA programs must value cultural competence as critical, and demonstrate this commitment through relevant content in all courses. To produce culturally competent MPA graduates, it is imperative to help students move along the cultural continuum -from cultural blindness to cultural proficiency- programs can 
accomplish this by integrating concepts into each one of the MPA core courses. Cross' step of Developing Cultural Competence can be carried out at the accreditation and program level. NASPAA can help with this through providing additional assessment guidance to programs, as well as holding programs accountable by requiring detailed assessment plans that demonstrate curricular-wide integration. MPA program directors and faculty can use the definition and competencies developed by this dissertation as a starting point to understand what their students should be able to demonstrate by the time that they graduate.

Second, the findings from this dissertation demonstrate that social desirability bias is a significant influence on indirect measures of cultural competence, and should not be depended upon for an accurate assessment of these skills. Until the PACCS is further tested to remove this bias, programs are encouraged to use direct measures to examine student learning related to cultural competence.

Third, faculty preparation is key to ensuring cultural competence coverage. This aligns with Cross' last step of Planning for Cultural Competence. To adequately plan, programs must have support and build resources to adequately prepare faculty. Since this seems to be a difficult area for many programs, hiring committees should value applicants with this skill set and recruit faculty specifically for this purpose. While these faculty members could teach a course strictly related to diversity or cultural competence, they are also an invaluable resource for integrating these skills within other core courses.

\section{Implications for Public Administration Training Programs}

In addition to the recommendations related to MPA programs, some of the results are also generalizable to public organizations. This study provides several important 
findings that public organizations can utilize to further the cultural competence growth of their employees and practices. Per Cross' 1989 Model for Cultural Competence, organizations must first understand that cultural competence development happens on the continuum, and then commit to implementing practices that promote responsiveness. This research helps to guide the third step of Cross' model, which is "Developing Cultural Competence." Through the competencies developed in this study, managers and other high level organization officials have access to a list of the skills that they and their employees should be able to demonstrate. In addition to this list of competencies, other resources, such as Rice's checklist (2007b), and Borrego and Johnson's (2012) measures for cultural competence included throughout their book provide additional guidance for how to ensure that their organization promotes cultural competence and continually develops along the continuum. The findings from this research can also help to facilitate Cross' final step "Planning for Cultural Competence", as the definition and related competencies can play a role in the strategic planning process for an organization.

Overall, organizations must commit to cultural competence and examine how their missions and practices meet the definition provided in this study. It is important to understand the theoretical drivers of cultural competence presented here as well. If an issue arises with an employee, it is helpful to understand how to increase his/her competence, by understanding the individual's implicit attitudes.

\section{Limitations}

The first limitation is the reliance on self-reported measures of cultural competence exposure within the MPA program. Future studies should examine a more robust way to address collecting data for this variable. An additional limitation relates to 
the inability to run a higher order structural equation model with the PACCS and PACCI. While individually each scale provides significant results, using a higher order factor would provide more information on using these instruments together. The third limitation is the low composite reliability and validity of the PACCI1 factor; however, due to the well-fitting overall model for both PACCI factors, and congruent regression results for both, this limitation is adequately addressed within the study. Future research may benefit from focusing on higher-order direct measures, such as the second factor.

Furthermore, it is important to discuss two potential methodological limitations: the cross-sectional nature of the study and common source bias. While cross-sectional studies provide several advantages, reverse-causality is a distinct limitation (Wright \& Grant, 2010). In this study, reverse causality must be considered for PSM and CoBRAS. The hypothesized relationship between PSM and cultural competence is supported based on an understanding that underlying characteristics such as compassion, commitment to social justice, and caring are developed over time, beginning in childhood (Perry, 1997; Camphina-Bacote, 2008). Furthermore, cultural competence requires the development of awareness, knowledge, and skills that must be acquired through specific interactions. As noted in the literature, the need for expansive training programs and curricular integration demonstrates that these skills must be developed conscientiously (Rice, 2007b). Thus, higher levels of PSM, as with cultural desire, would indicate that a person has a specific disposition that makes them more likely to seek out education to develop the skills required to be culturally competent.

To support the hypothesized relationship between CoBRAS and cultural competence, a similar argument is presented. Colorblind racial attitudes, much like PSM, 
are formed over time as a result of "the broader structure of race relations in the United States" (p. 270). According to Neville, Worthington, \& Spanierman (2001), multiculturally competent counselors must use the awareness, knowledge, and skills that they have developed to critically evaluate how their colorblind racial attitudes impact their behavior. The authors further argue that "the assumption is that not everyone will be ready to accurately encode, interpret and integrate the new race-related content at initial critical exposure" (p. 278), indicating that colorblind racial attitudes exist prior to an individual developing the cultural competence to understand how these attitudes impact their behavior.

The second methodological limitation to the study is common source bias (CSB). CSB "indicates potential issues when scholars use the same data source, typically a survey, to measure both independent and dependent variables simultaneously" (George \& Pandey, 2017). George and Pandey (2017) present four questions in evaluating a study in light of CSB. The first is whether or not the "common method variance is high enough to generate CSB", which can be found using Harman's one-factor test. The results of this test for the data used in the dissertation indicate that a one-factor solution accounts for $21.5 \%$ of the variance in the data, much lower than the $50 \%$ cutoff proposed in the literature (Fuller et al., 2016). The second is whether the variables in the study are among those that have been found to produce CSB, including organizational performance, individual performance, and self-reported data. While the study does include self-report measures, it included specific instructions to encourage honest responses, controlled for social desirability, and included the PACCI, which as a direct measure of cultural competence, provides an independent variable that does not rely on recall or self-reported 
measures. These factors are procedural remedies which help to mitigate any potential CSB (Podsakoff, MacKenzie, \& Podsakoff, 2012). The third question posed by George and Pandey is whether or not the survey responses are perceptual and can only be measured through a survey, which is the case for this dissertation. The last question posed is: "are other data sources nonexistent, irrelevant, or of poor quality, and if a survey is used, are multiple items used to measure variables and is the scale reliability (i.e., Cronbach's $\alpha$ ) acceptable (Fuller et al., 2016)?” (George \& Pandey, 2017). For this dissertation, external or archival data were not available or appropriate; and where possible, multiple items were used to measure variables that results in acceptable Cronbach's alpha levels. Based on the Harman's one factor test, and the procedural remedies taken into consideration, CSB is not found to be of concern in the study.

A final limitation of the study is that the results are only generalizable to graduating MPA students. The definition and competencies developed, however, can be applied in both educational and professional contexts.

\section{Directions for Future Research}

The results of this dissertation point to three important avenues for future research: direct assessment in MPA programs, direct assessment in public sector organizations, and cultural climate studies. While the PACCS demonstrated a significant relationship with social desirability bias, and did not have a significant relationship with the PACCI, future research should focus on further validating the PACCS, in both MPA and workforce contexts. The measurement model results in a significantly trimmed scale, thus it would be of interest to see if larger samples, and samples including public administration professionals, result in the same findings. 
As this was the first study of its kind to examine direct measures of cultural competence, further validation and testing is required. Due to the inability to measure both instruments on one higher order factor score, it was not possible to run a full structural equation model with both the indirect and direct instruments. A study that expands upon this concept may be able to better make the argument for a combined approach to cultural competence assessment. Overall, more attention should be placed on developing a direct measure that provides valid and reliable results in both educational and professional settings, to reduce the need for an indirect measure.

Due to the lack of empirical research in the field on cultural competence measurement, it is critical to examine additional theoretical foundations that can help explain a public administrator's level of cultural competence. While each model exhibited acceptable $\mathrm{R}^{2}$ levels, there may be additional underlying factors that can be leveraged to improve cultural competence skills within both graduating and seasoned public administrators.

The results of the regression analyses also provided several areas where further research is warranted. In addition to PSM, researchers should examine other types of motivation or attitudinal attributes to determine relevant drivers of cultural competence. In terms of MPA Exposure to Diversity, future research should examine this on a more granular basis, through evaluation of syllabi, transcripts, and extra-curricular programming. Including the CoBRAS scale in future studies will help to further cement this part of the theoretical foundation of cultural competence; and additional research related to Lifetime Experience with Discrimination will be useful for this same purpose. 
A third avenue for future research is expanding research on measuring cultural competence at the individual level to examine cultural climate at the program and organizational level. This avenue of research is important, as the culture of an organization can heavily influence employees and their behavior. The literature related to cultural climate, in the context of cultural competence, is limited to suggestions for selfstudy and self-assessment (Rice, 2007; Matthews, 2010). It is important to go beyond self-assessment into formal evaluations, by putting forth and testing theories that can generate meaningful interventions and implications for organizations.

This dissertation succeeded in providing evidence for interventions within MPA programs to improve cultural competence. In addition, it sets the stage for future research with public sector employees. In the long term, future research should focus on organizational cultural climate studies, and how cultural competence can be leveraged to address climate issues. 


\section{CHAPTER 8}

\section{REFLECTION}

This dissertation makes an important contribution to the study of cultural competence in public administration for three main reasons. First, it ties together research from various public administration scholars to create a cohesive definition for cultural competence. In addition, it validated a set of competencies required of public administrators. Second, it contributes to a theoretical base for cultural competence in public administration, demonstrating the importance of PSM, CoBRAS, and Lifetime experience with discrimination in shaping cultural competence for future public administrators. Lastly, it sets the stage for additional empirical research, including a focus on validating the PACCS with public sector employees, and broadening the focus of cultural competence to also include examining cultural climate within public organizations.

The pillar of social equity has received the least attention in comparison to efficiency, economy, and effectiveness. However, given the current political climate, it is now more apparent than ever that equity, diversity, and cultural competence are critical. As the nation continues to diversify, it is necessary to understand how culture has shaped political power and what that means for the future of the United States. This dissertation provides a window into how culturally competent future public administrators are, and demonstrates that MPA programs and public organization training programs must pay attention to how their students and employees are developing these necessary competencies to better serve an increasingly diverse citizenry. 
While this dissertation serves theoretical and practical purposes, a primary goal of this research was to assess the need to take research related to social equity and cultural competence seriously. The empirical evidence demonstrates that it is possible to employ regression analyses to predict cultural competence, and that empirical research on the topic can be interesting and valuable. Furthermore, the student case study answers are indicators that our future public administrators may not be receiving the education they need to address complex issues related to culture. Cultural competence must be viewed as an issue of moral imperative in our current climate. As evidenced by this dissertation, public administration scholars and practitioners can begin to take steps to incorporate issues of equity into their research as well as infuse equity and cultural competence across the MPA curriculum.

Overall, the most important finding of this study is that many students believe they possess cultural competence when they are unable to demonstrate it. This presents an issue for programs who tout that they graduate students prepared to serve a culturally diverse citizenry. Given the current climate in the United States, training public servants to be mindful of different cultures is critical, and MPA programs must better integrate the competencies presented in this dissertation across their curriculum. Infusing cultural competence does not mean offering stand-alone courses or certificates; it means exposing students to how issues of diversity, equity, and cultural issues span all areas, from human resources to budgeting. Commitment to such an infusion can serve as a driving force to elevate the "human" side of public administration; bringing to light the importance of related topics such as emotional intelligence, virtue ethics, and social equity. Without this 
commitment, MPA programs are not only graduating ill-prepared public servants, but also failing the public.

\section{Conclusion}

While cultural competence has continued to gain credibility within the field of public administration, there was a significant gap in the literature related to how to define this concept and agreement about what cultural competencies are required of public administrators. Furthermore, there was a significant gap related to the theoretical foundation of cultural competence in public administration, in addition to an absence of empirical research on the topic.

This study filled these gaps through employing a two-phase mixed-method research design. In terms of implications for practice, the results of the study present the definition and set of competencies. The study also contributes to theory, as the results demonstrate a relationship between indirect measures of cultural competence with PSM, CoBRAS, and Lifetime Experience with Discrimination; and a relationship between direct measures of cultural competence with CoBRAS and Lifetime Experience with Discrimination. While future research is needed to further confirm these findings, the results demonstrate that indirect measures of cultural competence assessment are influenced by social desirability bias, while direct measures are not; indicating the importance of further investigating the use of direct measures on this topic. The findings of this study confirm the need for culturally competent public administrators due to the rising focus on issues of inequity and social justice, and provide both practical guidance 
for MPA programs and public administration training programs, and theoretical guidance for future empirical research. 


\section{REFERENCES}

Allison, K. W., Echemendia, R. J., Crawford, I., \& Robinson, W. L. (1996). Predicting cultural competence: Implications for practice and training. Professional Psychology: Research and Practice, 27(4), 386.

Alonso, P., \& G. Lewis. (2001). Public service motivation and job performance: Evidence from the federal sector. American Review of Public Administration 31, 363-80.

American Association of Colleges of Nursing. (2009). Establishing a culturally competent master's and doctorally prepared nursing workforce. Retrieved from http://www.aacn.nche.edu/education-resources/CulturalComp.pdf

American Psychological Association. (2013). Guidelines and principles for accreditation of programs in professional psychology. Washington, D.C.

Ang, S., Van Dyne, L., \& Koh, C. (2006). Personality correlates of the four-factor model of cultural intelligence. Group \& Organization Management, 31(1), 100-123.

Association of American Medical Colleges, (2005). Cultural competence education. Washington, D.C.

Awad, G. H., Cokley, K., \& Ravitch, J. (2005). Attitudes toward affirmative action: A comparison of color-blind versus modern racist attitudes. Journal of Applied Social Psychology, 35(7), 1384-1399.

Bailey, M. L. (2005). Cultural competency and the practice of public administration. In M. F. Rice (Ed.), Diversity and public administration: Theory, issues, and perspectives (pp. 177-196). Armonk, NY: M. E. Sharpe.

Barrett, P. (2007). Structural equation modelling: Adjudging model fit. Personality and Individual Differences, 42(5), 815-824.

Benkert, R., Templin, T., Schim, S. M., Doorenbos, A. Z., \& Bell, S. E. (2011). Testing a multi-group model of culturally competent behaviors among underrepresented nurse practitioners. Research in Nursing \& Health, 34(4), 327-341.

Bernhard, G., Knibbe, R. A., von Wolff, A., Dingoyan, D., Schulz, H., \& Mösko, M. (2015). Development and psychometric evaluation of an instrument to assess cross-cultural competence of healthcare professionals (CCCHP). PloS One, 10(12), e0144049.

Berry-James, R. M. (2012). Cultural competency in health care: Standards, practices and measures. In K. Norman-Major \& S. Gooden (Eds.), Cultural competency for public administration (pp. 181-196). Armonk, NY: M.E. Sharpe. 
Bonilla, J.F., Lindeman, L., \& Taylor, N. (2012). Educating for and assessing cultural competence. In K. Norman-Major \& S. Gooden (Eds.), Cultural competency for public administration (pp. 294-309). Armonk, NY: M.E. Sharpe.

Borrego, E. \& Johnson, R.G. (2012). Cultural competence for public managers: Managing diversity in today's world. Boca Raton, FL: CRC Press.

Bresciani, M. J., Oakleaf, M., Kolkhorst, F., Nebeker, C., Barlow, J., Duncan, K., \& Hickmott, J. (2009). Examining design and inter-rater reliability of a rubric measuring research quality across multiple disciplines. Practical Assessment, Research \& Evaluation, 14(12), 1-7.

Caffrey, R.A., Neander, W., Markle, D., \& Stewart, B. (2005). Improving the cultural competence of nursing students: Results of integrating cultural content in the curriculum and an international immersion experience. Journal of Nursing Education, 44, 234-240.

Campinha-Bacote, J. (2002). The process of cultural competence in the delivery of healthcare services: A model of care. Journal of Transcultural Nursing, 13(3), 181-184.

Campinha-Bacote, J. (2008). Cultural desire: 'Caught' or 'taught'? Contemporary Nurse, 28(1-2), 141-148.

Carrizales, T. (2010). Exploring cultural competency within the public affairs curriculum. Journal of Public Affairs Education, 16(4), 593-606.

Chae, D. H., Park, Y. H., Kang, K. H., \& Lee, T. H. (2012). A study on factors affecting cultural competency of general hospital nurses. Journal of Korean Academy of Nursing Administration, 18(1), 76-86.

Chao, R. (2006). Counselors' multicultural competencies: Race, training, ethnic identity, and color-blind racial attitudes. Vistas: Compelling Perspectives on Counseling, Article 15, 73-76.

Chao, R. C. L., Wei, M., Good, G. E., \& Flores, L. Y. (2011). Race/ethnicity, color-blind racial attitudes, and multicultural counseling competence: the moderating effects of multicultural counseling training. Journal of Counseling Psychology, 58(1), 7282.

Christensen, R. K., \& Wright, B. E. (2011). The effects of public service motivation on job choice decisions: Disentangling the contributions of person-organization fit and person-job fit. Journal of Public Administration Research and Theory, 21(4), 723-743. 
Constantine, M. G., \& Ladany, N. (2000). Self-report multicultural counseling competence scales: Their relation to social desirability attitudes and multicultural case conceptualization ability. Journal of Counseling Psychology, 47(2), 155-164.

Council on Education for Public Health. (2013). Accreditation criteria: Standalone baccalaureate programs. Silver Spring, MD.

Cram, B. \& Alkadry, M. (2018). Social Equity Leadership. In A. Farazmand (Ed.) Global encyclopedia of public administration and public policy. Springer.

Crandall, S. J., George, G., Marion, G. S., \& Davis, S. (2003). Applying theory to the design of cultural competency training for medical students: a case study. Academic Medicine, 78(6), 588-594.

Cross T., Bazron, B., Dennis, K., \& Isaacs, M. (1989). Towards a culturally competent system of care, volume I. Washington, D.C.: Georgetown University Child Development Center, CASSP Technical Assistance Center

Curran, P. J., West, S. G., \& Finch, J. F. (1996). The robustness of test statistics to nonnormality and specification error in confirmatory factor analysis. Psychological Methods, 1(1), 16-29.

Denboba, D., (1993). MCHB/DSCSHCN guidance for competitive applications, maternal and child health improvement projects for children with special health care needs. U.S. Department of Health and Human Services, Health Services and Resources Administration.

Denhardt, K. G. (1997). The management of ideals: A political perspective on ethics. International Journal of Public Administration, 20(4-5), 1091-1115.

Denhardt, R. B., \& Denhardt, J. V. (2000). The new public service: Serving rather than steering. Public Administration Review, 60(6), 549-559.

Ekblad, S., Marttila, A., \& Emilsson, M. (2000). Cultural challenges in end-of-life care: reflections from focus groups' interviews with hospice staff in Stockholm. Journal of Advanced Nursing, 31(3), 623-630.

Elías, M. V., \& Alkadry, M. G. (2011). Constructive conflict, participation, and shared governance. Administration \& Society, 43, 869-895.

Frederickson, G. (2005). The state of social equity in American public administration. National Civic Review, 94(4), 31-38.

Frederickson, H. G. (2010). Social equity and public administration; origins, developments, and applications. London, England: M.E. Sharpe. 
Fuller, C. M., Simmering, M. J., Atinc, G., Atinc, Y., \& Babin, B. J. (2016). Common methods variance detection in business research. Journal of Business Research, 69(8), 3192-3198.

George, B., \& Pandey, S. K. (2017). We know the yin—but where is the yang? Toward a balanced approach on common source bias in public administration scholarship. Review of Public Personnel Administration. Advance online publication

Gierl, M. J., \& Mulvenon, S. (1995). Evaluating the application of fit indices to structural equation models in educational research: A review of the literature from 1990 through 1994. In annual meeting of the American Educational Research Association, San Francisco, CA.

Gooden, S., \& Portillo, S. (2011). Advancing social equity in the Minnowbrook tradition. Journal of Public Administration Research and Theory, 21(suppl 1), i61-i76

Goss, R. P. (1996). A distinct public administration ethics? Journal of Public Administration Research and Theory, 6(4), 573-597.

Gurin, P., Dey, E., Hurtado, S., \& Gurin, G. (2002). Diversity and higher education: Theory and impact on educational outcomes. Harvard Educational Review, 72(3), 330-367.

Hair, J., Anderson, R., Tatham, R., \& Black, W. (1995). Multivariate data analysis. New Jersey: Prentice-Hall Inc.

Hair, J., Black, W., Babin, B., \& Anderson, R. (2010). Multivariate data analysis. Upper Saddle River, NJ. Prentice-Hall, Inc.

Hallgren, K. A. (2012). Computing inter-rater reliability for observational data: an overview and tutorial. Tutorials in Quantitative Methods for Psychology, 8(1), 2334.

Hansson, E. E., Svensson, P. J., Strandberg, E. L., Troein, M., \& Beckman, A. (2014). Inter-rater Reliability and Agreement of Rubrics for Assessment of Scientific Writing. Education, 4(1), 12-17.

Hasson, F., Keeney, S., \& McKenna, H. (2000). Research guidelines for the Delphi survey technique. Journal of Advanced Nursing, 32(4), 1008-1015.

Hooper, D., Coughlan, J., \& Mullen, M. (2008). Structural equation modelling: Guidelines for determining model fit. Electronic Journal of Business Research Methods, 6(1), 53-60.

Houston, D. J. (2000). Public-service motivation: A multivariate test. Journal of Public Administration Research and Theory, 10(4), 13-727. 
Houston, D. J. (2006). "Walking the walk" of public service motivation: Public employees and charitable gifts of time, blood, and money. Journal of Public Administration Research and Theory, 16(1), 67-86.

Hsu, C. C., \& Sandford, B. A. (2007). The Delphi technique: making sense of consensus. Practical Assessment, Research \& Evaluation, 12(10), 1-8.

Hu, L. T., \& Bentler, P. M. (1999). Cutoff criteria for fit indexes in covariance structure analysis: Conventional criteria versus new alternatives. Structural Equation Modeling: A Multidisciplinary Journal, 6(1), 1-55.

Hutcheson, G. D. (2011). Ordinary Least-Squares Regression (pp. 224-228). In L. Moutinho and G. D. Hutcheson, The SAGE Dictionary of Quantitative Management Research. Thousand Oaks, CA: Sage Publishing.

Johnson, R. B., \& Onwuegbuzie, A. J. (2004). Mixed methods research: A research paradigm whose time has come. Educational Researcher, 33(7), 14-26.

Kardong-Edgren, S., Bond, M. L., Schlosser, S., Cason, C., Jones, M. E., Warr, R., \& Strunk, P. (2005). Cultural attitudes, knowledge, and skills of nursing faculty toward patients from four diverse cultures. Journal of Professional Nursing, 21(3), 175-182.

Kaufman, H. (1956). Emerging conflicts in the doctrines of public administration. American Political Science Review, 50(04), 1057-1073.

Kenny, D. A. (2015, November 24). SEM: Fit. Retrieved January 28, 2017, from http://davidakenny.net/cm/fit.htm

Kitsantas, A. (2004). Studying abroad: The role of college students' goals on the development of cross-cultural skills and global understanding. College Student Journal, 38(3). 441-452.

Kohli, H. K., Kohli, A. S., Huber, R., \& Faul, A. C. (2010). Assessing Cultural Competence in Graduating Students. International Journal of Progressive Education, 6(1), 6-27.

Koskinen, L., \& Tossavainen, K. (2004). Study abroad as a process of learning intercultural competence in nursing. International Journal of Nursing Practice, 10(3), 111-120.

Kumas-Tan, Z., Beagan, B., Loppie, C., MacLeod, A., \& Frank, B. (2007). Measures of cultural competence: examining hidden assumptions. Academic Medicine, 82(6), 548-557. 
Laird, T. F. N. (2005). College students' experiences with diversity and their effects on academic self-confidence, social agency, and disposition toward critical thinking. Research in Higher Education, 46(4), 365-387.

Lewis, H., Lewis, A., \& Williams, F. (2012). Cultural competency in public administration programs. In K. Norman-Major \& S. Gooden (Eds.), Cultural competency for public administration (pp. 244-264). Armonk, NY: M.E. Sharpe.

Lim Choi, D. (2004). Public service motivation and ethical conduct. International Review of Public Administration, 8(2), 99-106.

Linstone, H.A. and Turoff, M. (Eds.) (2002), The delphi method: Techniques and applications. Addison-Wesley Publishing Company Inc.

Liu, B., \& Perry, J. L. (2016). The psychological mechanisms of public service motivation: A two-wave examination. Review of Public Personnel Administration, 36(1), 4-30.

Longoria, T., \& Rangarajan, N. (2015). Measuring public manager cultural competence: The influence of public service values. Journal of Public Management \& Social Policy, 21(1), 24-41.

Lopez-Littleton, V., \& Blessett, B. (2015). A framework for integrating cultural competency into the curriculum of public administration programs. Journal of Public Affairs Education, 21(4), 557-574.

Loughlin K.G. and Moore L.F. (1979). Using delphi to achieve congruent objectives and activities in a paediatrics department. Journal of Medical Education, 54(2), 101106.

Lumley, T., Diehr, P., Emerson, S., \& Chen, L. (2002). The importance of the normality assumption in large public health data sets. Annual Review of Public Health, 23(1), 151-169.

Mastracci, S. H., Newman, M. A., \& Guy, M. E. (2010). Emotional labor: Why and how to teach it. Journal of Public Affairs Education, 16(2), 123-141.

Matsunaga, M. (2015). How to factor-analyze your data right: do's, don'ts, and how-to's. International Journal of Psychological Research, 3(1), 97-110.

Mattis, J. S., Beckham, W. P., Saunders, B. A., Williams, J. E., Myers, V., Knight, D., ... \& Dixon, C. (2004). Who will volunteer? Religiosity, everyday racism, and social participation among African American men. Journal of Adult Development, 11(4), 261-272. 
McConahay, J. B. (1983). Modern racism and modern discrimination: The effects of race, racial attitudes, and context on simulated hiring decisions. Personality and Social Psychology Bulletin, 9(4), 551-558.

NASPAA Data Center (2014). Retrieved from http://www.naspaa.org/DataCenter/ index.asp

National Academy of Public Administration. 2010. Standing panel on social equity in governance. Retrieved from http://www.napawash.org/fellows/standingpanels/standing-panel-on-social-equity-in-governance/

Neville, H. A., Lilly, R. L., Duran, G., Lee, R. M., \& Browne, L. (2000). Construction and initial validation of the color-blind racial attitudes scale (CoBRAS). Journal of Counseling Psychology, 47(1), 59-70.

Neville, H., Spanierman, L., \& Doan, B. T. (2006). Exploring the association between color-blind racial ideology and multicultural counseling competencies. Cultural Diversity and Ethnic Minority Psychology, 12(2), 275-290.

Neville, H. A., Worthington, R. L., \& Spanierman, L. B. (2001). Race, power, and multicultural counseling psychology: Understanding white privilege and colorblind racial attitudes. J.G. Ponterotto, J. Casas, L. Suzuki, C. Alexander (Eds). Handbook of multicultural counseling, 2nd ed. (pp. 257-288). Thousand Oaks, CA, US: Sage Publications, Inc.

Newman, M. A., Guy, M. E., \& Mastracci, S. H. (2009). Beyond cognition: Affective leadership and emotional labor. Public Administration Review, 69(1), 6-20.

Ng, K. Y., \& Earley, P. C. (2006). Culture+ intelligence old constructs, new frontiers. Group \& Organization Management, 31(1), 4-19.

Norman-Major, K. (2011). Balancing the four Es: Or can we achieve equity for social equity in public administration? Journal of Public Affairs Education 17(2), 233252.

Norman-Major, K. (2012). Cultural competency across the master's in public administration curriculum. In K. Norman-Major \& S. Gooden (Eds.), Cultural competency for public administration (pp. 310-330). Armonk, NY: M.E. Sharpe.

Norman-Major, K. A., \& Gooden, S. T. (Eds.). (2012). Cultural competency for public administrators. Armonk, NY: M.E. Sharpe.

Nunnally, J. C., \& Bernstein, I. H. (1994). Psychometric theory (3rd ed.). New York: McGraw-Hill. 
O'Leary, R., Van Slyke, D. M., \& Kim, S. (Eds.). (2011). The future of public administration around the world: The Minnowbrook perspective. Washington, D.C.: Georgetown University Press.

Overeem, P. (2005). The value of the dichotomy: Politics, administration, and the political neutrality of administrators. Administrative Theory \& Praxis, 27(2), 311329.

Papadopoulos, I., Shea, S., Taylor, G., Pezzella, A., \& Foley, L. (2016). Developing tools to promote culturally competent compassion, courage, and intercultural communication in healthcare. Journal of Compassionate Health Care, 3(2).

Palomba, C., \& Banta, T. (1999). Assessment essentials: Planning, implementing, and improving assessment in higher education. San Francisco: Jossey-Bass.

Pandey, S. K., B. E. Wright, and D. P. Moynihan. (2008). Public service motivation and interorganizational citizenship behavior: Testing a preliminary model.

International Public Management Journal 11, 89-108.

Perry, J. L. (1996). Measuring public service motivation: An assessment of construct reliability and validity. Journal of Public Administration Research and Theory, $6(1), 5-22$.

Perry, J. L. (1997). Antecedents of public service motivation. Journal of Public Administration Research and Theory, 7(2), 181-197.

Perry, J. L., \& Wise, L. R. (1990). The motivational bases of public service. Public Administration Review, 50(3), 367-373.

Pike, G. R., \& Kuh, G. D. (2006). Relationships among structural diversity, informal peer interactions and perceptions of the campus environment. The Review of Higher Education, 29(4), 425-450.

Ping, R. A. (2009). "Is there any way to improve Average Variance Extracted (AVE) in a Latent Variable (LV) X (Revised)?" [on-line paper]. http://home.att.net/ rpingjr/ImprovAVE1.doc

Podsakoff, P. M., MacKenzie, S. B., \& Podsakoff, N. P. (2012). Sources of method bias in social science research and recommendations on how to control it. Annual review of psychology, 63, 539-569.

Price, E. G., Beach, M. C., Gary, T. L., Robinson, K. A., Gozu, A., Palacio, A., \& Powe, N. R. (2005). A systematic review of the methodological rigor of studies evaluating cultural competence training of health professionals. Academic Medicine, 80(6), 578-586. 
Reimann, J. O., Talavera, G. A., Salmon, M., Nuñez, J. A., \& Velasquez, R. J. (2004). Cultural competence among physicians treating Mexican Americans who have diabetes: a structural model. Social Science \& Medicine, 59(11), 2195-2205.

Reyes, H., Hadley, L., \& Davenport, D. (2013). A comparative analysis of cultural competence in beginning and graduating nursing students. ISRN Nursing, 2013, 15.

Rice, M \& Mathews, A. (2012). A new kind of public service professional: Possessing cultural competency awareness, knowledge, and skills. In K. Norman-Major \& S. Gooden (Eds.), Cultural competency for public administration (pp. 19-31). Armonk, NY: M.E. Sharpe.

Rice, M. F. (2007a). A post-modern cultural competency framework for public administration and public service delivery. International Journal of Public Sector Management, 20(7), 622-637.

Rice, M.F. (2007b). Promoting cultural competency in public administration and public service delivery: Utilizing self-assessment tools and performance measures. Journal of Public Affairs Education, 13(1), 41-57.

Rivera, M., Johnson, G., \& Kodaseet, G. (2012). A dialogic model for cultural competency in the graduate classroom. In K. Norman-Major \& S. Gooden (Eds.), Cultural competency for public administration (pp. 276-293). Armonk, NY: M.E. Sharpe.

Rowe, G. \& Wright, G. (1999). The Delphi method as a forecasting tool: Issues and analysis. International Journal of Forecasting, 15(4), 353 - 375.

Rubaii, N., \& Calarusse, C. (2012). Cultural Competency as a Standard for Accreditation. In Norman-Major, K. A., \& Gooden, S. T. (Eds.). Cultural competency for public Administrators (pp.219-243). ME Sharpe.

Rubaii, N., \& Calarusse, C. (2014). Preparing Public Service Professionals for a Diverse and Changing Workforce and Citizenry: Evaluating the Progress of NASPAA Programs in Competency Assessment. Journal of Public Affairs Education, 20(3), 285-304.

Ruddock, H. C., \& Turner, D. S. (2007). Developing cultural sensitivity: nursing students' experiences of a study abroad programme. Journal of Advanced Nursing, 59(4), 361-369.

Saha, S., Beach, M. C., \& Cooper, L. A. (2008). Patient centeredness, cultural competence and healthcare quality. Journal of the National Medical Association, 100(11), 1275. 
Saldivar, K. M. (2015). Team-Based Learning: A Model for Democratic and Culturally Competent 21st Century Public Administrators. Journal of Public Affairs Education, 21(2), 143-164.

Sargent, S. E., Sedlak, C. A., \& Martsolf, D. S. (2005). Cultural competence among nursing students and faculty. Nurse Education Today, 25(3), 214-221.

Schim, S. M., Doorenbos, A. Z., \& Borse, N. N. (2005). Cultural competence among Ontario and Michigan healthcare providers. Journal of Nursing Scholarship, 37(4), 354-360.

Schim, S. M., Doorenbos, A. Z., \& Borse, N. N. (2006). Cultural competence among hospice nurses. Journal of Hospice \& Palliative Nursing, 8(5), 302-307.

Schreiber, J. B., Nora, A., Stage, F. K., Barlow, E. A., \& King, J. (2006). Reporting structural equation modeling and confirmatory factor analysis results: A review. The Journal of Educational Research, 99(6), 323-338.

Skulmoski, G., Hartman, F., \& Krahn, J. (2007). The Delphi method for graduate research. Journal of Information Technology Education: Research, 6(1), 1-21.

Sodowsky, G. R., Kuo-Jackson, P. Y., Richardson, M. F., \& Corey, A. T. (1998). Correlates of self-reported multicultural competencies: Counselor multicultural social desirability, race, social inadequacy, locus of control racial ideology, and multicultural training. Journal of Counseling Psychology, 45, 256-264.

Sodowsky, G. R., Kuo-Jackson, P. Y., Richardson, M. F., \& Corey, A. T. (1998). Correlates of self-reported multicultural competencies: Counselor multicultural social desirability, race, social inadequacy, locus of control racial ideology, and multicultural training. Journal of Counseling Psychology, 45(3), 256-264.

Soper, D. (2017). Calculator: A-priori Sample Size for Multiple Regression. Retrieved January 28, 2017, from http://www.danielsoper.com/statcalc/calculator.aspx?id=1

Spanierman, L. B., Poteat, V. P., d, Y. F., \& Oh, E. (2008). Psychosocial costs of racism to white counselors: Predicting various dimensions of multicultural counseling competence. Journal of Counseling Psychology, 55(1), 75-88.

Standard 5 matching operations with the mission: Student learning. (2016). Retrieved from https://naspaaaccreditation.files.wordpress.com/2017/01/standard-5-textssi.pdf

Stemler, S. E. (2004). A comparison of consensus, consistency, and measurement approaches to estimating interrater reliability. Practical Assessment, Research \& Evaluation, 9(4), 1-19. 
Sue, D. (2001). Multidimensional facets of cultural competence. The Counseling Psychologist, 29(6), 790-821.

Tarca, K. (2005). Colorblind in control: The risks of resisting difference amid demographic change. Educational Studies, 38(2), 99-120.

Terenzini, P. T., Cabrera, A. F., Colbeck, C. L., Bjorklund, S. A., \& Parente, J. M. (2001). Racial and ethnic diversity in the classroom: Does it promote student learning? The Journal of Higher Education, 72(5), 509-531.

Tilford Group. (2001). Multicultural competency development: Preparing students to live and work in a diverse world. Retrieved April 15, 2015, from http://www.tilford.ksu.edu/p. aspx?tabid=32

U.S. Census Bureau Projections Show a Slower Growing, Older, More Diverse Nation a Half Century from Now. (2012, December 12). Retrieved January 28, 2017, from http://www.census.gov/newsroom/releases/archives/population/cb12-243.html

United States Census Bureau. (2014). 2014 National population projections: Summary tables. Retrieved from http://www.census.gov/population/projections/ data/national/2014/ summarytables.html

Vera, E. M., \& Speight, S. L. (2003). Multicultural competence, social justice, and counseling psychology: Expanding our roles. The Counseling Psychologist, 31(3), 253-272.

Wheaton, B., Muthen, B., Alwin, D. F., \& Summers, G. F. (1977). Assessing reliability and stability in panel models. Sociological Methodology, 8, 84-136.

White, R.L. (2008). The association of social responsibility endorsement with racerelated experiences, racial attitudes, and psychological outcomes among black college students. (Doctoral Dissertation). Retrieved from Deep Blue, University of Michigan.

White, S. (2004). Multicultural MPA curriculum: Are we preparing culturally competent public administrators? Journal of Public Affairs Education, 10(2), 111-123.

White, S. (2005). Social equity in the long haul: Preparing culturally competent public administrators. (Doctoral Dissertation). Retrieved from VTechWorks.

Worthington, R. L., Mobley, M., Franks, R. P., \& Tan, J. A. (2000). Multicultural counseling competencies: Verbal content, counselor attributions, and social desirability. Journal of Counseling Psychology, 47(4), 460-468.

Wright, B. E. (2007). Public service and motivation: Does mission matter? Public Administration Review, 67(1), 54-64. 
Wright, B. E., \& Grant, A. M. (2010). Unanswered questions about public service motivation: Designing research to address key issues of emergence and effects. Public Administration Review, 70(5), 691-700. 


\section{APPENDIX A}

\begin{tabular}{|c|c|c|c|c|}
\hline & Proficient -4 & Competent - 3 & Pre-Competent - 2 & Novice -1 \\
\hline Cultural Knowledge & $\begin{array}{l}\text { Student identifies that that there } \\
\text { is a specific cultural conflict in } \\
\text { the scenario. }\end{array}$ & $\begin{array}{l}\text { Student identifies the conflict as } \\
\text { related to a surface dimension of } \\
\text { culture (e.g. communication; } \\
\text { discomfort/tension between groups) } \\
\text { but does explicitly connect the } \\
\text { dimension to culture. }\end{array}$ & $\begin{array}{l}\text { Student is aware that there is a } \\
\text { conflict but does not specify the } \\
\text { type of conflict; or student } \\
\text { identifies conflict that is not } \\
\text { related to culture or a dimension of } \\
\text { culture. }\end{array}$ & $\begin{array}{l}\text { Unable to identify that a conflict } \\
\text { has occurred. }\end{array}$ \\
\hline Cultural Awareness & $\begin{array}{l}\text { Student can identify the } \\
\text { characteristics that contributed } \\
\text { to the conflict and demonstrates } \\
\text { cultural awareness through } \\
\text { recognition of cultural rules and } \\
\text { biases through explicit reference } \\
\text { to lack of awareness, prejudice, } \\
\text { stereotype, racism, assumption, } \\
\text { etc. }\end{array}$ & $\begin{array}{l}\text { Student can identify both individual } \\
\text { and organizational characteristics } \\
\text { (when applicable) that contributed to } \\
\text { the conflict; and begins to recognize } \\
\text { cultural rules and biases (e.g. } \\
\text { inadequate org. capacity; discomfort } \\
\text { or tension; lack of } \\
\text { openness/understanding; difference } \\
\text { in values/norms). }\end{array}$ & $\begin{array}{l}\text { Student does not identify all } \\
\text { relevant contributing factors. }\end{array}$ & $\begin{array}{l}\text { Does not identify any } \\
\text { contributing factors; or } \\
\text { demonstrates intolerance for one } \\
\text { of the presented views. }\end{array}$ \\
\hline Cultural Skills & $\begin{array}{l}\text { Applies cultural awareness and } \\
\text { knowledge to find a solution to } \\
\text { the cultural conflict through } 3 \\
\text { solutions (e.g. training, } \\
\text { assessment, resources). }\end{array}$ & $\begin{array}{l}\text { Applies cultural awareness and } \\
\text { knowledge to find a solution to the } \\
\text { cultural conflict through at least } 2 \\
\text { solutions. }\end{array}$ & $\begin{array}{l}\text { Applies cultural awareness and } \\
\text { knowledge to find at least one } \\
\text { solution to the cultural conflict. }\end{array}$ & $\begin{array}{l}\text { Is unable to develop a solution to } \\
\text { the cultural conflict. Identifies } \\
\text { inappropriate solution }\end{array}$ \\
\hline
\end{tabular}

Adapted from:

http://oregonstate.edu/studentaffairs/sites/default/files/docs/FinalVersionofInterculturalKnowledgeandEffectivenessRubric.pdf

https://www.aacu.org/value/rubrics/intercultural-knowledge 
VITA

\section{BRIDGETTE CRAM}

Born, Philadelphia, Pennsylvania

2006-2009

B.A., International Affairs and Spanish

Florida State University

Tallahassee, Florida

2010-2011

M.S., Higher Education

Florida State University

Tallahassee, Florida

2009-2011

Academic Program Officer

Florida State University

Tallahassee, Florida

2012-2015

Coordinator, Institutional Assessment

Florida International University

Miami, Florida

2015- Present

Student Success Manager

Florida International University

Miami, Florida

\section{SELECTED PUBLICATIONS AND PRESENTATIONS}

Cram, B. \& Alkadry, M. (2018). Social Equity Leadership. In A. Farazmand (Ed.) Global encyclopedia of public administration and public policy. Springer

Cram, B., \& Garcia-Zamor, J.C. (2015). Enhancing community and economic development post-disaster through the increased resilience of women. In D. Downey (Ed.) Cities and disasters. CRC Press.

Cram, B., Alkadry, M., \& Tower, L. (2015). The career-family tradeoff. In M. Connerly \& J. Wu (Eds.), handbook on well-being of working women. Springer.

Cram, B. (2014). Women in the face of disaster: Incorporating gender perspectives into disaster policy. Quick response grant report series; 247. 
Cram, B. (2014). Women in the face of disaster: Formulating gender-sensitive policy. PA Times.

Tandberg, D., Dorius, S. \& Cram, B. Ranking colleges and universities on returns to education: Institutional variation in expected returns on investment in higher education with a human capital framework. Educational policy analysis archives. (Revise and Resubmit).

Cram, B. \& Alkadry, M. Cultural competency in public administration education: A virtue ethics perspective. (In Progress)

Cram, B. \& Ganapati, E.N. Women in the face of disaster: A content analysis of vulnerability literature (In Progress)

Cram, B. (2016, October). Measuring and Predicting the Cultural Competency of MPA Students. Paper presented for presentation at the Network of Schools of Public Policy, Affairs, and Administration Annual Conference. Columbus, $\mathrm{OH}$.

Cram, B. \& Alkadry, M. (2016, March). Cultural Competence and Public Service. Paper presented at the America Society for Public Administration. Seattle, WA.

Cram, B. (2015, October). Cultural Competency Inclusion and Assessment within MPA Programs. Paper submitted for presentation at the Network of Schools of Public Policy, Affairs, and Administration Annual Conference. New York, NY.

Cram, B. \& Alkadry, M. (2015, October). Cultural Competence and Virtue Ethics Competence in Public Service Education. Paper submitted for presentation at the Network of Schools of Public Policy, Affairs, and Administration Annual Conference. New York, NY.

Cram, B. \& Alkadry, M. (2015, June). The Role of Cultural Competency Assessment in Achieving Social Equity. Paper to be presented at the Social Equity Leadership Conference. Nashville, TN.

Cram, B. (2015, March). Enhancing Public Service Delivery: Integrating Cultural Competence into the MPA Curriculum. Paper presented at the America Society for Public Administration. Washington, D.C.

Cram, B. (2014, September). An Ethical Argument for Enhancing Cultural Competence Curriculum in Public Administration. Paper presented at the Southeastern Conference for Public Administration.

Cram, B. \& Alkadry, M. (2014, May). Postcolonial revisiting of Public Administration Theory. Paper presented at the Public Administration Theory Network Conference, Miami, FL. 